\title{
WestVirginiaUniversity
}

THE RESEARCH REPOSITORY @ WVU

Graduate Theses, Dissertations, and Problem Reports

2000

\section{Fine-scale modeling of failure in an adhesive layer}

Pulkit V. D. Mathur

West Virginia University

Follow this and additional works at: https://researchrepository.wvu.edu/etd

\section{Recommended Citation}

Mathur, Pulkit V. D., "Fine-scale modeling of failure in an adhesive layer" (2000). Graduate Theses, Dissertations, and Problem Reports. 1005.

https://researchrepository.wvu.edu/etd/1005

This Thesis is protected by copyright and/or related rights. It has been brought to you by the The Research Repository @ WVU with permission from the rights-holder(s). You are free to use this Thesis in any way that is permitted by the copyright and related rights legislation that applies to your use. For other uses you must obtain permission from the rights-holder(s) directly, unless additional rights are indicated by a Creative Commons license in the record and/ or on the work itself. This Thesis has been accepted for inclusion in WVU Graduate Theses, Dissertations, and Problem Reports collection by an authorized administrator of The Research Repository @ WVU. For more information, please contact researchrepository@mail.wvu.edu. 


\title{
FINE-SCALE MODELING OF FAILURE IN AN ADHESIVE LAYER
}

\author{
Pulkit V. D. Mathur \\ Thesis submitted to the \\ College of Arts and Sciences \\ at West Virginia University \\ in partial fulfillment of the requirements \\ for the degree of
}
Master of Science
in
Physics

M. Ferer, Ph.D., Chair

D. H. Smith, Ph.D.

L. Halliburton, Ph.D.

Department of Physics

Morgantown, West Virginia 2000

Keywords: Material Failure, Cohesive Forces, Filter Cake, Quasi-Static Copyright 2000 Pulkit Mathur 


\section{Abstract \\ Fine-Scale Modeling of Failure in an Adhesive Layer \\ By Pulkit Mathur}

Rigid ceramic filter media are used for removing particulate matter from gas streams at high temperatures. As the particulate matter (dust cake) collects on the surface of the filter, the filtration pressure increases; to maintain an economical operation the dust cake must be removed periodically by back-pulse cleaning. Often the dust cake is not removed completely by the back-pulse pressure and the resulting patchy cleaning affects operation.

The thesis presents the results from a fine scale modeling of the removal of a layer of filter cake by a time dependent pressure pulse. In this model the filter cake is grid in 16,000 imaginary blocks on a filter, of area $160 \mathrm{~cm}^{2}$. The cleaning of the filter is simulated through 10 consecutive cleaning cycles. The model includes both adhesive forces between the blocks of the filter cake and the filter, as well as cohesive forces between neighboring blocks of filter cake.

The evolution of the cleaning from one cycle to the next is studied. The results show that for each cycle, cohesive forces lower the pressures required for cleaning because they increase the stress near broken adhesive bonds. They also cause thicker cakes to be removed more efficiently. An interesting observation is that there are regions where the filter cake is lifted up but not removed during one cycle; many of these regions are removed during the next cycle. The model enables a detailed quantitative investigation of way this effect depends on the filter cake thickness and the strength of the applied pressure pulse. 
To my parents 


\section{Acknowledgments}

I would like to express my sincere gratitude to my advisor Dr. Ferer for his patience, guidance and encouragement which made this work possible. I deeply appreciate his always being there to answer all my questions. I am very grateful to my committee members, Dr. D. H. Smith and Dr. Larry Halliburton for their time, support and guidance. I thank the U. S. Department of Energy for funding this project.

I remain deeply indebted to all my professors in the Department of Physics, and Dr. Ian Christie in the Department of Mathematics, at West Virginia University, for helping me explore my own potentials and for making my two years at West Virginia University very happy and memorable. I also take this opportunity to express my deep gratitude to Jim and Joey Colbert, for providing me with a second home and a loving and caring family, in the United States. 


\section{Table of Contents}

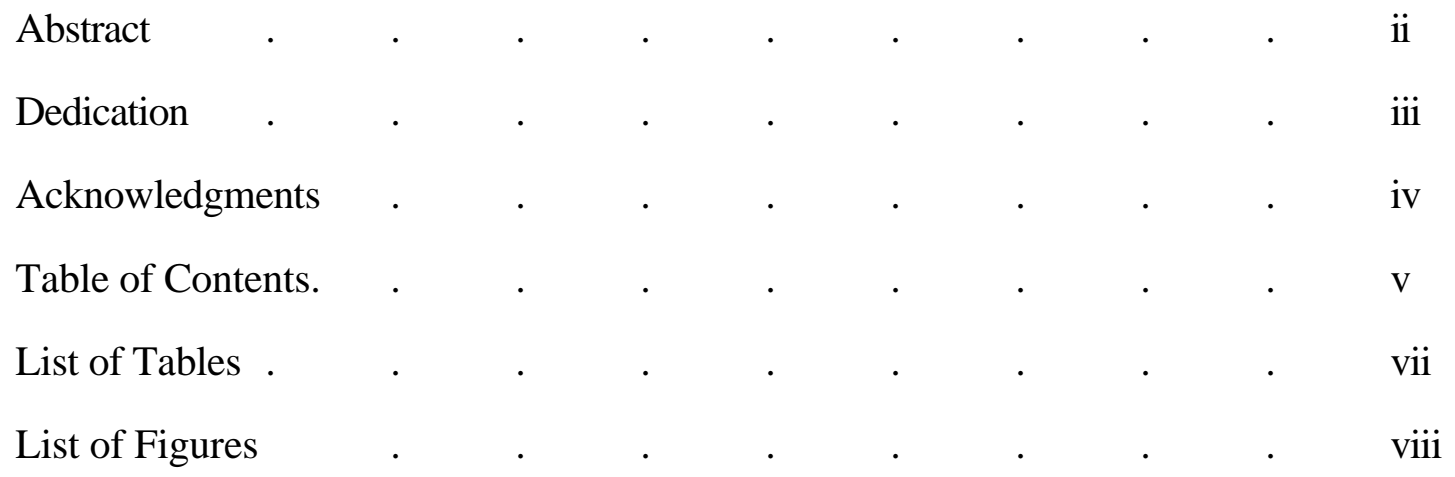

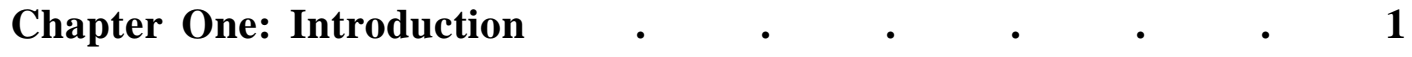

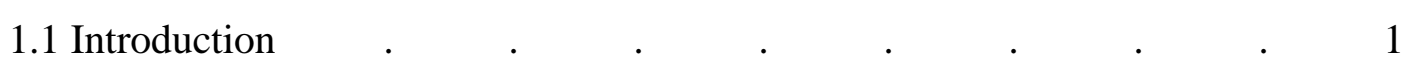

Chapter Two: Fine-Scale Filter Cake Model • $\quad$ • $\quad$. $\quad 3$

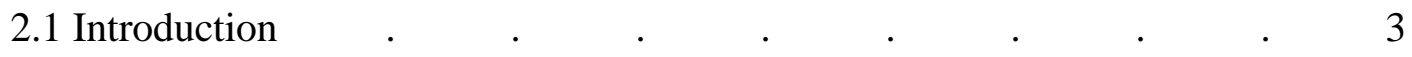

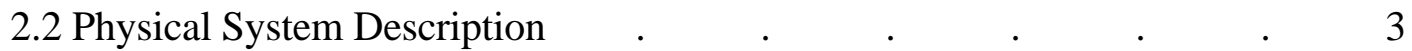

2.3 Description of Fine Scale Filter Cake Model $\quad$. $\quad$. $\quad$. $\quad$. 3

$\begin{array}{llllllllllll}\text { 2.4 Filter Cake removal as a Quasi-Static Process } & \text {. } & \text {. } & & & & & & & \end{array}$

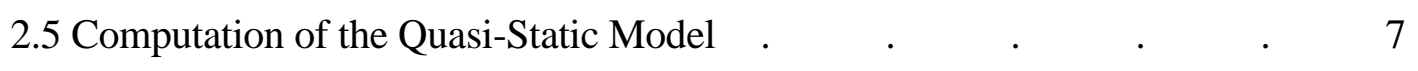

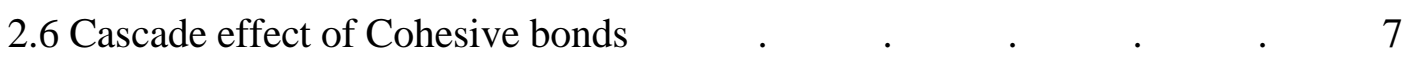

Chapter Three: Efficiency of Filter Cake Removal . $\quad$. $\quad 9$

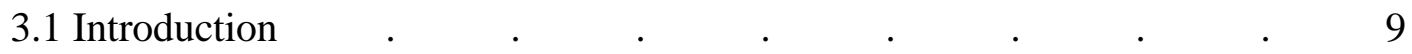

3.2 Layer removal as a function of time $\quad . \quad$. $\quad . \quad$. $\quad$. $\quad 10$

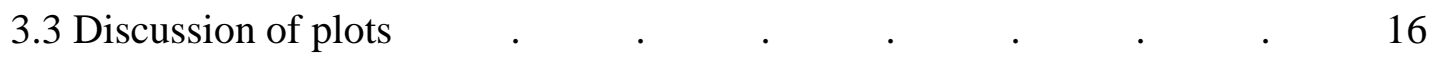

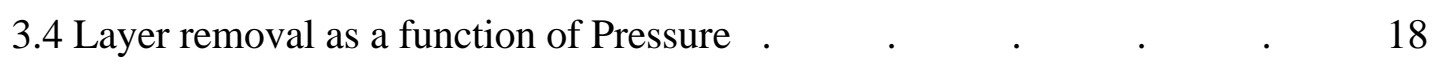

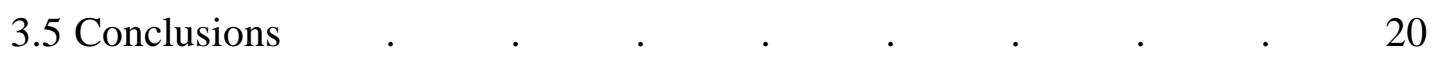




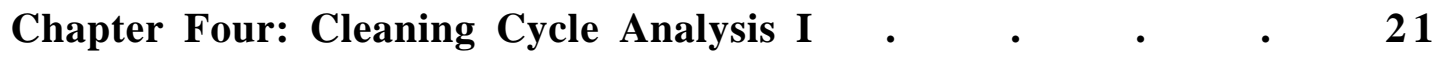

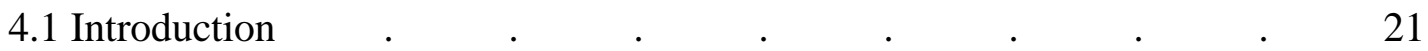

4.2 Blocks which are removed in two consecutive cycles $\quad$. $\quad$. $\quad 21$

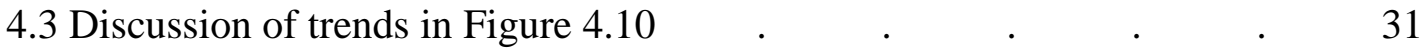

4.4 Blocks which are lifted in one cycle and removed in the next $\quad$. $\quad$. $\quad 32$

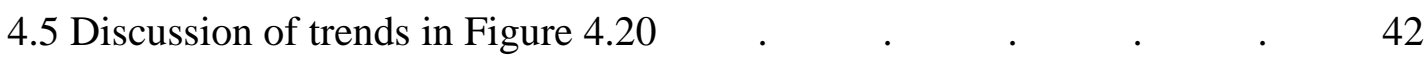

4.6 Conclusions from Figure 4.10 and 4.20 . $\quad . \quad$. $\quad . \quad$. $\quad . \quad 43$

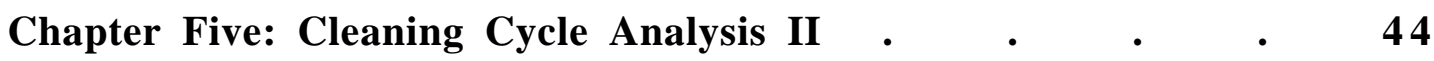

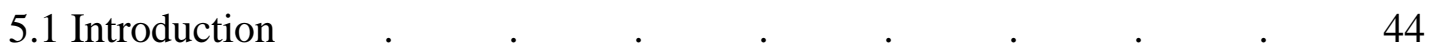

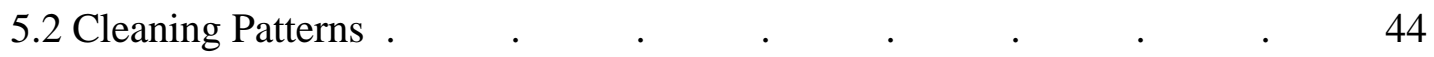

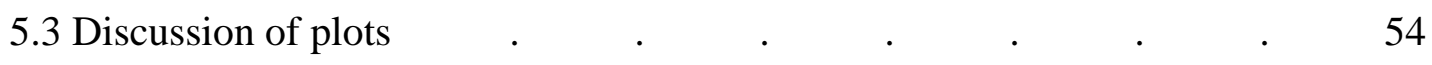

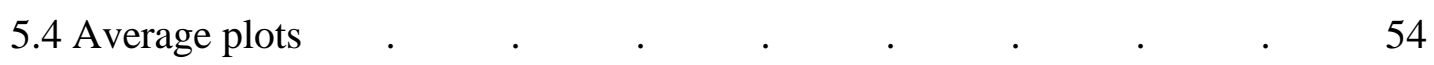

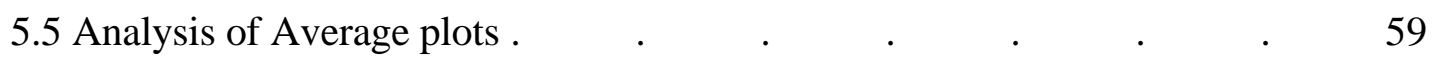

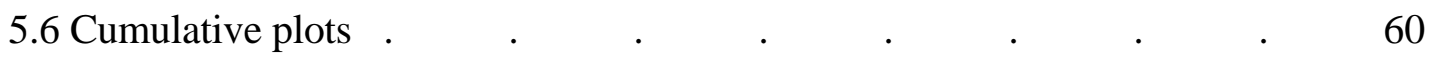

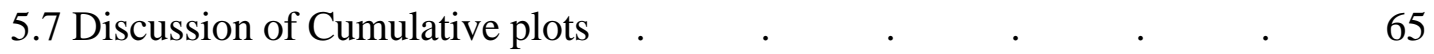

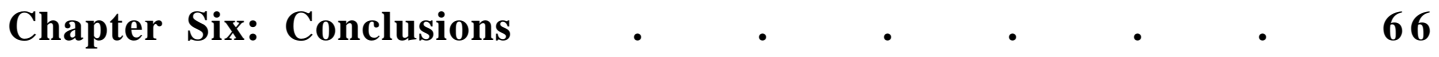

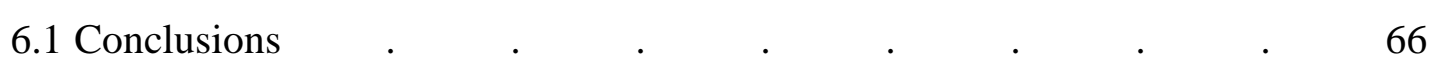

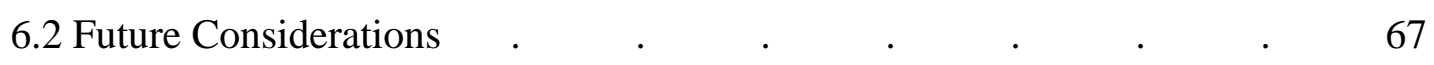

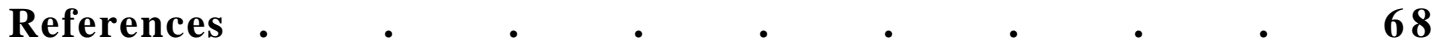

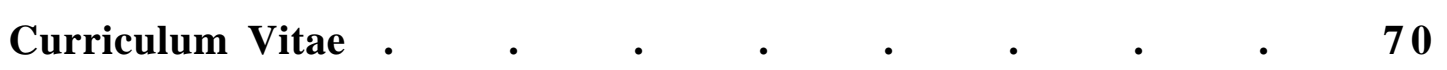




\section{List of Tables}

Table 3.1 Thickness parameters and their corresponding midpoint pressures $\ldots 11$

Table 3.2a Differential pressures and the corresponding limiting values of $\ldots 17$ fraction of mass removed, for thickness parameter $\mathrm{T}=0.5$.

Table 3.2b Differential pressures and the corresponding limiting values of $\ldots 18$ fraction of mass removed, for thickness parameter $\mathrm{T}=1.0$.

Table 3.2c Differential pressures and the corresponding limiting values of $\ldots 18$ fraction of mass removed, for thickness parameter $\mathrm{T}=1.5$. 


\section{List of Figures}

Figure 2.1 Fine-Scale Filter Cake model $\ldots 4$

Figure 3.1a Plot of Fraction of mass removed Vs. Time step, for thickness . . 12 parameter $\mathrm{T}=0.5$ and pressure $\mathrm{P}(\mathrm{t})=\left(P_{\frac{1}{2}}+0.04\right)(1-\mathrm{f})$ for all 10 cleaning cycles

Figure 3.1b Plot of Fraction of mass removed Vs. Time step, for thickness . . 12 parameter $\mathrm{T}=0.5$ and pressure $\mathrm{P}(\mathrm{t})=\left(P_{\frac{1}{2}}+0.01\right)(1-\mathrm{f})$ for all 10 cleaning cycles

Figure 3.1c Plot of Fraction of mass removed Vs. Time step, for thickness . . 13 parameter $\mathrm{T}=0.5$ and pressure $\mathrm{P}(\mathrm{t})=\left(P_{\frac{1}{2}}-0.02\right)(1-\mathrm{f})$ for all 10 cleaning cycles

Figure 3.2a Plot of Fraction of mass removed Vs. Time step, for thickness . . 13 parameter $\mathrm{T}=1.0$ and pressure $\mathrm{P}(\mathrm{t})=\left(P_{\frac{1}{2}}+0.04\right)(1-\mathrm{f})$ for all 10 cleaning cycles

Figure 3.2b Plot of Fraction of mass removed Vs. Time step, for thickness . . 14 parameter $\mathrm{T}=1.0$ and pressure $\mathrm{P}(\mathrm{t})=\left(P_{\frac{1}{2}}+0.02\right)(1-\mathrm{f})$ for all 10 cleaning cycles

Figure 3.2c Plot of Fraction of mass removed Vs. Time step, for thickness . . 14 parameter $\mathrm{T}=1.0$ and pressure $\mathrm{P}(\mathrm{t})=\left(P_{\frac{1}{2}}-0.01\right)(1-\mathrm{f})$ for all 10 cleaning cycles 
Figure 3.3a Plot of Fraction of mass removed Vs. Time step, for thickness . . 15 parameter $\mathrm{T}=1.5$ and pressure $\mathrm{P}(\mathrm{t})=\left(P_{\frac{1}{2}}+0.04\right)(1-\mathrm{f})$ for all 10 cleaning cycles

Figure 3.3b Plot of Fraction of mass removed Vs. Time step, for thickness . . 15 parameter $\mathrm{T}=1.5$ and pressure $\mathrm{P}(\mathrm{t})=\left(P_{\frac{1}{2}}+0.02\right)(1-\mathrm{f})$ for all 10 cleaning cycles

Figure 3.3c Plot of Fraction of mass removed Vs. Time step, for thickness . . 16 parameter $\mathrm{T}=1.5$ and pressure $\mathrm{P}(\mathrm{t})=\left(P_{\frac{1}{2}}+0.0\right)(1-\mathrm{f})$ for all 10 cleaning cycles

Figure 3.4 Plot of Fraction of mass removed Vs. Maximum applied Pressure _. 19 $P_{\max }=P_{\frac{1}{2}}+\mathrm{dP}$, for the 3 thickness parameters under consideration

Figure 4.1a Plot of the total number of blocks removed in cycle $n$ Vs. the $\ldots 22$ cycle number $\mathrm{n}$, for thickness parameter $\mathrm{T}=0.5$ and maximum pressure $P_{\max }=P_{\frac{1}{2}}+0.02$ for each cycle

Figure 4.1b Plot of Fraction of those blocks removed in cycle n, which are .. 22 also removed in cycle $n+1$ Vs. cycle number $n$, for $T=0.5$ and $P_{\max }=P_{\frac{1}{2}}+0.02$ for each cycle

Figure 4.2a Plot of the total number of blocks removed in cycle $n$ Vs. the $\ldots 23$ cycle number $\mathrm{n}$, for thickness parameter $\mathrm{T}=0.5$ and maximum of pressure $P_{\max }=P_{\frac{1}{2}}-0.02$ for each cycle 
Figure 4.2b Plot of Fraction of those blocks removed in cycle $n$, which are $\ldots 23$ also removed in cycle $n+1$ Vs. cycle number $n$, for $T=0.5$ and $P_{\max }=P_{\frac{1}{2}}-0.02$ for each cycle

Figure 4.3a Plot of the total number of blocks removed in cycle $n$ Vs. the .. 24 cycle number $\mathrm{n}$, for thickness parameter $\mathrm{T}=0.5$ and maximum pressure $P_{\max }=P_{\frac{1}{2}}-0.04$ for each cycle

Figure 4.3b Plot of Fraction of those blocks removed in cycle $n$, which are $\ldots 24$ also removed in cycle $n+1$ Vs. cycle number $n$. for $T=0.5$ and $P_{\max }=P_{\frac{1}{2}}-0.04$ for each cycle

Figure 4.4a Plot of the total number of blocks removed in cycle $n \mathrm{Vs}$. the $\ldots 25$ cycle number $\mathrm{n}$, for thickness parameter $\mathrm{T}=1.0$ and maximum pressure $P_{\max }=P_{\frac{1}{2}}+0.02$ for each cycle

Figure 4.4b Plot of Fraction of those blocks removed in cycle $n$, which are $\ldots 25$ also removed in cycle $n+1$ Vs. cycle number $n$, for $T=1.0$ and $P_{\max }=P_{\frac{1}{2}}+0.02$ for each cycle

Figure 4.5a Plot of the total number of blocks removed in cycle $n$ Vs. the . . 26 cycle number $\mathrm{n}$, for thickness parameter $\mathrm{T}=1.0$ and maximum pressure $P_{\max }=P_{\frac{1}{2}}+0.01$ for each cycle

Figure 4.5b Plot of Fraction of those blocks removed in cycle $n$, which are $\ldots 26$ also removed in cycle $n+1$ Vs. cycle number $n$, for $T=1.0$ and $P_{\max }=P_{\frac{1}{2}}+0.01$ for each cycle 
Figure 4.6a Plot of the total number of blocks removed in cycle $n$ Vs. the $\ldots 27$ cycle number $\mathrm{n}$, for thickness parameter $\mathrm{T}=1.0$ and maximum pressure $P_{\max }=P_{\frac{1}{2}}-0.01$ for each cycle

Figure 4.6b Plot of Fraction of those blocks removed in cycle n, which are . . 27 also removed in cycle $n+1$ Vs. cycle number $n$, for $T=1.0$ and $P_{\max }=P_{\frac{1}{2}}-0.01$ for each cycle

Figure 4.7a Plot of the total number of blocks removed in cycle $n$ Vs. the .. 28 cycle number $\mathrm{n}$, for thickness parameter $\mathrm{T}=1.5$ and maximum pressure $P_{\max }=P_{\frac{1}{2}}+0.03$ for each cycle

Figure 4.7b Plot of Fraction of those blocks removed in cycle n, which are . . 28 also removed in cycle $n+1$ Vs. cycle number $n$, for $T=1.5$ and $P_{\max }=P_{\frac{1}{2}}+0.03$ for each cycle

Figure 4.8a Plot of the total number of blocks removed in cycle $n$ Vs. the . . 29 cycle number $\mathrm{n}$, for thickness parameter $\mathrm{T}=1.5$ and maximum pressure $P_{\max }=P_{\frac{1}{2}}+0.02$ for each cycle

Figure 4.8b Plot of Fraction of those blocks removed in cycle n, which are . . 29 also removed in cycle $n+1$ Vs. cycle number $n$, for $T=1.5$ and $P_{\max }=P_{\frac{1}{2}}+0.02$ for each cycle

Figure 4.9a Plot of the total number of blocks removed in cycle $n$ Vs. the . . 30 cycle number $\mathrm{n}$, for thickness parameter $\mathrm{T}=1.5$ and maximum pressure $P_{\max }=P_{\frac{1}{2}}+0.0$ for each cycle 
Figure 4.9b Plot of Fraction of those blocks removed in cycle n, which are . . 30 also removed in cycle $n+1$ Vs. cycle number $n$, for $T=1.5$ and $P_{\max }=P_{\frac{1}{2}}+0.0$ for each cycle

Figure 4.10 A plot summarizing Figures $4.1 \mathrm{~b}-4.9 \mathrm{~b}$, showing trends in the $\ldots 31$ fraction of blocks that are removed in two successive cycles, as an average over the last few cycles Vs. differential pressure $\mathrm{dP}$, for the three thickness parameters $\mathrm{T}$

Figure 4.11a Plot of total number of blocks lifted in cycle $n$ Vs. the cycle .. 33 number $\mathrm{n}$, for thickness parameter $\mathrm{T}=0.5$ and maximum pressure $P_{\max }=P_{\frac{1}{2}}+0.02$ for each cycle

Figure 4.11b Plot of the number of blocks lifted in cycle $n$ and removed in cycle $\ldots 33$ $\mathrm{n}+1$, (as a fraction of the total number of blocks removed in cycle $\mathrm{n}+1)$ Vs. the cycle number $\mathrm{n}$, for thickness parameter $\mathrm{T}=0.5$ and maximum pressure $P_{\max }=P_{\frac{1}{2}}+0.02$ for each cycle

Figure 4.12a Plot of total number of blocks lifted in cycle $n$ Vs. the cycle ...34 number $\mathrm{n}$, for thickness parameter $\mathrm{T}=0.5$ and maximum pressure $P_{\max }=P_{\frac{1}{2}}-0.02$ for each cycle

Figure 4.12b Plot of the number of blocks lifted in cycle $n$ and removed in cycle $\ldots 34$ $\mathrm{n}+1$, (as a fraction of the total number of blocks removed in cycle $\mathrm{n}+1)$ Vs. the cycle number $\mathrm{n}$, for thickness parameter $\mathrm{T}=0.5$ and maximum pressure $P_{\max }=P_{\frac{1}{2}}-0.02$ for each cycle 
Figure 4.13a Plot of total number of blocks lifted in cycle $n$ Vs. the cycle . . 35 number $\mathrm{n}$, for thickness parameter $\mathrm{T}=0.5$ and maximum pressure $P_{\max }=P_{\frac{1}{2}}-0.04$ for each cycle

Figure 4.13b Plot of the number of blocks lifted in cycle $\mathrm{n}$ and removed in cycle $\ldots 35$ $\mathrm{n}+1$, (as a fraction of the total number of blocks removed in cycle $n+1)$ Vs. the cycle number $n$, for thickness parameter $T=0.5$ and maximum pressure $P_{\max }=P_{\frac{1}{2}}-0.04$ for each cycle

Figure 4.14a Plot of total number of blocks lifted in cycle $n$ Vs. the cycle .. 36 number $\mathrm{n}$, for thickness parameter $\mathrm{T}=1.0$ and maximum pressure $P_{\max }=P_{\frac{1}{2}}+0.02$ for each cycle

Figure 4.14b Plot of the number of blocks lifted in cycle $n$ and removed in cycle $\ldots 36$ $\mathrm{n}+1$, (as a fraction of the total number of blocks removed in cycle $\mathrm{n}+1)$ Vs. the cycle number $\mathrm{n}$, for thickness parameter $\mathrm{T}=1.0$ and maximum pressure $P_{\max }=P_{\frac{1}{2}}+0.02$ for each cycle

Figure 4.15a Plot of total number of blocks lifted in cycle $n$ Vs. the cycle . . 37 number $\mathrm{n}$, for thickness parameter $\mathrm{T}=1.0$ and maximum pressure $P_{\max }=P_{\frac{1}{2}}+0.01$ for each cycle

Figure 4.15b Plot of the number of blocks lifted in cycle $\mathrm{n}$ and removed in cycle $\ldots 37$ $\mathrm{n}+1$, (as a fraction of the total number of blocks removed in cycle $\mathrm{n}+1)$ Vs. the cycle number $\mathrm{n}$, for thickness parameter $\mathrm{T}=1.0$ and maximum pressure $P_{\max }=P_{\frac{1}{2}}+0.01$ for each cycle 
Figure 4.16a Plot of total number of blocks lifted in cycle $n$ Vs. the cycle ...38 number $\mathrm{n}$, for thickness parameter $\mathrm{T}=1.0$ and maximum pressure $P_{\max }=P_{\frac{1}{2}}-0.01$ for each cycle

Figure 4.16b Plot of the number of blocks lifted in cycle $n$ and removed in cycle $\ldots 38$ $\mathrm{n}+1$, (as a fraction of the total number of blocks removed in cycle $\mathrm{n}+1)$ Vs. the cycle number $\mathrm{n}$, for thickness parameter $\mathrm{T}=1.0$ and maximum pressure $P_{\max }=P_{\frac{1}{2}}-0.01$ for each cycle

Figure 4.17a Plot of total number of blocks lifted in cycle n Vs. the cycle ...39 number $\mathrm{n}$, for thickness parameter $\mathrm{T}=1.5$ and maximum pressure $P_{\max }=P_{\frac{1}{2}}+0.03$ for each cycle

Figure 4.17b Plot of the number of blocks lifted in cycle $\mathrm{n}$ and removed in cycle $\ldots 39$ $\mathrm{n}+1$, (as a fraction of the total number of blocks removed in cycle $\mathrm{n}+1) \mathrm{Vs}$. the cycle number $\mathrm{n}$, for thickness parameter $\mathrm{T}=1.5$ and maximum pressure $P_{\max }=P_{\frac{1}{2}}+0.03$ for each cycle

Figure 4.18a Plot of total number of blocks lifted in cycle $n$ Vs. the cycle . . 40 number $\mathrm{n}$, for thickness parameter $\mathrm{T}=1.5$ and maximum pressure $P_{\max }=P_{\frac{1}{2}}+0.02$ for each cycle

Figure 4.18b Plot of the number of blocks lifted in cycle $n$ and removed in cycle $\ldots 40$ $\mathrm{n}+1$, (as a fraction of the total number of blocks removed in cycle $n+1)$ Vs. the cycle number $n$, for thickness parameter $T=1.5$ and maximum pressure $P_{\max }=P_{\frac{1}{2}}+0.02$ for each cycle 
Figure 4.19a Plot of total number of blocks lifted in cycle $n$ Vs. the cycle ..41 number $\mathrm{n}$, for thickness parameter $\mathrm{T}=1.5$ and maximum pressure $P_{\max }=P_{\frac{1}{2}}+0.0$ for each cycle

Figure 4.19b Plot of the number of blocks lifted in cycle $\mathrm{n}$ and removed in cycle $\ldots 41$ $\mathrm{n}+1$, (as a fraction of the total number of blocks removed in cycle $\mathrm{n}+1)$ Vs. the cycle number $\mathrm{n}$, for thickness parameter $\mathrm{T}=1.5$ and maximum pressure $P_{\max }=P_{\frac{1}{2}}+0.0$ for each cycle

Figure 4.20 A summarized plot of Figures 4.11b-4.19b, showing trends in the $\ldots 42$ fraction of blocks that are lifted in one cycle and removed in the next cycle, (average over the last few cycles) Vs. differential pressure $\mathrm{dP}$, for the three thickness parameters $\mathrm{T}$

Figure 5.1a Plot of the filter cake layer for thickness parameter $\mathrm{T}=0.5$ and $\ldots 45$ maximum pressure $P_{\max }=P_{\frac{1}{2}}+0.02$

Figure 5.1b Plot of the filter cake layer for thickness parameter $\mathrm{T}=0.5$ and $\ldots 46$ maximum pressure $P_{\max }=P_{\frac{1}{2}}-0.02$

Figure 5.1c Plot of the filter cake layer for thickness parameter $\mathrm{T}=0.5$ and $\ldots 47$ maximum pressure $P_{\max }=P_{\frac{1}{2}}-0.04$

Figure 5.2a Plot of the filter cake layer for thickness parameter $\mathrm{T}=1.0$ and $\ldots 48$ maximum pressure $P_{\max }=P_{\frac{1}{2}}+0.02$

Figure 5.2b Plot of the filter cake layer for thickness parameter $\mathrm{T}=1.0$ and $\ldots 49$ maximum pressure $P_{\max }=P_{\frac{1}{2}}+0.01$ 
Figure 5.2c Plot of the filter cake layer for thickness parameter $\mathrm{T}=1.0$ and $\ldots 50$ maximum pressure $P_{\max }=P_{\frac{1}{2}}-0.01$

Figure 5.3a Plot of the filter cake layer for thickness parameter $\mathrm{T}=1.5$ and $\ldots 51$ maximum pressure $P_{\max }=P_{\frac{1}{2}}+0.03$

Figure 5.3b Plot of the filter cake layer for thickness parameter $\mathrm{T}=1.5$ and $\ldots 52$ maximum pressure $P_{\max }=P_{\frac{1}{2}}+0.02$

Figure 5.3c Plot of the filter cake layer for thickness parameter $\mathrm{T}=1.5$ and $\ldots 53$ maximum pressure $P_{\max }=P_{\frac{1}{2}}+0.0$

Figure 5.4a Plot of total number of blocks removed Vs. the frequency . . 55 (number of cycles) with which they were removed, for $\mathrm{T}=0.5$ and maximum pressure $P_{\max }=P_{\frac{1}{2}}+0.02$

Figure 5.4b Plot of total number of blocks removed Vs. the frequency . . 55 (number of cycles) with which they were removed, for $\mathrm{T}=0.5$ and maximum pressure $P_{\max }=P_{\frac{1}{2}}-0.02$

Figure 5.4c Plot of total number of blocks removed Vs. the frequency . . 56 (number of cycles) with which they were removed, for $\mathrm{T}=0.5$ and maximum pressure $P_{\max }=P_{\frac{1}{2}}-0.04$

Figure 5.5a Plot of total number of blocks removed Vs. the frequency . . 56 (number of cycles) with which they were removed, for $\mathrm{T}=1.0$ and maximum pressure $P_{\max }=P_{\frac{1}{2}}+0.02$ 
Figure 5.5b Plot of total number of blocks removed Vs. the frequency . . 57 (number of cycles) with which they were removed, for $\mathrm{T}=1.0$ and maximum pressure $P_{\max }=P_{\frac{1}{2}}+0.01$

Figure 5.5c Plot of total number of blocks removed Vs. the frequency . . 57 (number of cycles) with which they were removed, for $\mathrm{T}=1.0$ and maximum pressure $P_{\max }=P_{\frac{1}{2}}-0.01$

Figure 5.6a Plot of total number of blocks removed Vs. the frequency ...58 (number of cycles) with which they were removed, for $\mathrm{T}=1.5$ and maximum pressure $P_{\max }=P_{\frac{1}{2}}+0.03$

Figure 5.6b Plot of total number of blocks removed Vs. the frequency ...58 (number of cycles) with which they were removed, for $\mathrm{T}=1.5$ and maximum pressure $P_{\max }=P_{\frac{1}{2}}+0.02$

Figure 5.6c Plot of total number of blocks removed Vs. the frequency ...59 (number of cycles) with which they were removed, for $\mathrm{T}=1.5$ and maximum pressure $P_{\max }=P_{\frac{1}{2}}+0.0$

Figure 5.7a Plot of the percentage of Cumulative area removed Vs. the ..60 frequency (number of cycles) of removal, for $\mathrm{T}=0.5$ and maximum pressure $P_{\max }=P_{\frac{1}{2}}+0.02$

Figure 5.7b Plot of the percentage of Cumulative area removed Vs. the ..61 frequency (number of cycles) of removal, for $\mathrm{T}=0.5$ and maximum pressure $P_{\max }=P_{\frac{1}{2}}-0.02$ 
Figure 5.7c Plot of the percentage of Cumulative area removed Vs. the ..61 frequency (number of cycles) of removal, for $\mathrm{T}=0.5$ and maximum pressure $P_{\max }=P_{\frac{1}{2}}-0.04$

Figure 5.8a Plot of the percentage of Cumulative area removed Vs. the ..662 frequency (number of cycles) of removal, for $\mathrm{T}=1.0$ and maximum pressure $P_{\max }=P_{\frac{1}{2}}+0.02$

Figure 5.8b Plot of the percentage of Cumulative area removed Vs. the . .62 frequency (number of cycles) of removal, for $\mathrm{T}=1.0$ and maximum pressure $P_{\max }=P_{\frac{1}{2}}+0.01$

Figure 5.8c Plot of the percentage of Cumulative area removed Vs. the ..63 frequency (number of cycles) of removal, for $\mathrm{T}=1.0$ and maximum pressure $P_{\max }=P_{\frac{1}{2}}-0.01$

Figure 5.9a Plot of the percentage of Cumulative area removed Vs. the ..63 frequency (number of cycles) of removal, for $\mathrm{T}=1.5$ and maximum pressure $P_{\max }=P_{\frac{1}{2}}+0.03$

Figure 5.9b Plot of the percentage of Cumulative area removed Vs. the . .64 frequency (number of cycles) of removal, for $\mathrm{T}=1.5$ and maximum pressure $P_{\max }=P_{\frac{1}{2}}+0.02$

Figure 5.9c Plot of the percentage of Cumulative area removed Vs. the ..664 frequency (number of cycles) of removal, for $\mathrm{T}=1.5$ and maximum pressure $P_{\max }=P_{\frac{1}{2}}+0.0$ 


\section{Chapter One \\ Introduction}

\subsection{Introduction}

Material failure is an issue of major importance and has, therefore been widely studied for well over a century. Much of this work has naturally focused on the build up of stress at defects, formation of cracks, energy changes during crack formation and dynamics of crack propagation $[5,6,8]$. A number of quasimicroscopic and microscopic models have been used to study these important questions [6-8]. The Fine-Scale filter cake model is similar in spirit to many of these quasimicroscopic models. It was developed with a view of addressing some of the problems encountered in the removal of a filter cake layer from cylindrical filters, during the backpulse cleaning cycle of filtration of hot gases in Pressurized Fluidized Bed Combustion (PFBC) Systems [1-4].

Some of the particle filter systems used in the PFBC's were seen to have a failure rate which exceeded acceptable levels required to maintain an economically viable operation. Failure of these filter systems was found to correlate with material properties of the filter cake removed [9]. Also since the filtration of gases was carried out at high temperatures, cohesive effects in the dust layer assumed importance. The fine-scale model's strengths lie in its ability to include both cohesive and adhesive forces, realistic strength distributions as well as variations in back pulse pressure, in a numerically reliable simulation. This would enable the design of a more efficient cleaning process and would remedy some of the difficulties encountered during the operation of the PFBC units.

Experimental studies of the cleaning of filters by backpulses of compressed gas, determined the following qualitative aspects of the cleaning process, 
1) The backpulse pressure applied from inside the candle filter, is essentially constant along the length of the filter $[10,11]$.

2) Cleaning efficiency increases with increase in filter cake thickness $[12,13]$.

3) Cleaning efficiency also increases with increase in applied pressure above a certain threshold pressure [11-13, 17, 18].

4) The filter cake is removed in larger (cm size) flakes, for larger thickness [11].

In addition to the above, the effects of dust properties [14] and temperature [15] on the strength and adhesion of the filter cake were also studied experimentally.

The fine-scale numerical modeling of the cleaning process, observed all of the above aspects. An increase in cleaning efficiency was observed with an increase in the strength of cohesive forces (i.e. increase in the filter cake thickness) $[1,2,4]$. The simulations studied the size and shape of filter cake fragments, and observed that larger fragments occurred for thicker more cohesive cakes [3]. In addition the modeled results of filter cleaning efficiency are seen to match closely with experimental results from literature [16].

In this thesis the Fine-Scale Filter Cake model has been used to study of the cleaning of a cylindrical filter surface (through 10 consecutive cleaning cycles) by a time dependent pressure pulse. The cleaning process is studied for three filter cakes, which differ from each other in the relative strengths of their cohesive and adhesive forces. The timeline of the thesis is as follows: Chapter Two describes the Fine-Scale filter cake model. Chapter Three studies the effect of cohesive forces and applied pressure on the cleaning efficiency of the filter. Lastly Chapters Four and Five analyze the trends in filter cake removal as the cleaning proceeds from one cycle to the next. 


\section{Chapter Two \\ Fine-Scale Filter Cake Model}

\subsection{Introduction}

The fine-scale model simulates the removal of a filter cake layer from the surface of a cylindrical filter, by a force applied perpendicular to the layer. The model is motivated by problems encountered in the removal of the filter cake layer from cylindrical filters during the back-pulse cleaning cycle of filtration of hot gases from Pressurized Fluidized Bed Combustion (PFBC) systems [1]. A discussion of the model is presented in this chapter. The fine scale-model includes both the adhesive forces (which try to maintain contact between the filter cake layer and the substrate) and the cohesive forces (which maintain integrity of the deposited layer). It separates these two effects thus enabling an independent study of various features of material failure [1-3].

\subsection{Physical System Description}

The physical system comprises a cylindrical candle filter as shown in Figure 2.1a, on which a layer of filter cake of some thickness $t$ is deposited. The filter is cleaned by applying a back-pulse of compressed air from the inside of the candle filter, thus blowing off the deposited layer. The pressure actually responsible for removing the filter cake is the pressure drop P across the layer [1-3].

\subsection{Description of the Fine-Scale, Filter Cake model}

In this model, the filter cake is gridded into rectangular blocks of thickness $\mathrm{t}$ and a $\boldsymbol{l} \mathrm{x} \boldsymbol{l}$ base. The gridding is fine scale intermediate between the centimeter scale of the filter cake and the micron scale of the individual particles in the deposited layer [4]. The model system is assumed to be flat lying in the $x-y$ plane as shown in Figure 2.1b. 

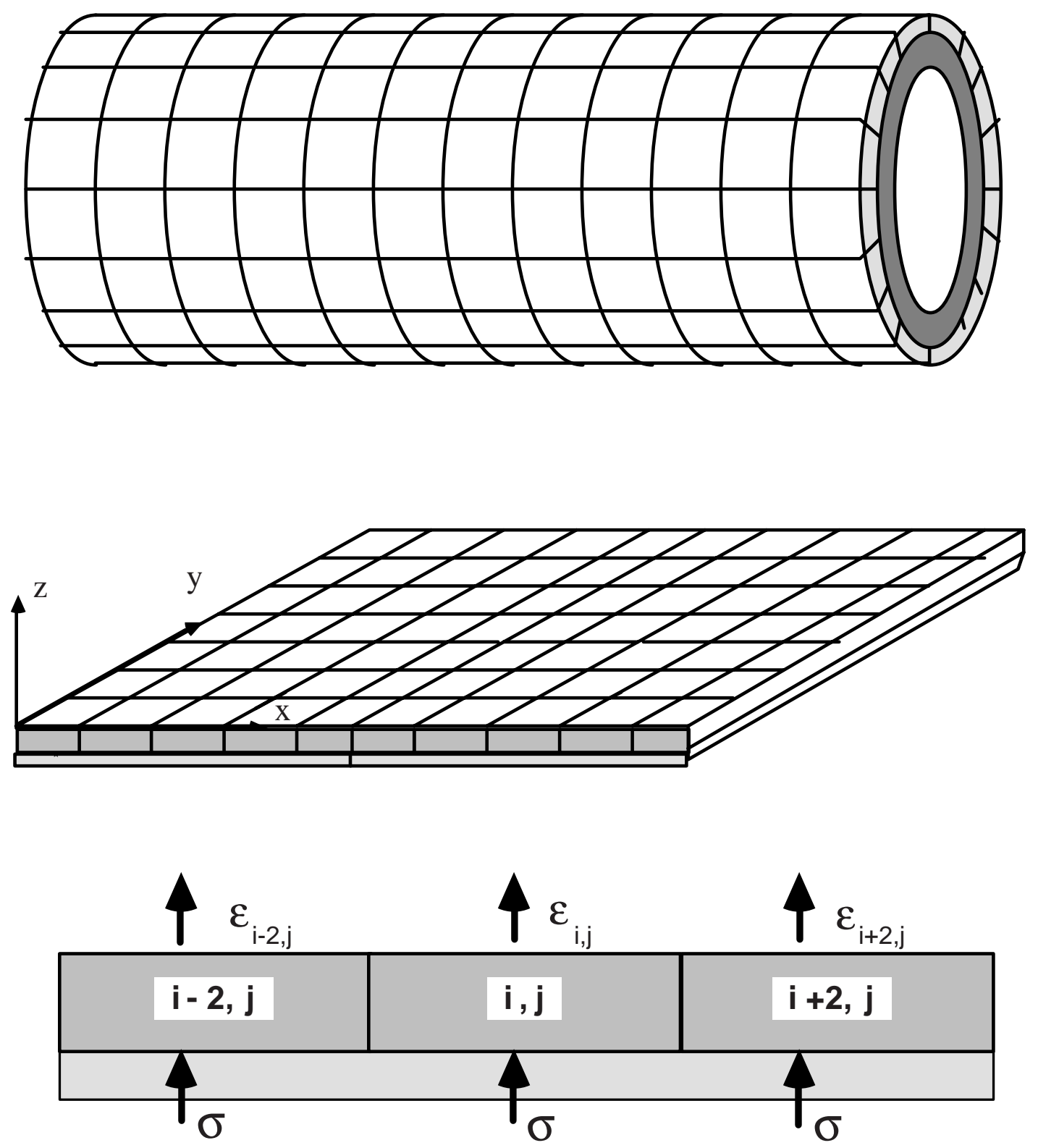

Figure 2.1: Fine-Scale Filter Cake model.

a) The cylindrical filter subdivided into blocks.

b) Planar model of Figure 2.1a, with periodic boundary conditions applied in the $y$ direction to mimic cylindrical symmetry.

c) A small cross-section of Figure 2.1b, showing the applied stress and the resulting displacements. 
To preserve continuity around the cylinder, periodic boundary conditions are applied in the $\mathrm{y}$ direction. The edges in the $\mathrm{x}$ direction are clamped by strong adhesive forces. This prevents these edges from providing artificial defects where failure can initiate. A backpulse cleaning force $\mathrm{F}=P l^{2}$ is applied at the base of each block in a direction perpendicular to the layer as shown in Figure 2.1c. As a result each block is displaced by a small amount $\varepsilon$ in the $\mathrm{z}$ direction. At equilibrium, the applied force $\mathrm{F}$ is balanced by the adhesive force between the block and the filter surface and the cohesive forces between adjacent blocks. The model assumes the adhesive and cohesive forces to be spring like up to a given threshold.

For a block ( $\mathrm{i}, \mathrm{j}$ ) (where $\mathrm{i}$ and $\mathrm{j}$ are even integers which determine location along $\mathrm{x}$ and $\mathrm{y}$ directions respectively), Equation (2.1) provides the basic relation between the force $\mathrm{F}$ applied on the block $(\mathrm{i}, \mathrm{j})$, and the displacements of that block and the surrounding blocks. With the applied force balanced by the adhesive and cohesive spring forces (with spring constants $k^{a}$ and $k^{c}$ respectively), we have,

$\mathrm{F}=k_{i, j}^{a} \varepsilon_{i, j}-\left\{k_{i-1, j}^{c}\left(\varepsilon_{i-2, j}-\varepsilon_{i, j}\right)+k_{i+1, j}^{c}\left(\varepsilon_{i+2, j}-\varepsilon_{i, j}\right)+k_{i, j-1}^{c}\left(\varepsilon_{i, j-2}-\varepsilon_{i, j}\right)+k_{i, j+1}^{c}\left(\varepsilon_{i, j+2}-\varepsilon_{i, j}\right)\right\}$

where each $k^{a}$ is the spring constant of the adhesive spring between the block (i,j) and the substrate, and each $k^{c}$ is the spring constant of the cohesive spring between adjacent blocks. In the simulations undertaken, each spring constant was chosen randomly from a flat distribution of values between 0 and 1 , such that the adhesive springs have an average stiffness of $\frac{1}{2}$ and the cohesive springs have an average stiffness of $\frac{T}{2}$, i.e. $\left\langle k^{a}\right\rangle=\frac{1}{2}$ and $\left\langle k^{c}\right\rangle=\frac{T}{2}$, where $\mathrm{T}$ is the thickness parameter. It gives the ratio of average cohesive force 
to average adhesive force. Since the cohesive strength of a layer, in turn, depends on the thickness $t$ of that layer, therefore the thickness parameter $\mathrm{T}$ also depends on the thickness of the filter cake layer. Equation (2.1) is rearranged to give the displacement $\varepsilon_{i, j}$ of any one block $(\mathrm{i}, \mathrm{j})$,

$\varepsilon_{i, j}=\frac{\left\{k_{i-1, j}^{c} \varepsilon_{i-2, j}+k_{i+1, j}^{c} \varepsilon_{i+2, j}+k_{i, j-1}^{c} \varepsilon_{i, j-2}+k_{i, j+1}^{c} \varepsilon_{i, j+2}\right\}+F}{\left\{k_{i-1, j}^{c}+k_{i+1, j}^{c}+k_{i, j-1}^{c}+k_{i, j+1}^{c}\right\}+k_{i, j}^{a}}$

For a given distribution of stiffness' and the value of F, Equation (2.2) is solved for the displacement of any one block. This is done by initially guessing the values of the displacements $\varepsilon_{i, j}, \varepsilon_{i, j-2}, \varepsilon_{i, j+2}, \varepsilon_{i-2, j}$ and $\varepsilon_{i+2, j}$, and then iterating Equation (2.2) until the displacements stabilize between iterations. If then an adhesive spring is stretched beyond its strength $S^{a}$, i.e.

$k_{i, j}^{a} \varepsilon_{i, j}>S_{i, j}^{a}$

then the adhesive spring breaks. Similarly if a cohesive spring is stretched beyond its strength $S^{c}$, i.e.

$k_{i, j+1}^{c}\left|\varepsilon_{i, j}-\varepsilon_{i, j+2}\right|>S_{i, j+1}^{c}$

then the cohesive spring breaks. As with the spring constants the adhesive strengths $S_{i, j}^{a}$ are chosen such that the average value of the strengths is $\frac{1}{2}$, i.e. $\left\langle S^{a}\right\rangle=\frac{1}{2}$. Similarly the cohesive strengths $S_{i, j+1}^{c}$ are chosen such that their average value is $\frac{T}{2}$, i.e. $\left\langle S^{c}\right\rangle=\frac{T}{2}$. 
Each strength is also strongly correlated with the stiffness, by choosing each strength randomly from a gaussian distribution which is sharply peaked about the value for that spring constant. This ensures that a stiffer spring will have a correspondingly larger strength. The present simulations were undertaken for a time dependent applied force $F(t)$, as will be described in the next chapter.

\subsection{Filter Cake Removal as a Quasi-Static Process}

The time dependence of the filter cake removal (of the order of a few milliseconds) is observed to be much slower than the elastic relaxation of the filter cake (which are of the order of the inverse frequency of elastic waves (sound)). Thus a quasistatic process is justified since the layer has time to reach elastic equilibrium (Equation (2.1)) between successive breaking of bonds [1-4].

\subsection{Computation of the Quasi-Static model}

The computations of the filter cake model proceed in the following steps [1-4],

1) The layer is initially at equilibrium, under no load when the removal force F is applied.

2) Under the influence of the applied force F, the layer reaches a new elastic equilibrium, Equation (2.1). The set of equations for the displacements (Equations 2.2) are iterated, until stabilization is reached.

3) Then each bond weaker than the actual stress is broken, Equations (2.3) and (2.4).

4) Steps (2) and (3) together constitute one time step and are repeated until at a final time step, when no further bonds break.

\subsection{Cascade Effect of Cohesive bonds}

The breaking of some bonds at the end of a time step, puts nearby bonds under greater stress, increasing the likelihood that they will break at the end of the next time step. This cascade effect causes more bonds to break, than would have broken without the interaction 
mediated by the cohesive bonds. Thus the cooperative effects resulting from cohesive bonds produce a cascade that both lowers and sharpens the threshold, i.e. strengthening the cohesive bonds both decreases the removal force at which cleaning occurs and decreases the range of forces required to progress from infinitesimal to complete layer removal [4]. In reality, the cohesive forces may thus prove to be even more significant that the adhesive forces. The thickness parameter $\mathrm{T}$ is a ratio of these two forces, so varying $\mathrm{T}$ varies the relative effect and importance of the adhesive and cohesive forces.

In the following chapter we investigate the effect of cohesive forces and applied pressure on the cleaning efficiency of the filter cake, for each of the 10 cleaning cycles. 


\section{Chapter Three \\ Efficiency of Filter Cake Removal}

\subsection{Introduction}

The fine-scale model is applied to investigate the removal of a filter cake, which is grid into imaginary blocks, with 100 blocks in the y direction and 160 blocks in the $\mathrm{x}$ direction.. For this system the simulations were conducted for three different thickness parameters $\mathrm{T}$ and a range of applied pressures. The thickness parameters studied are,

1) $\mathrm{T}=0.5$ : where the cohesive forces are half as strong as adhesive forces.

2) $\mathrm{T}=1.0$ : where the cohesive forces are as strong as the adhesive forces.

3) $\mathrm{T}=1.5$ : where the cohesive forces are one and a half times as strong as the adhesive forces.

The removal of the filter cake layer was studied through 10 consecutive cleaning cycles where each cycle involved the following steps of layer removal,

1) Initially the layer is at equilibrium. Then a back-pulse pressure $P(t)$ is applied to remove the layer.

2) Under the applied pressure the layer reaches a new elastic equilibrium.

3) All bonds weaker than their respective bond strengths are broken.

4) The layer attains a new elastic equilibrium.

The steps (2) and (3) together constitute one time step. Step (3) and (4) are repeated until no further bonds break. At this point no further removal of filter cake occurs and the cleaning cycle terminates. Before starting the next cycle, the layer is regenerated to the same height in those places where blocks have been removed in the previous cycle. The back pulse pressure $\mathrm{P}(\mathrm{t})$ is applied again and the steps (1) through (4) repeated, till no further bonds break. 
The following sections study the efficiency of layer removal (i.e. the fraction of layer removed) for each of the 10 cleaning cycles, as a function of time and pressure and investigate the effect of cohesion on the cleaning efficiency.

\subsection{Layer removal as a function of Time}

The mass of the deposited layer is defined as the total number of blocks present in the layer. For the model under investigation, the mass of the deposited layer is 16,000 blocks. The fraction of mass removed is defined as the ratio of the number of blocks removed to the total number of blocks present in the system. When an external pressure is applied to removed the layer, then some blocks are removed completely, some blocks adhere to the surface with unbroken adhesive bonds, while some are lifted. In the latter case the adhesive bond breaks, but some cohesive bonds still connect the block to neighboring blocks which remain attached to the substrate.

Figures 3.1(a-c), 3.2(a-c) and 3.3(a-c) show the plots of fraction of mass removed as a function of time step $\mathrm{t}$ for an applied pressure $\mathrm{P}(\mathrm{t})$. Each plot in Figures 3.1, 3.2 and 3.3. shows 5 different realizations, where each realization has the same statistical probability determining spring constants $\left(k^{a}, k^{c}\right)$ and strengths $\left(S^{a}, S^{c}\right)$, but differs in the random number seed. For each realization two sets of computer runs were necessary to determine the data. The first set of runs determined the midpoint pressure $P_{\frac{1}{2}}$, by scanning a range of pressures that would eventually remove half of the deposited layer for that realization at constant pressure.

This midpoint pressure averaged over 5 realizations for each of the three thickness factors is summarized in Table 3.1 on the next page. The data in Table 3.1 suggests that the pressure required to remove half of the deposited layer decreases as the thickness parameter 
increases. This shows that for larger $\mathrm{T}$ (i.e. larger cohesive force), cooperative effects cause nearby bonds to compensate for broken bonds and hence it becomes easier to remove the deposited layer.

\begin{tabular}{|c|c|}
\hline Thickness Parameter T & Midpoint Pressure $P_{\frac{1}{2}}$ \\
\hline 0.5 & 0.285172 \\
1.0 & 0.269076 \\
1.5 & 0.257552 \\
\hline
\end{tabular}

Table 3.1: Thickness parameters and their corresponding midpoint pressures.

On determining the midpoint pressures for each realization for a given thickness parameter $\mathrm{T}$, the second set of runs is performed to determine the mass of layer removed as a function of time step $\mathrm{t}$ for each realization. The time dependent pressure $\mathrm{P}(\mathrm{t})$ applied to remove the layer is given as,

$\mathrm{P}(\mathrm{t})=\left(P_{\frac{1}{2}}+\mathrm{dP}\right)(1-\mathrm{f})$

where $\mathrm{dP}$ is a small increment or decrement to the midpoint pressure, $\mathrm{f}$ is the fraction of mass that has been removed by time step t, and $P_{\max }=P_{\frac{1}{2}}+\mathrm{dP}$ is the value of the pressure pulse at the start of each cycle $(\mathrm{t}=0, \mathrm{f}=0)$.

The resulting plots of fraction of mass removed as a function of time step for a given applied pressure pulse $\mathrm{P}(\mathrm{t})$, are as shown in Figures $3.1(\mathrm{a}-\mathrm{c}), 3.2(\mathrm{a}-\mathrm{c})$ and $3.3(\mathrm{a}-\mathrm{c})$ in the pages that follow. 


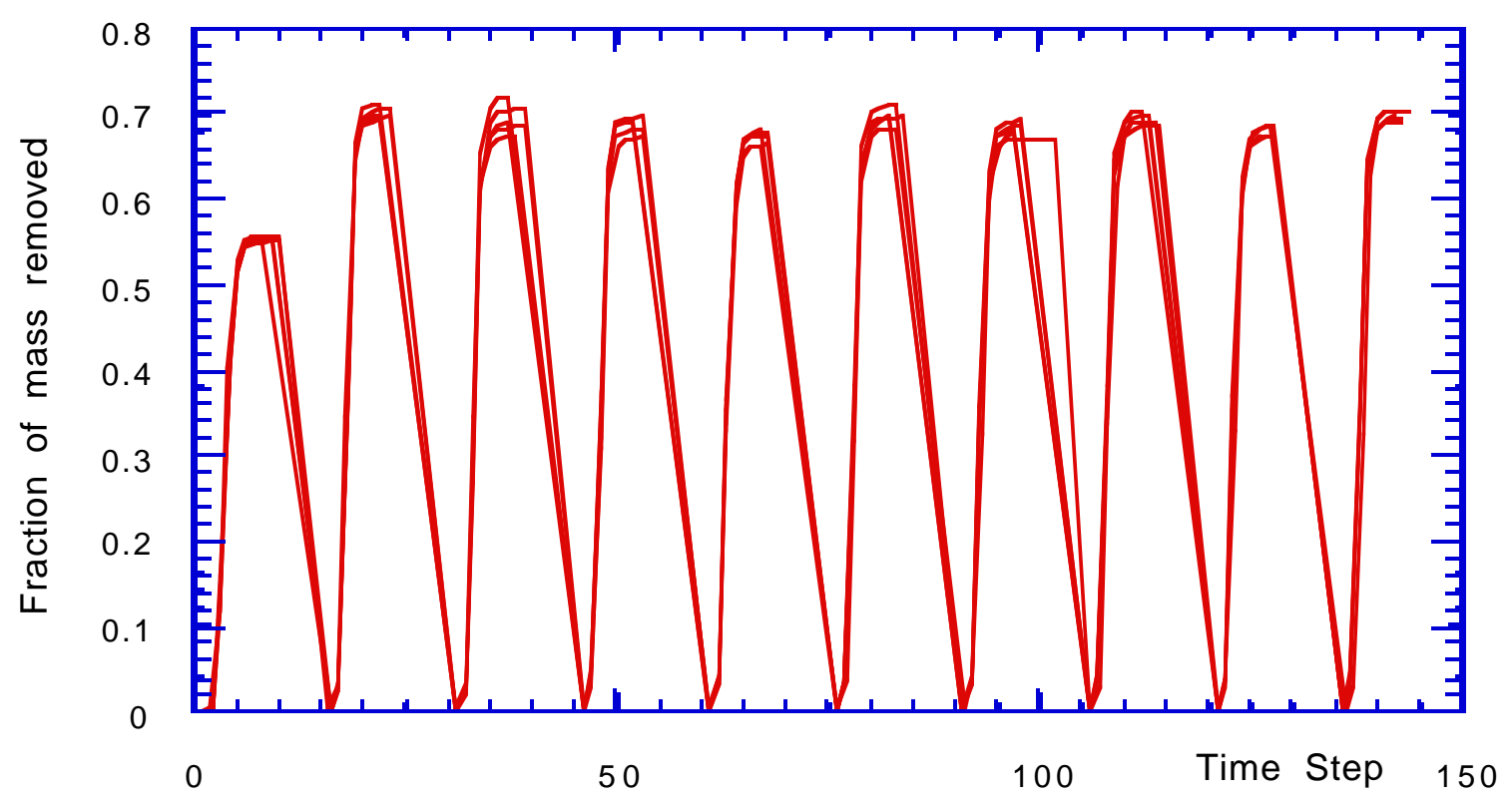

Figure 3.1a: Plot of Fraction of mass removed Vs. Time step, for thickness parameter $\mathrm{T}=0.5$ and pressure $\mathrm{P}(\mathrm{t})=\left(P_{\frac{1}{2}}+0.04\right)(1-\mathrm{f})$ for all 10 cleaning cycles. $($ Here $\mathrm{dP}=0.04)$.

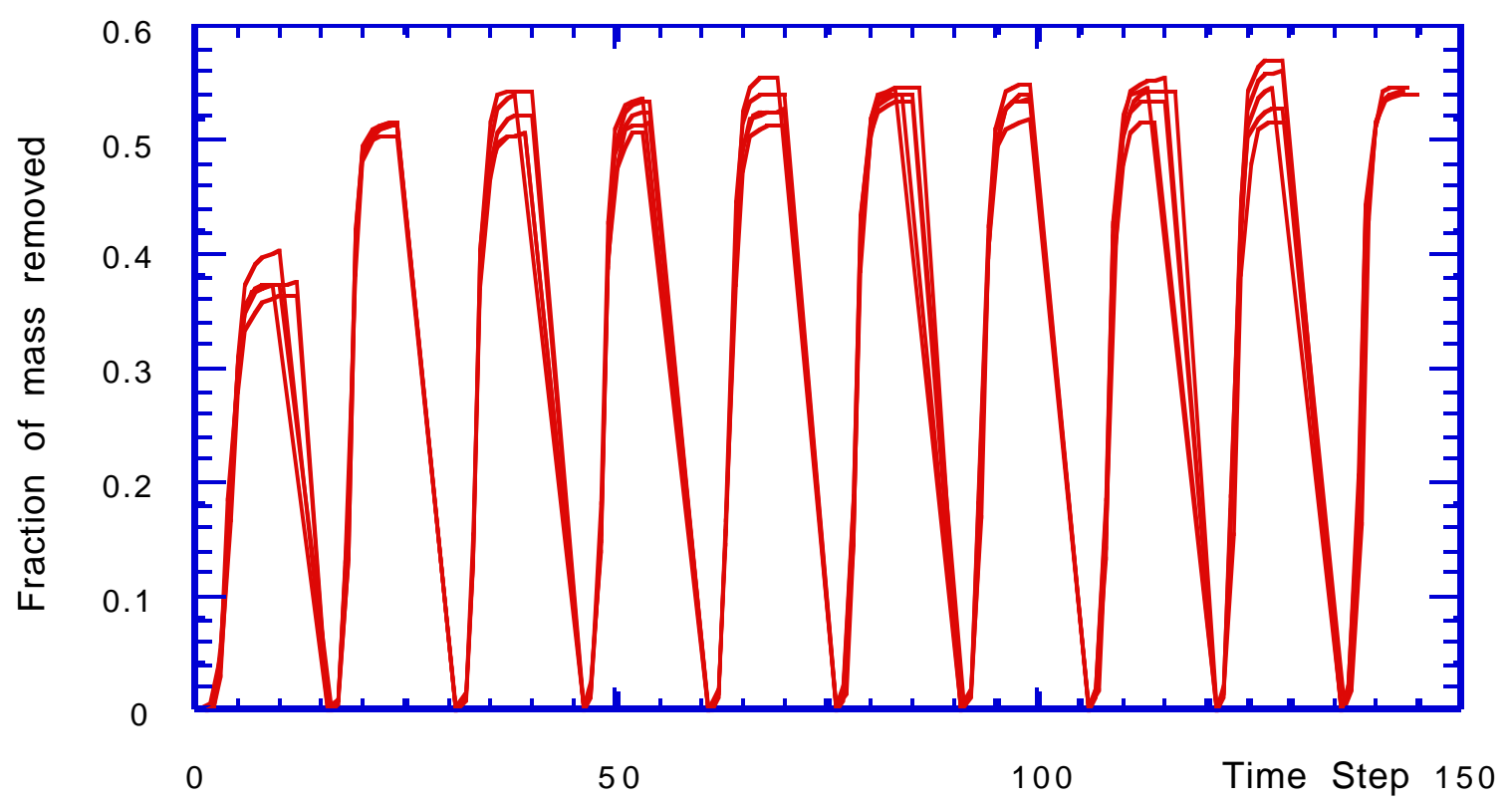

Figure 3.1b: Plot of Fraction of mass removed Vs. Time step, for thickness parameter $\mathrm{T}=0.5$ and pressure $\mathrm{P}(\mathrm{t})=\left(P_{\frac{1}{2}}+0.01\right)(1-\mathrm{f})$ for all 10 cleaning cycles. (Here $\left.\mathrm{dP}=0.01\right)$. 


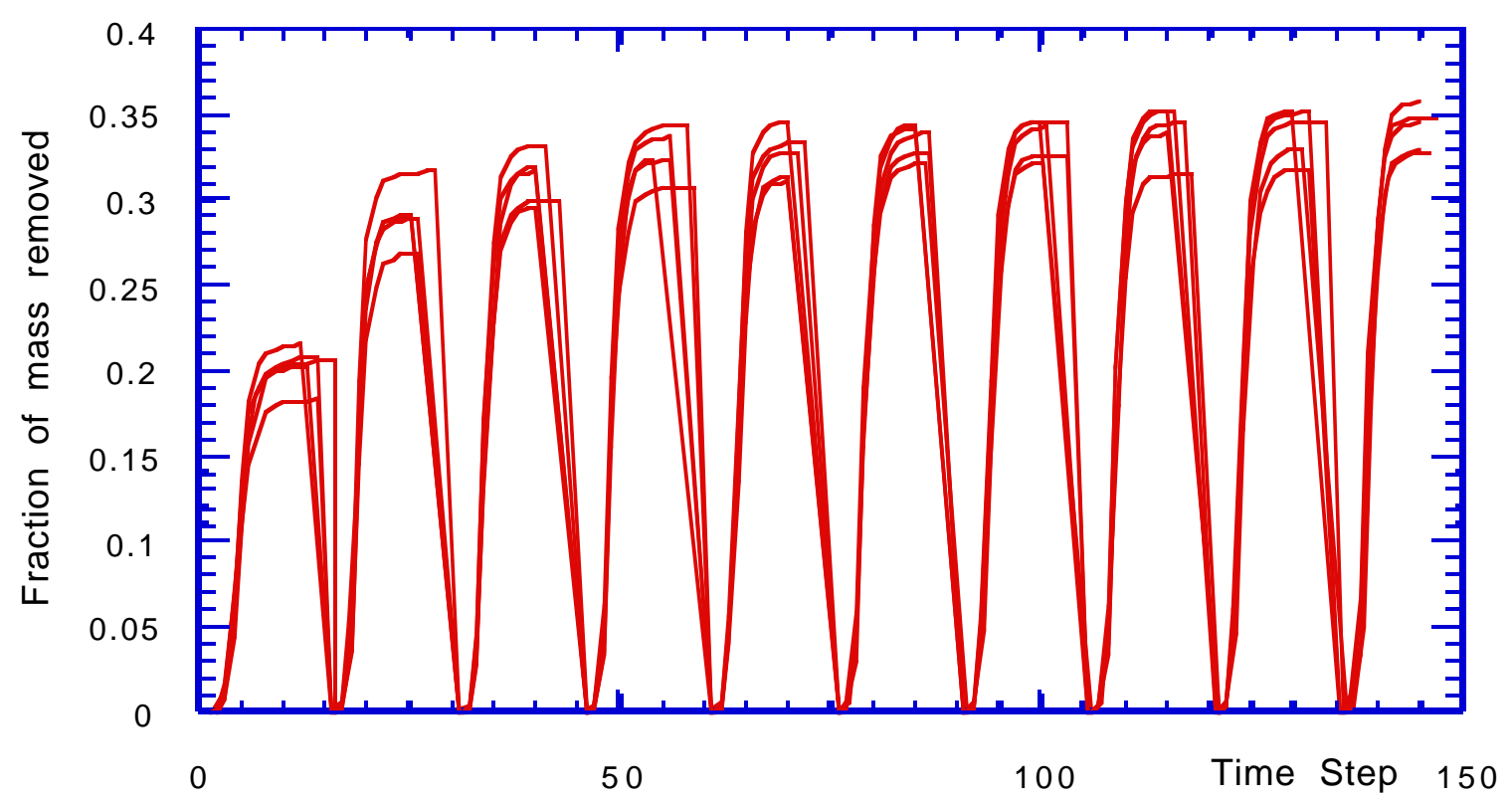

Figure 3.1c: Plot of Fraction of mass removed Vs. Time step, for thickness parameter $\mathrm{T}=0.5$ and pressure $\mathrm{P}(\mathrm{t})=\left(P_{\frac{1}{2}}-0.02\right)(1-\mathrm{f})$ for all 10 cleaning cycles. (Here $\left.\mathrm{dP}=-0.02\right)$.

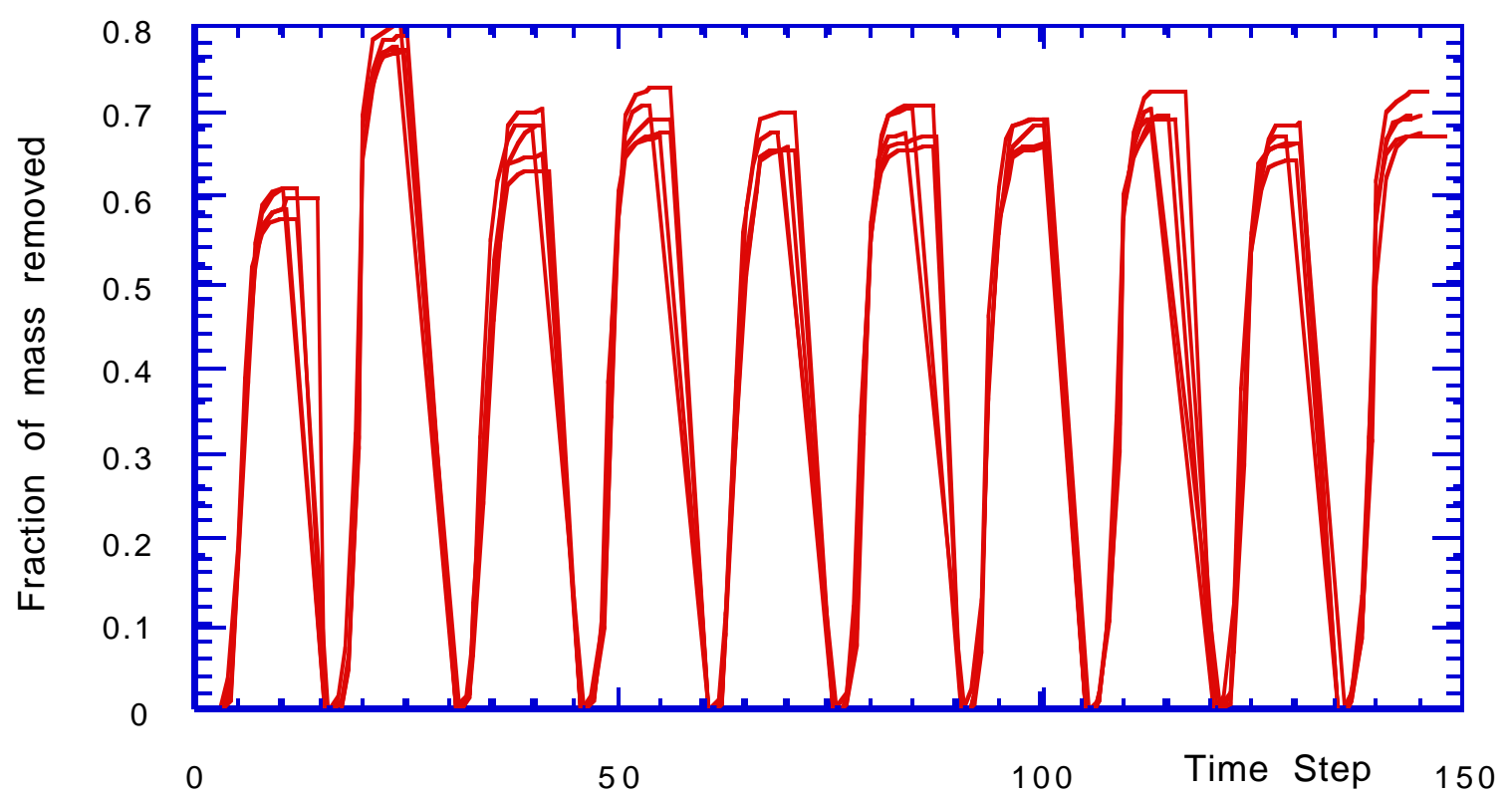

Figure 3.2a: Plot of Fraction of mass removed Vs. Time step, for thickness parameter $\mathrm{T}=1.0$ and pressure $\mathrm{P}(\mathrm{t})=\left(P_{\frac{1}{2}}+0.04\right)(1-\mathrm{f})$ for all 10 cleaning cycles. $($ Here $\mathrm{dP}=0.04)$. 


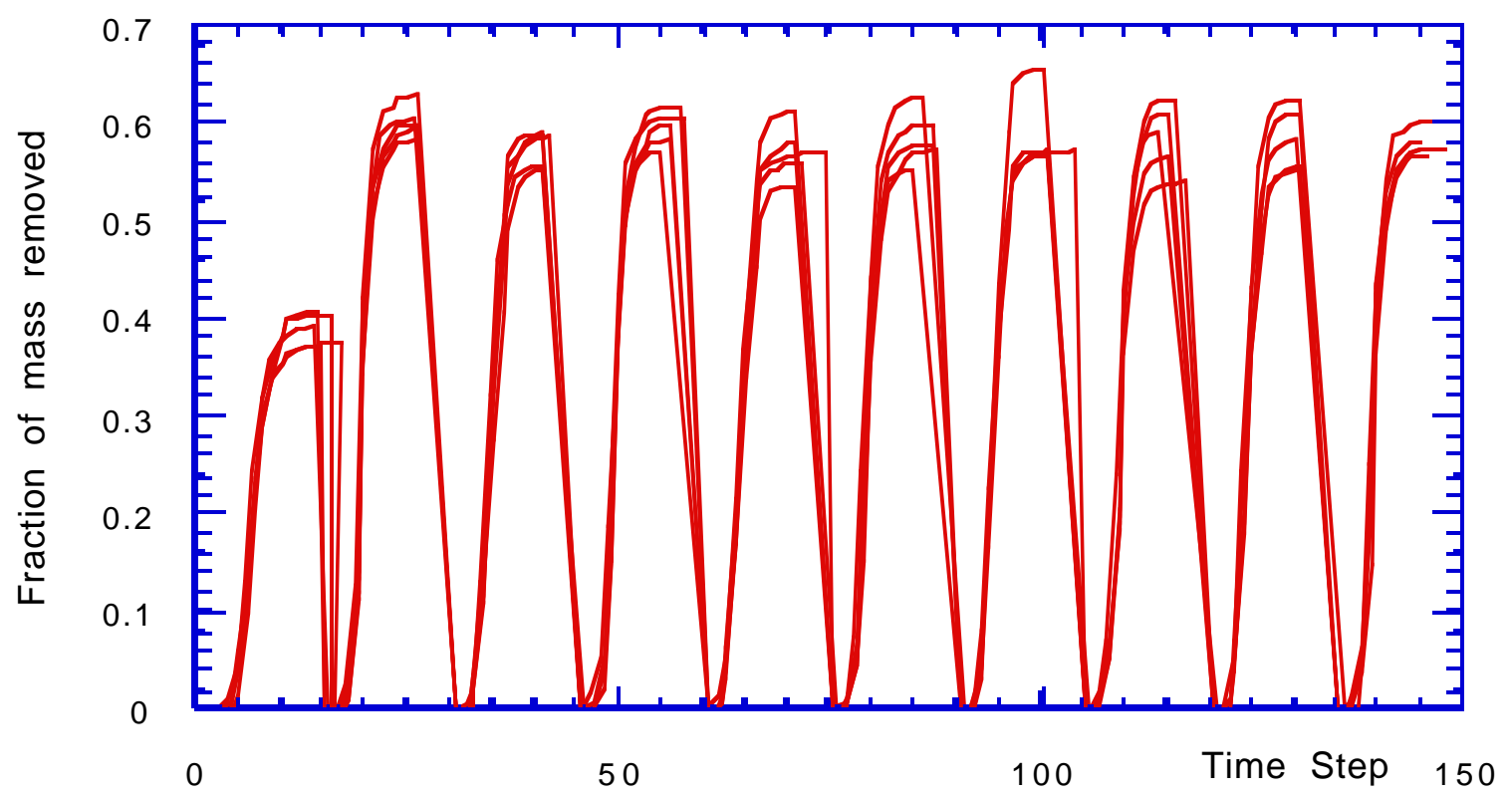

Figure 3.2b: Plot of Fraction of mass removed Vs. Time step, for thickness parameter $\mathrm{T}=1.0$ and pressure $\mathrm{P}(\mathrm{t})=\left(P_{\frac{1}{2}}+0.02\right)(1-\mathrm{f})$ for all 10 cleaning cycles. (Here $\left.\mathrm{dP}=0.02\right)$.

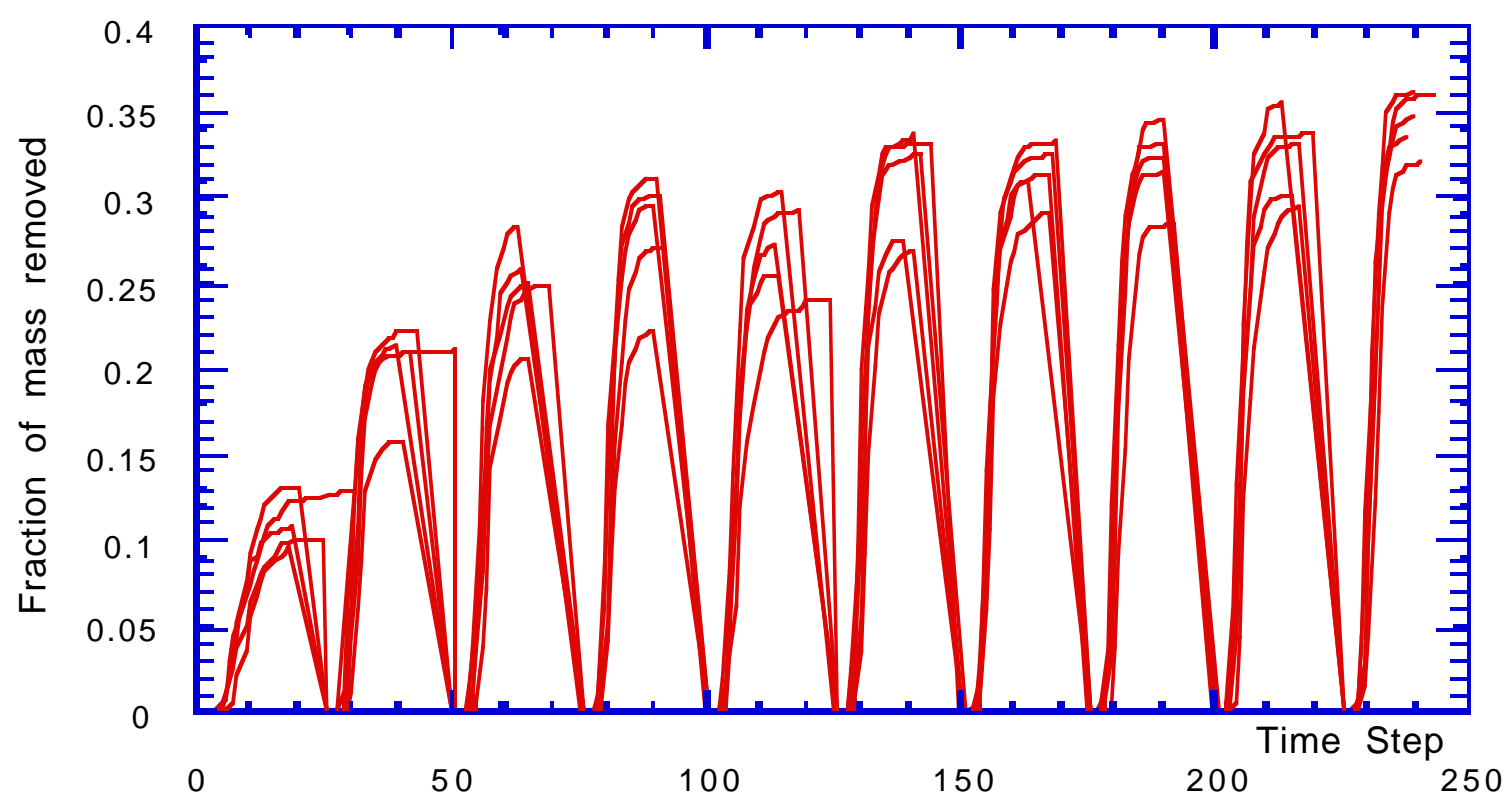

Figure 3.2c: Plot of Fraction of mass removed Vs. Time step, for thickness parameter $\mathrm{T}=1.0$ and pressure $\mathrm{P}(\mathrm{t})=\left(P_{\frac{1}{2}}-0.01\right)(1-\mathrm{f})$ for all 10 cleaning cycles. $($ Here $\mathrm{dP}=-0.01)$. 


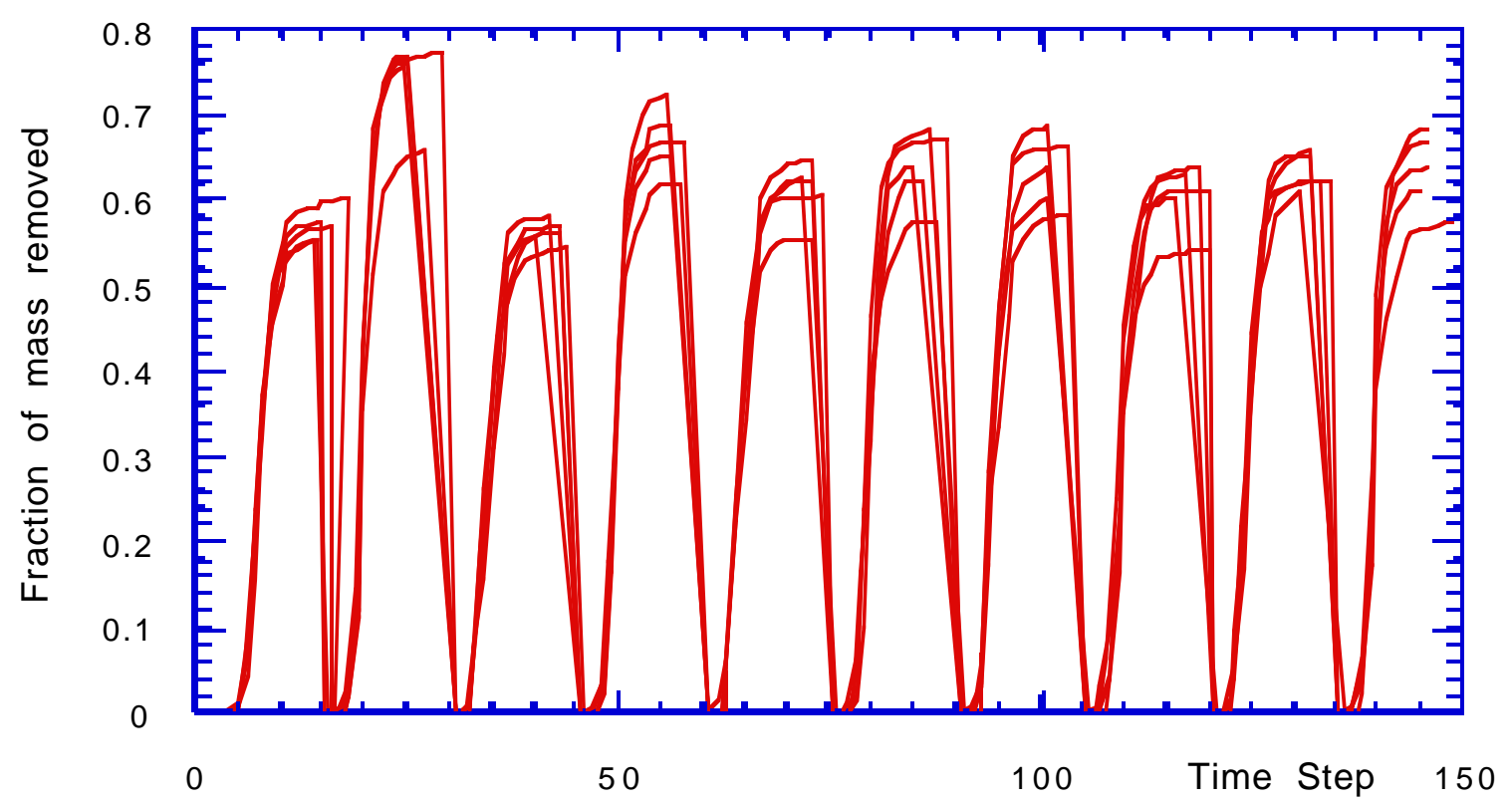

Figure 3.3a: Plot of Fraction of mass removed Vs. Time step, for thickness parameter $\mathrm{T}=1.5$ and pressure $\mathrm{P}(\mathrm{t})=\left(P_{\frac{1}{2}}+0.04\right)(1-\mathrm{f})$ for all 10 cleaning cycles. (Here $\left.\mathrm{dP}=0.04\right)$.

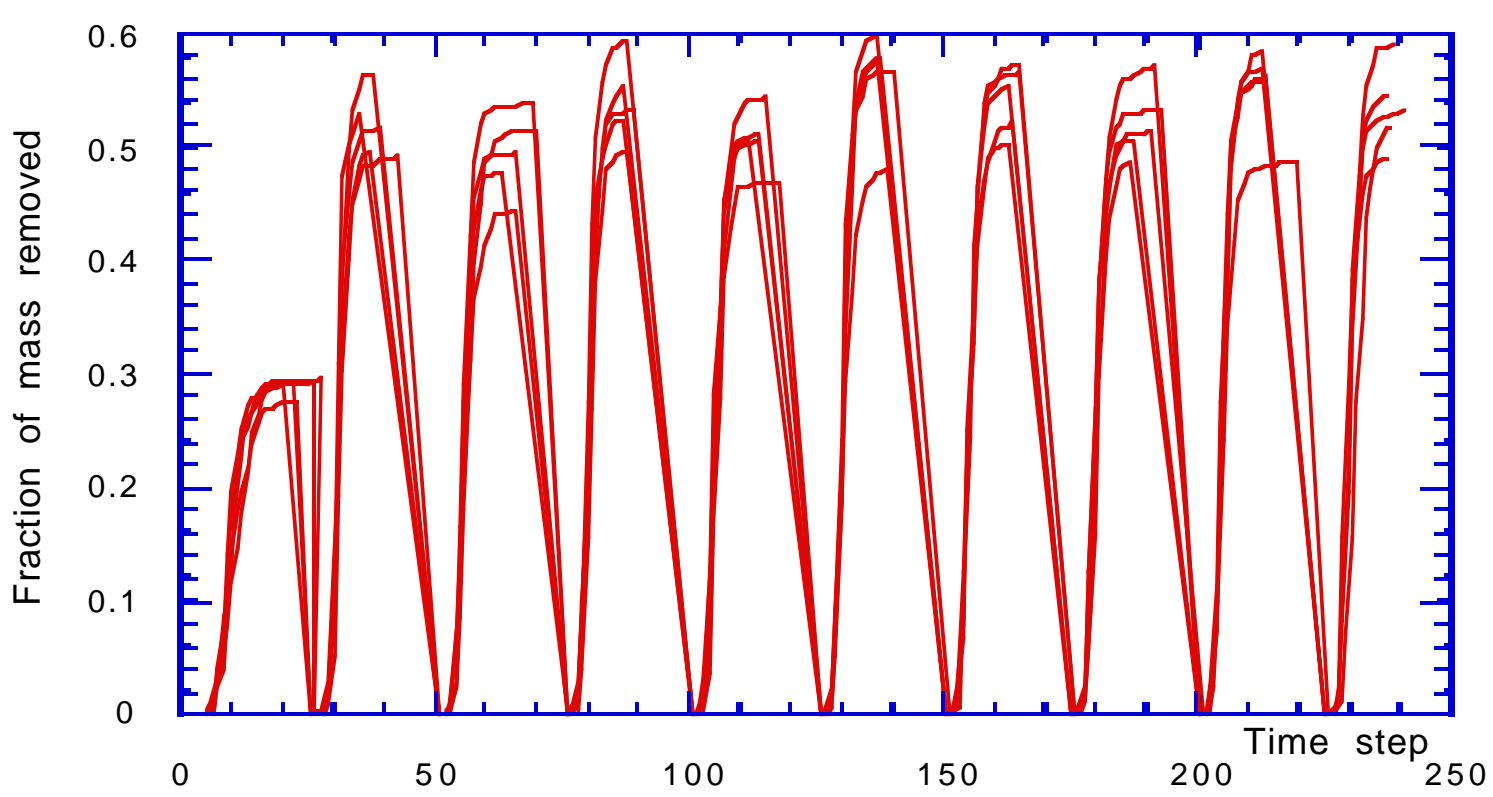

Figure 3.3b: Plot of Fraction of mass removed Vs. Time step, for thickness parameter $\mathrm{T}=1.5$ and pressure $\mathrm{P}(\mathrm{t})=\left(P_{\frac{1}{2}}+0.02\right)(1-\mathrm{f})$ for all 10 cleaning cycles. $($ Here $\mathrm{dP}=0.02)$. 


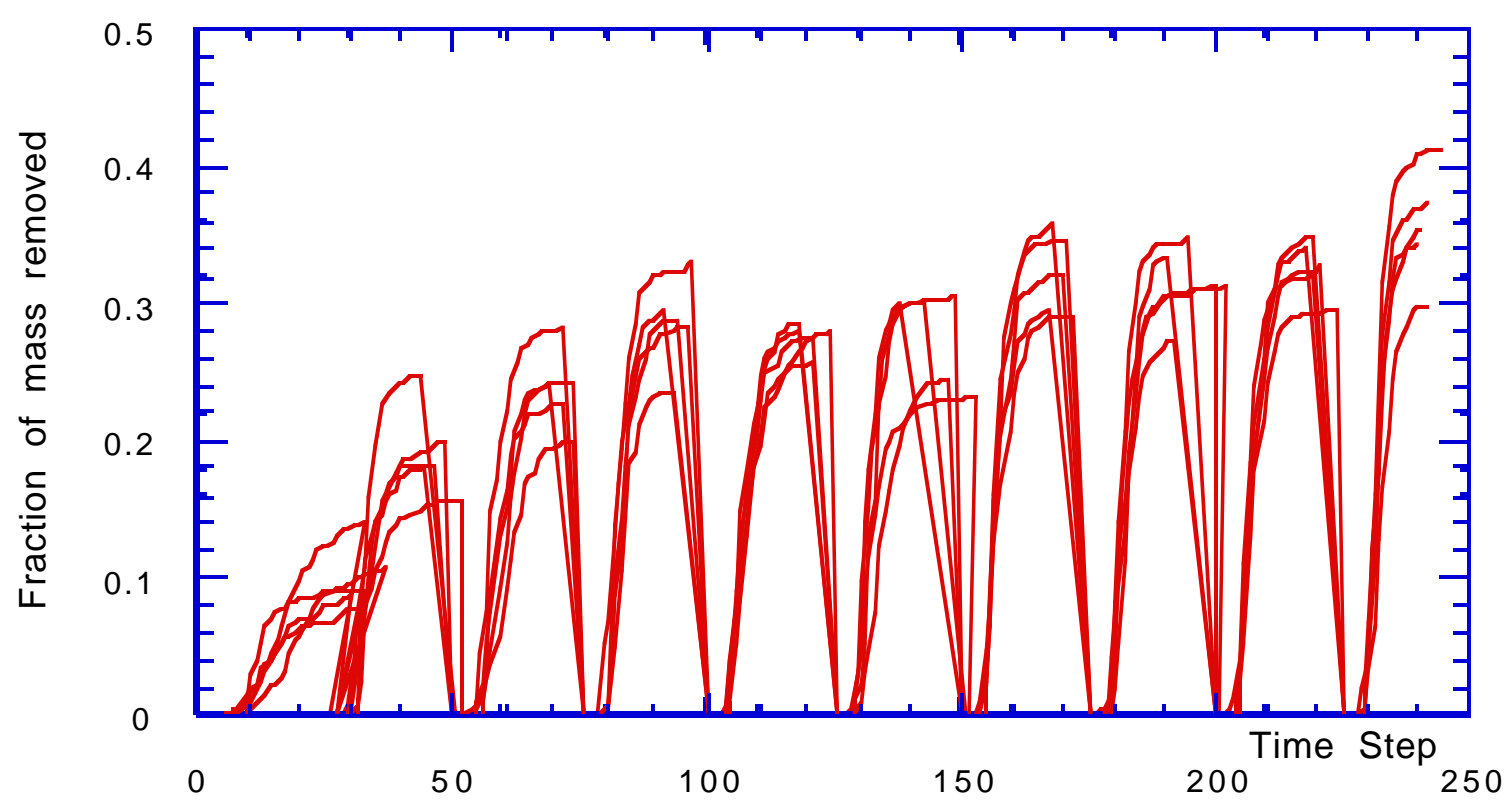

Figure 3.3c: Plot of Fraction of mass removed Vs. Time step, for thickness parameter $\mathrm{T}=1.5$ and pressure $\mathrm{P}(\mathrm{t})=\left(P_{\frac{1}{2}}+0.0\right)(1-\mathrm{f})$ for all 10 cleaning cycles. $($ Here $\mathrm{dP}=0.0)$.

\subsection{Discussion of Plots}

The time axis of the above plots of Figures 3.1, 3.2 and 3.3 comprise 10 cycles, where the cycles are as described in Section 1.1. At the start of each cleaning cycle $(t=0, f=0)$, the applied pressure $\mathrm{P}(\mathrm{t})$ given by Equation (3.1), has a maximum value of $P_{\max }=P_{\frac{1}{2}}+\mathrm{dP}$. As time progresses, more and more mass is removed and the pressure pulse decreases in magnitude as can be seen by Equation (3.1). During early time steps of each cycle no filter cake may be removed even as the weakest adhesive bonds are being broken. This is because a block may still be held by cohesive forces even though its adhesive bond has broken. For each cleaning cycle this short per-cleaning period during which no mass is removed has been observed in the simulations $[1,4]$. The applied pressure now causes the weakest cohesive bonds (which are already under greater stress due to broken adhesive bonds) to break, and mass begins to be removed at a fairly uniform pace until a limiting 
value, which depends on the maximum applied pressure $P_{\max }$. Before starting the next cycle the layer is regenerated (to the same height) in those areas where blocks have been removed in the previous cycle. The resulting layer now has places where blocks have been lifted but not removed. This damage makes these areas weaker and thus they are more likely to be removed in the next cycle.

The plots of Figures 3.1-3.3 show that less mass is removed in the first cycle, than in later cycles. This occurs because at the start of the second cycle the blocks which are lifted but not removed, are more weakly bound, (since they have broken adhesive bonds, and some unbroken cohesive bonds), and are hence more likely to be removed in the second cycle. Thus in the second cycle additional mass is removed. As the number of cycles increases the fraction of mass removed is seen to stabilize, because not all of the blocks that are lifted in a given cycle $n$ are removed in the next cycle $n+1$. The limiting value of fraction of mass removed, is averaged over the last 5 cycles, over all 5 realizations and the results are listed in tables 3.2(a-c).

\begin{tabular}{|c|c|}
\hline Differential Pressure dP & $\begin{array}{c}\text { Limiting value of Fraction of Mass removed } \\
\text { (Average over last 5 cycles) }\end{array}$ \\
\hline 0.04 & 0.6896 \\
0.03 & 0.6462 \\
0.02 & 0.5932 \\
0.01 & 0.5398 \\
-0.01 & 0.4084 \\
-0.02 & 0.3382 \\
-0.03 & 0.2652 \\
-0.04 & 0.1968 \\
\hline
\end{tabular}

Table 3.2a: Differential pressures and the corresponding limiting values of fraction of mass removed, for thickness parameter $\mathrm{T}=0.5$. 


\begin{tabular}{|c|c|}
\hline $\begin{array}{c}\text { Differential Pressure dP } \\
\text { Limiting value of Fraction of Mass removed } \\
\text { (Average over last 5 cycles) }\end{array}$ \\
\hline 0.04 & 0.6833 \\
0.03 & 0.6347 \\
0.02 & 0.5844 \\
0.01 & 0.5213 \\
-0.01 & 0.3224 \\
-0.015 & 0.2486 \\
-0.02 & 0.1912 \\
-0.025 & 0.1382 \\
\hline
\end{tabular}

Table 3.2b: Differential pressures and the corresponding limiting values of fraction of mass removed, for thickness parameter $\mathrm{T}=1.0$.

\begin{tabular}{|c|c|}
\hline Differential Pressure dP & $\begin{array}{c}\text { Limiting Value of Fraction of Mass removed } \\
\text { (Average over last 5 cycles) }\end{array}$ \\
\hline 0.04 & 0.6287 \\
0.03 & 0.5982 \\
0.02 & 0.5423 \\
0.0 & 0.3197 \\
-0.005 & 0.2328 \\
-0.01 & 0.1480 \\
\hline
\end{tabular}

Table 3.2c: Differential pressures and the corresponding limiting values of fraction of mass removed, for thickness parameter $\mathrm{T}=1.5$.

\subsection{Layer Removal as a function of Pressure}

The data in Tables 3.2 (a-c) is used to plot Figure 3.4 as shown on the next page. The figure plots the limiting value of fraction of mass removed (averaged over last 5 cycles) against the maximum applied pressure $P_{\max }$, for the 3 thickness parameters under 
consideration. The plot shows that for a particular thickness parameter the fraction of mass removed increases with the maximum applied pressure that is applied. Thus for greater applied pressures more layer mass is removed as expected.

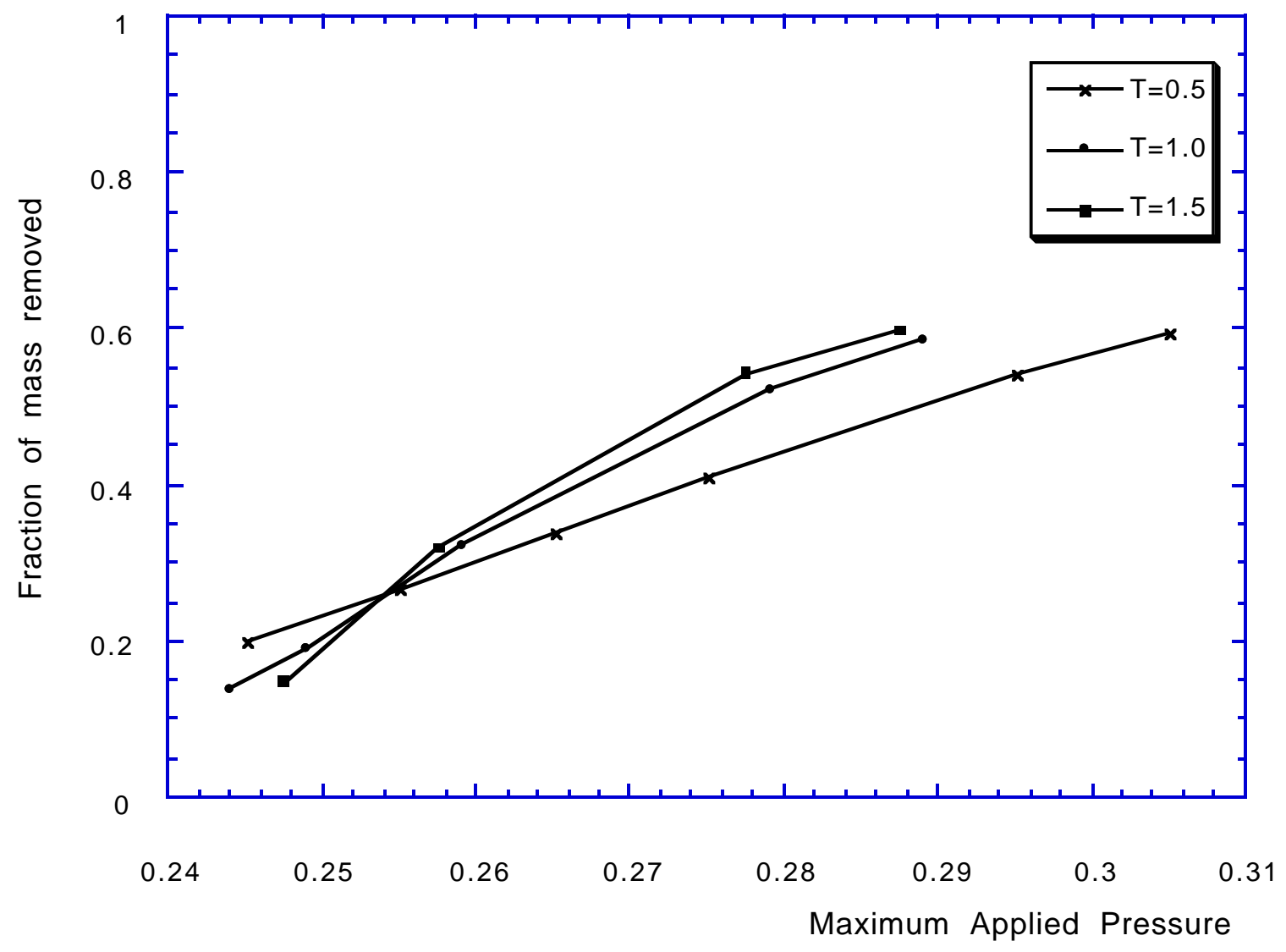

Figure 3.4: Plot of Fraction of mass removed Vs. Maximum applied Pressure $P_{\max }=P_{\frac{1}{2}}+$ $\mathrm{dP}$, for the 3 thickness parameters under consideration.

A comparison of the trends among the three thickness parameter plots shows that for higher values of the applied pressure (greater than 0.254) the fraction of mass removed (at a given value of applied pressure), is greater for higher values of T. This implies that stronger 
cohesive forces introduce cooperative effects resulting in more mass being removed i.e. for a thicker cake more layer mass is removed at higher applied pressures. For lower values of applied pressure (less than 0.254 ) the trend reverses. The fraction of mass removed (at a given value of applied pressure) is reduced for higher values of $\mathrm{T}$. This behavior suggests that for smaller applied pressures it is more difficult to remove blocks which are connected to neighboring blocks by strong cohesive forces.

\subsection{Conclusion}

The simulations show that the cooperative effects introduced by the cohesive forces play an important role in filter cake removal in that they significantly lower the pressure that has to be applied to remove the layer, and also cause more mass to be removed for thicker (more cohesive) cakes. For each cleaning cycle the partial removal of the filter cake at each time step, increases the permeability of the filter, requiring a reduced pressure drop to maintain constant flow. This is ensured by using a time dependent pressure pulse $P(t)$ in the simulations. An understanding of these effects allows for filter cake removal to occur at lower applied pressures, making the operation more economical.

In the next chapter, we study the effect of adhesion as the cleaning proceeds from one cycle to the next. 


\section{Chapter Four \\ Cleaning Cycle Analysis I}

\subsection{Introduction}

The fine-scale model is used to simulate cleaning of a cylindrical filter through 10 consecutive cycles. Each cleaning cycle results in some (but not all) of the filter cake being removed. Before starting the next cleaning cycle, the layer is regenerated to the same height in those areas where the filter cake has been removed, but left unchanged in those areas where the filter cake has not been removed, in the previous cycle. The latter regions contain some broken adhesive bonds which correspond to blocks that have been lifted but not removed in the previous cycle. Thus at the start of a new cycle, the damaged adhesive bonds have not been repaired, making these blocks weaker and more likely to be removed in the next cycle. This chapter analyzes the trends in filter cake removal as the cleaning proceeds from one cycle to the next and examines the role of the broken adhesive bonds in the cleaning process.

\subsection{Blocks which are removed in two consecutive cycles}

A computer program is written to count the total number of blocks removed in each cycle and to calculate the fraction of those blocks removed in cycle n, which are also removed in cycle $n+1$. This fraction is plotted against the cycle number $n$ in Figures 4.1-4.9, for the three thickness parameters under consideration and for a variety of applied pressures. Figures 4.1a-4.9a plot the total number of blocks removed in a given cycle $\mathrm{n}$, while Figures $4.1 \mathrm{~b}-4.9 \mathrm{~b}$ plot the fraction of those blocks removed in cycle $\mathrm{n}$, which are also removed in cycle $n+1$. To facilitate observation of trends, the results of Figures $4.1 b-4.9 b$ are summarized in Figure 4.10, which shows the fraction of blocks removed in two successive cycles (averaged over the last few cycles). 


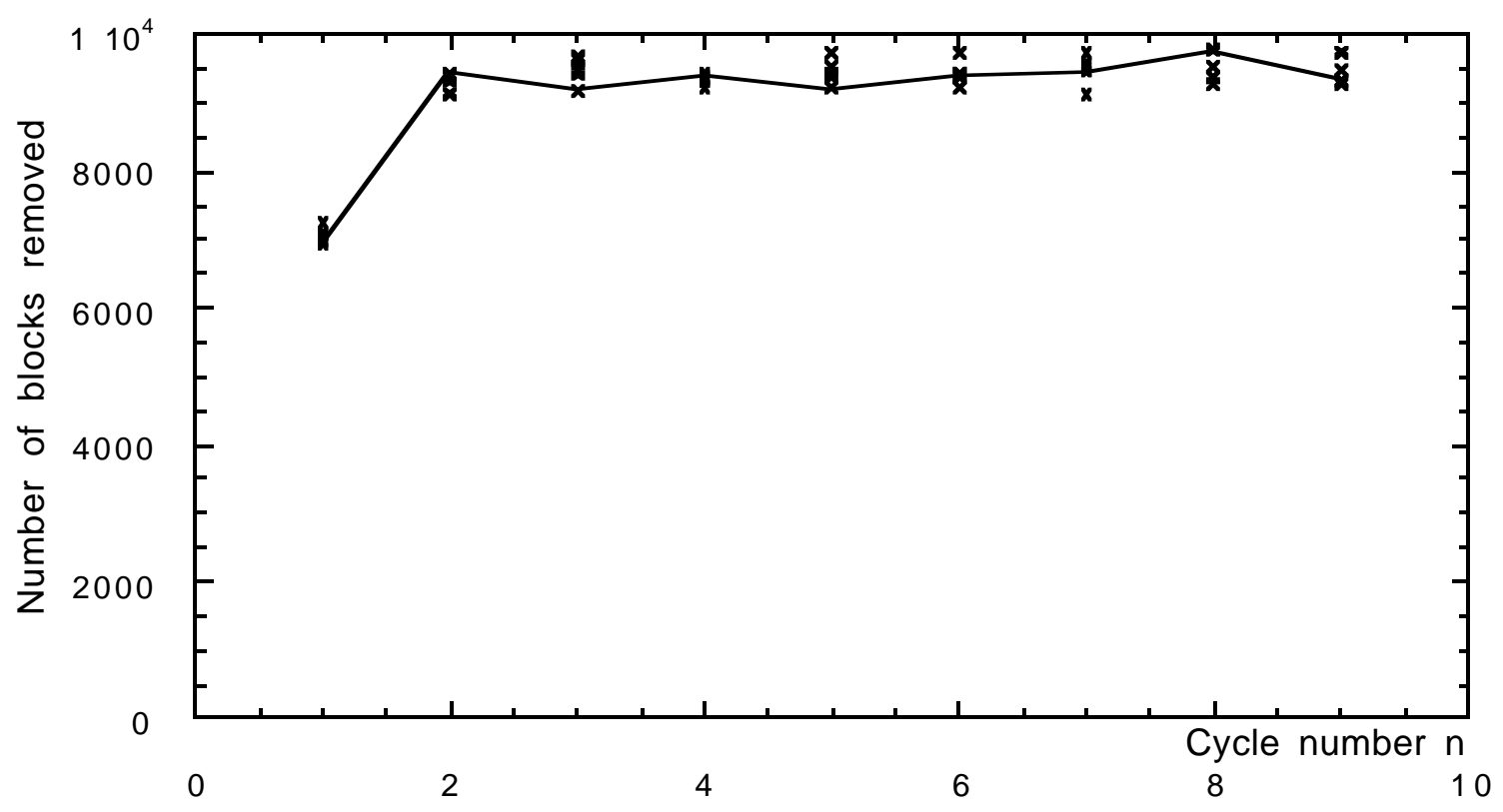

Figure 4.1a: Plot of the total number of blocks removed in cycle $n$ Vs. the cycle number $n$, for thickness parameter T=0.5 and maximum pressure $P_{\max }=P_{\frac{1}{2}}+0.02$ for each cycle.

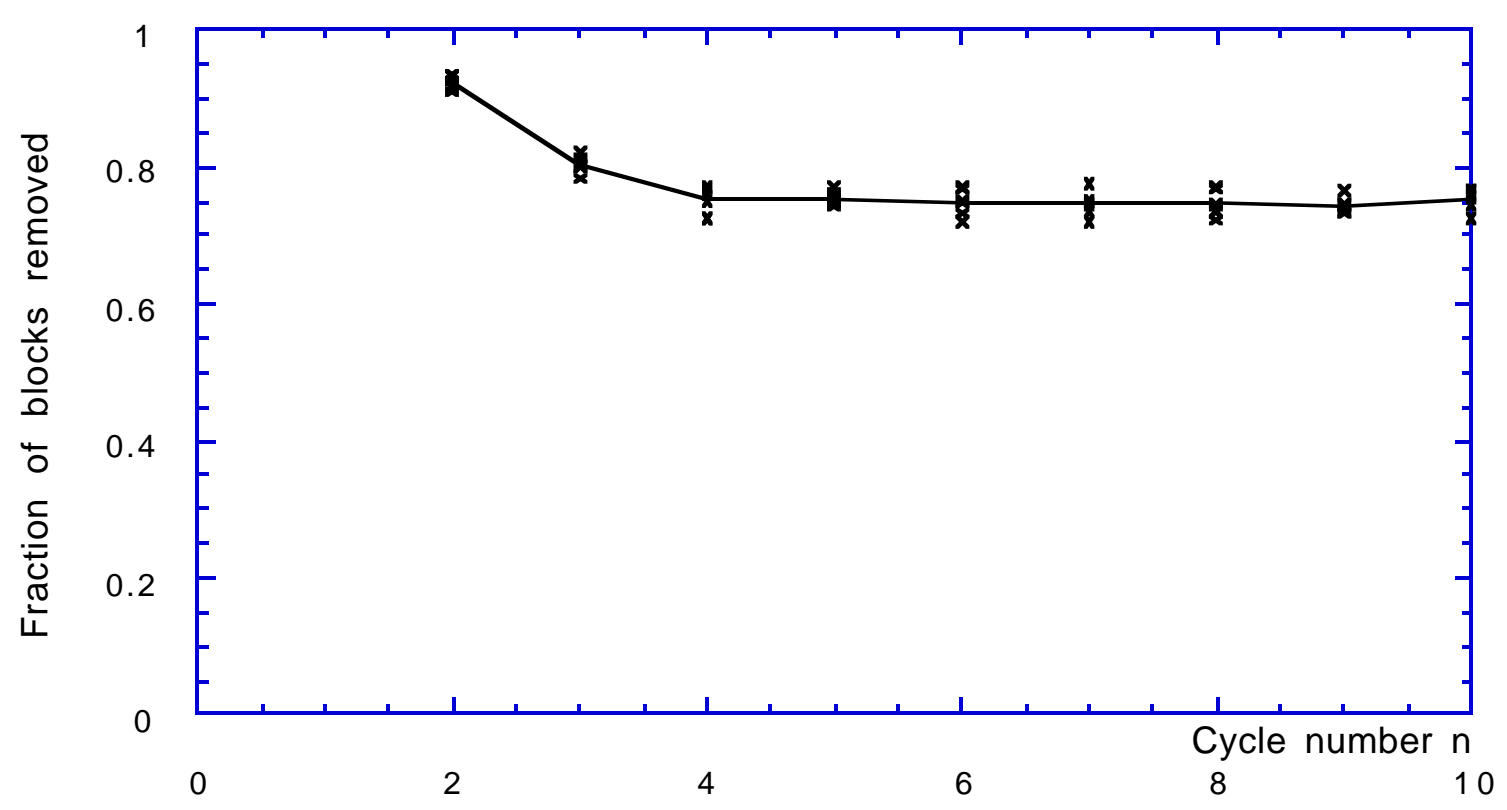

Figure 4.1b: Plot of Fraction of those blocks removed in cycle n, which are also removed in cycle $\mathrm{n}+1$ Vs. cycle number $\mathrm{n}$, for $\mathrm{T}=0.5$ and $P_{\max }=P_{\frac{1}{2}}+0.02$ for each cycle. 


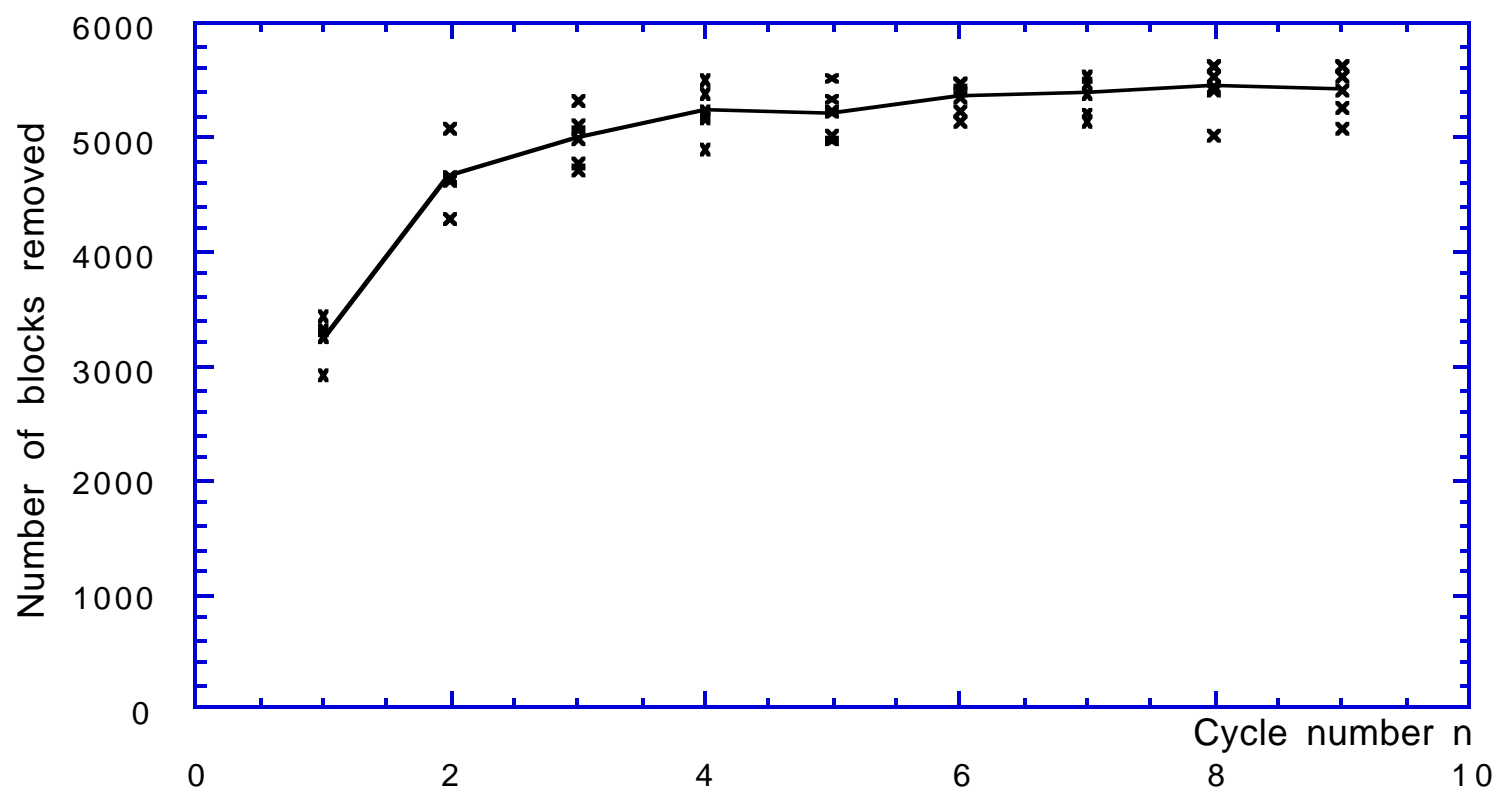

Figure 4.2a: Plot of the total number of blocks removed in cycle $n$ Vs. the cycle number $n$, for thickness parameter $\mathrm{T}=0.5$ and maximum of pressure $P_{\max }=P_{\frac{1}{2}}-0.02$ for each cycle.

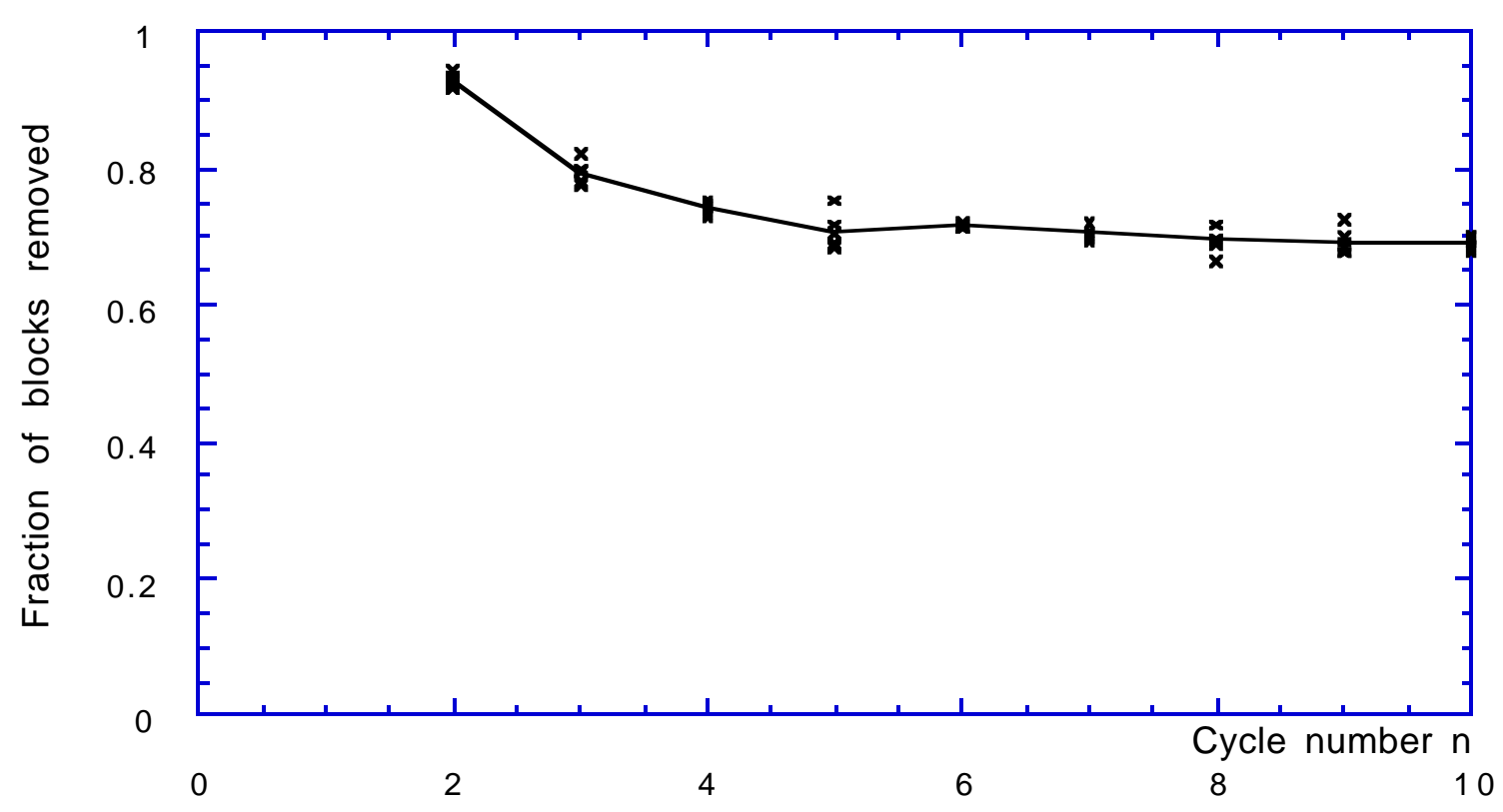

Figure 4.2b: Plot of Fraction of those blocks removed in cycle $n$, which are also removed in cycle $\mathrm{n}+1$ Vs. cycle number $\mathrm{n}$, for $\mathrm{T}=0.5$ and $P_{\max }=P_{\frac{1}{2}}-0.02$ for each cycle. 


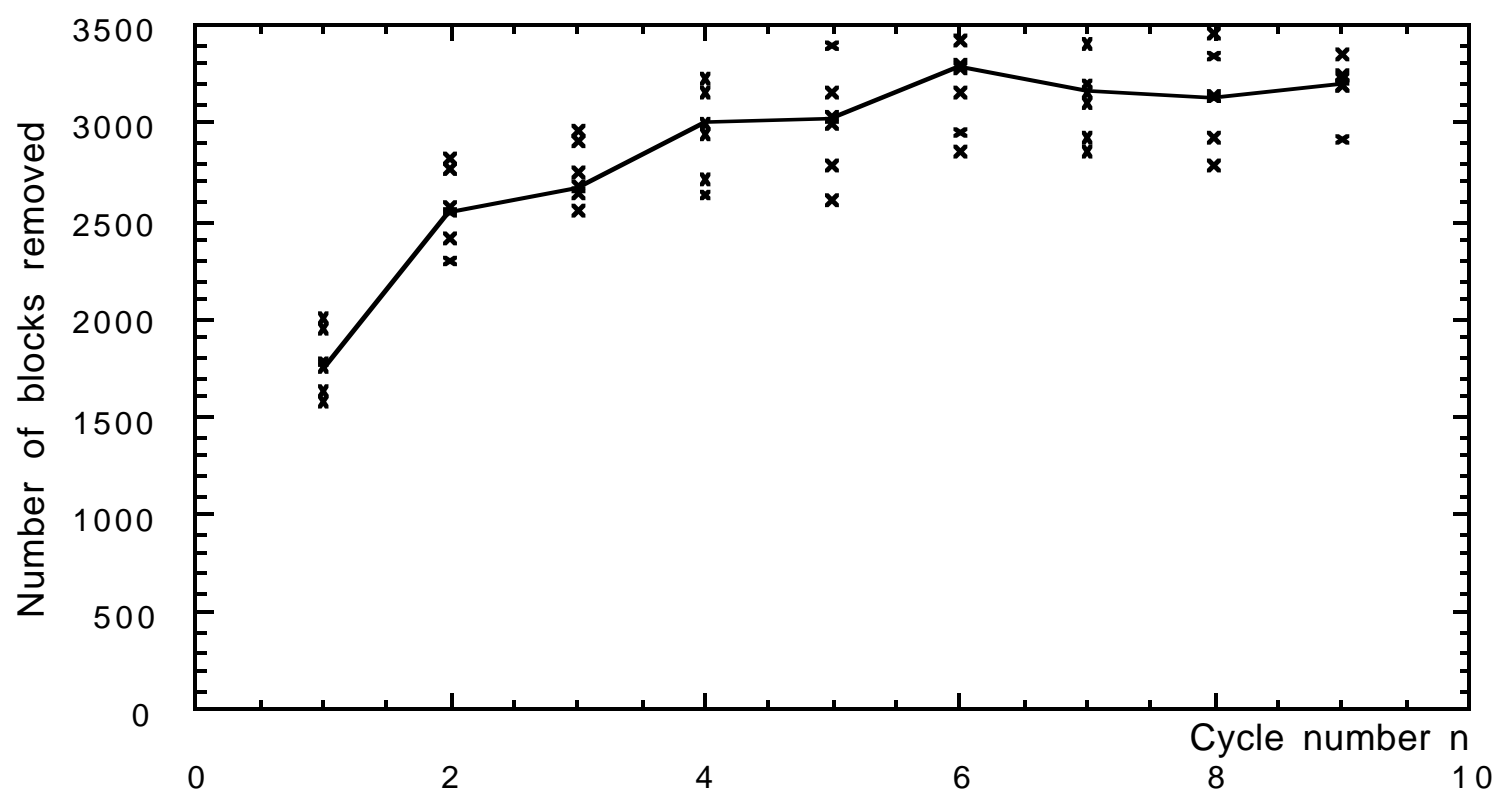

Figure 4.3a: Plot of the total number of blocks removed in cycle $n$ Vs. the cycle number n, for thickness parameter $\mathrm{T}=0.5$ and maximum pressure $P_{\max }=P_{\frac{1}{2}}-0.04$ for each cycle.

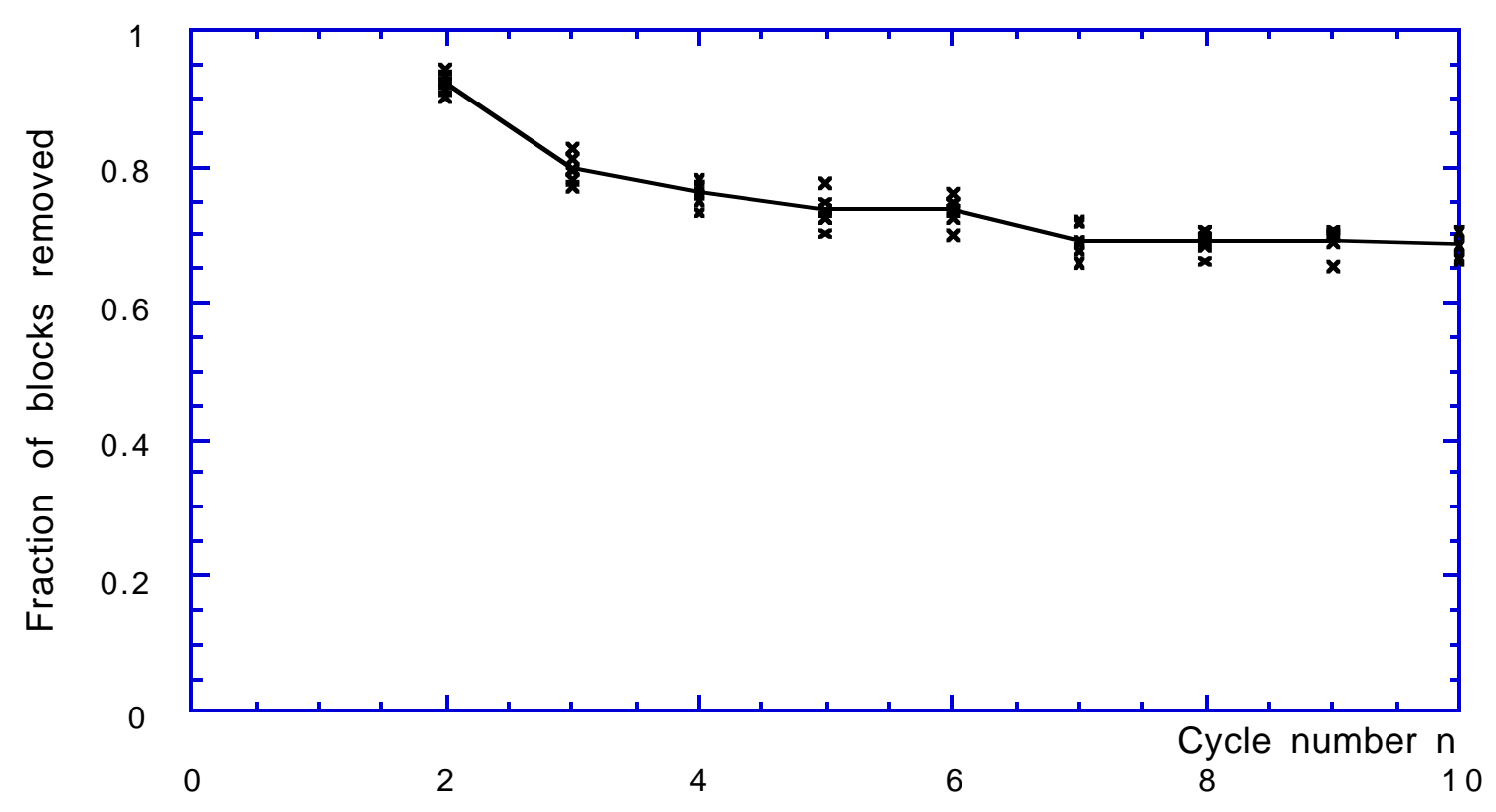

Figure 4.3b: Plot of Fraction of those blocks removed in cycle n, which are also removed in cycle $\mathrm{n}+1$ Vs. cycle number $\mathrm{n}$. for $\mathrm{T}=0.5$ and $P_{\max }=P_{\frac{1}{2}}-0.04$ for each cycle. 


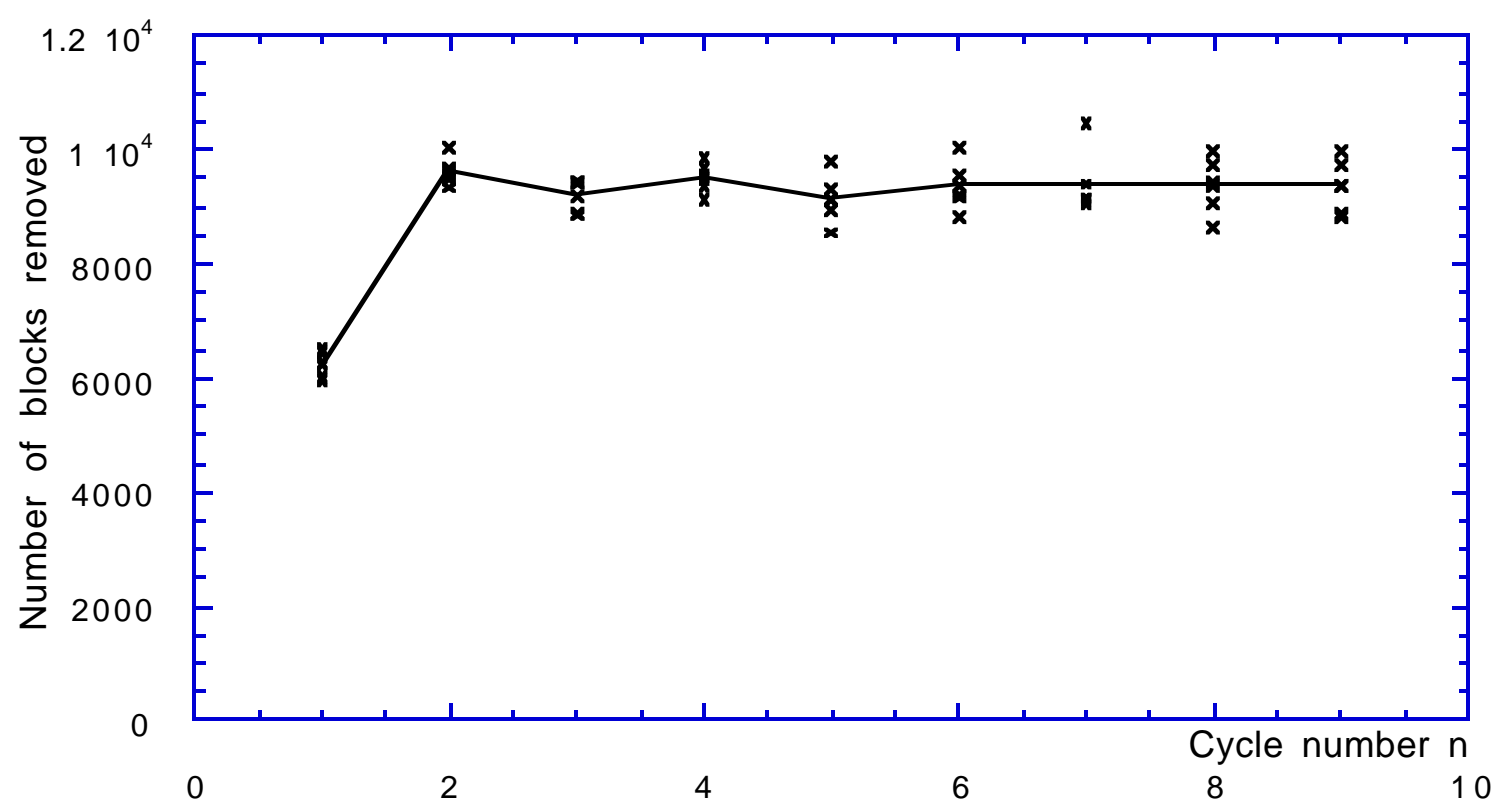

Figure 4.4a: Plot of the total number of blocks removed in cycle $n$ Vs. the cycle number $n$, for thickness parameter $\mathrm{T}=1.0$ and maximum pressure $P_{\max }=P_{\frac{1}{2}}+0.02$ for each cycle.

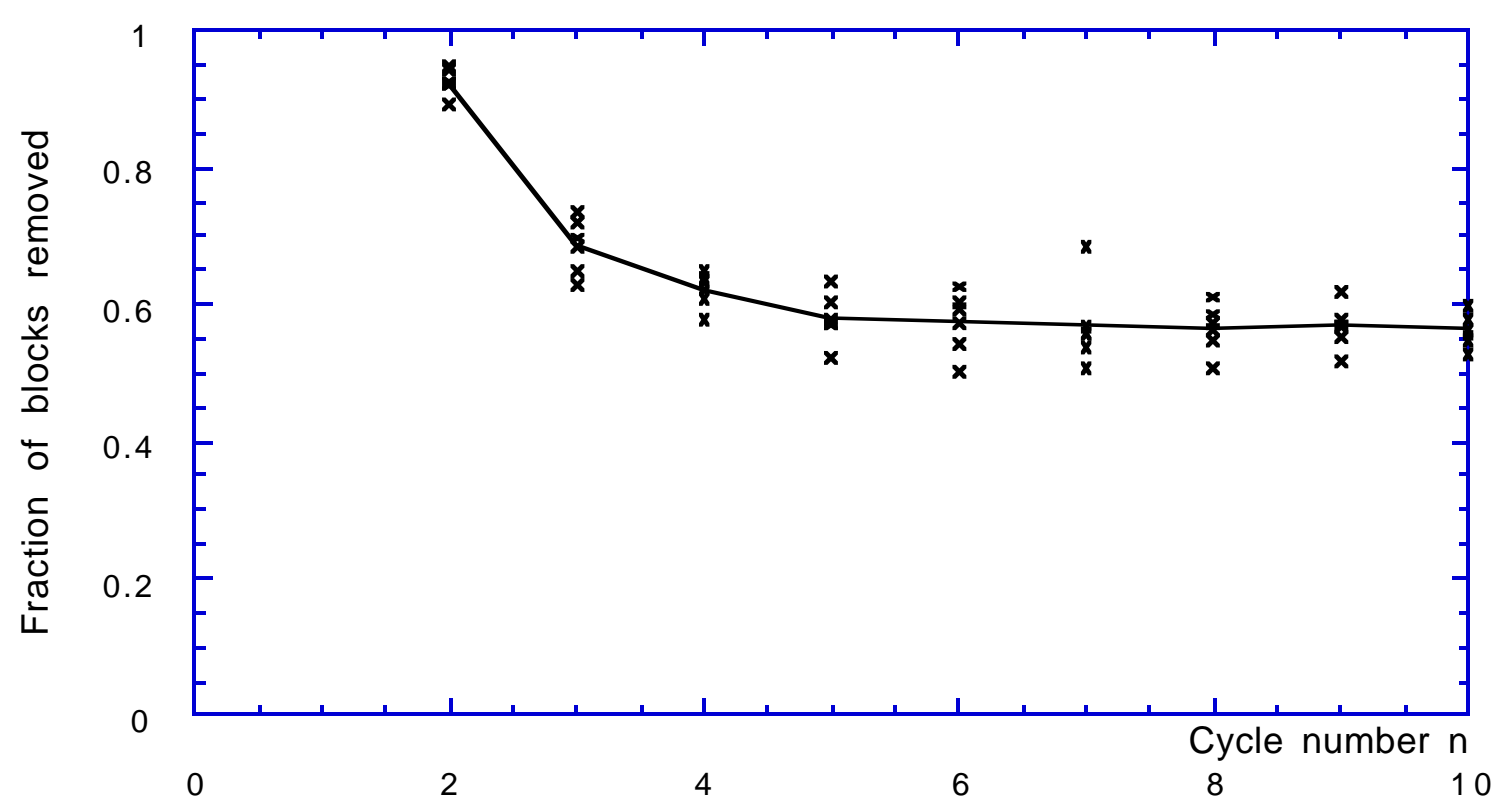

Figure 4.4b: Plot of Fraction of those blocks removed in cycle $n$, which are also removed in cycle $\mathrm{n}+1 \mathrm{Vs}$. cycle number $\mathrm{n}$, for $\mathrm{T}=1.0$ and $P_{\max }=P_{\frac{1}{2}}+0.02$ for each cycle. 


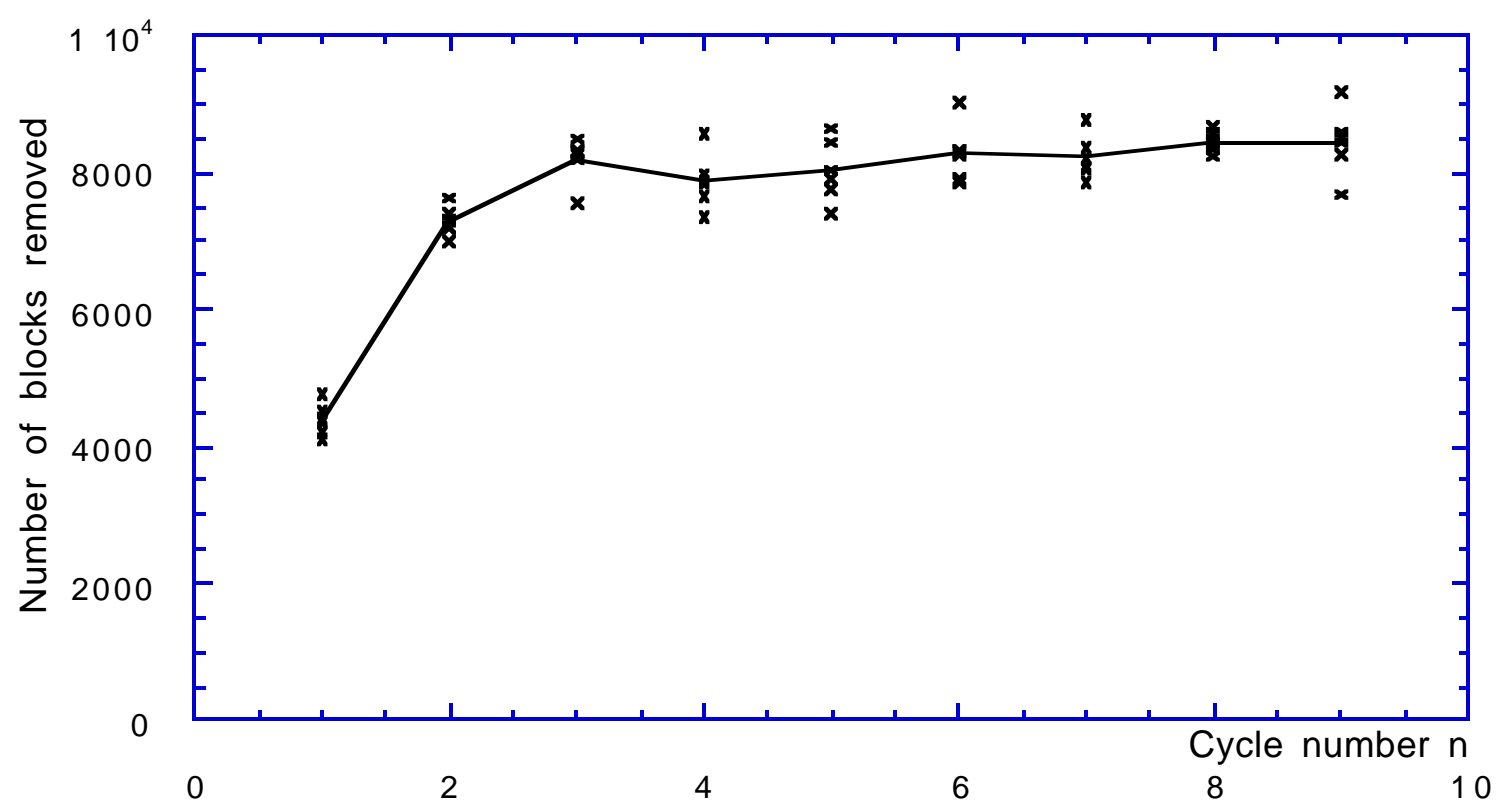

Figure 4.5a: Plot of the total number of blocks removed in cycle $n$ Vs. the cycle number $\mathrm{n}$, for thickness parameter T=1.0 and maximum pressure $P_{\max }=P_{\frac{1}{2}}+0.01$ for each cycle.

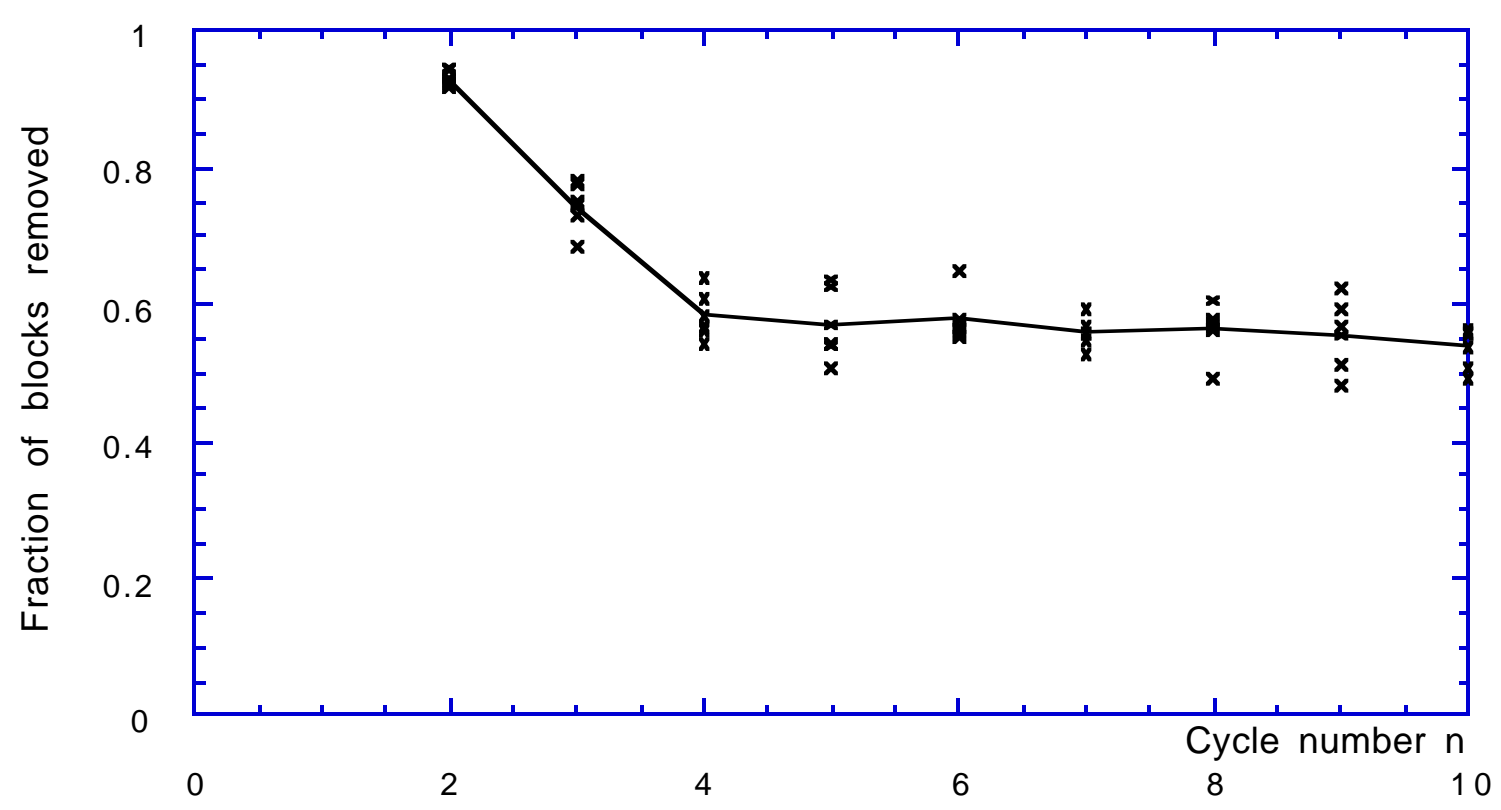

Figure 4.5b: Plot of Fraction of those blocks removed in cycle n, which are also removed in cycle $\mathrm{n}+1$ Vs. cycle number $\mathrm{n}$, for $\mathrm{T}=1.0$ and $P_{\max }=P_{\frac{1}{2}}+0.01$ for each cycle. 


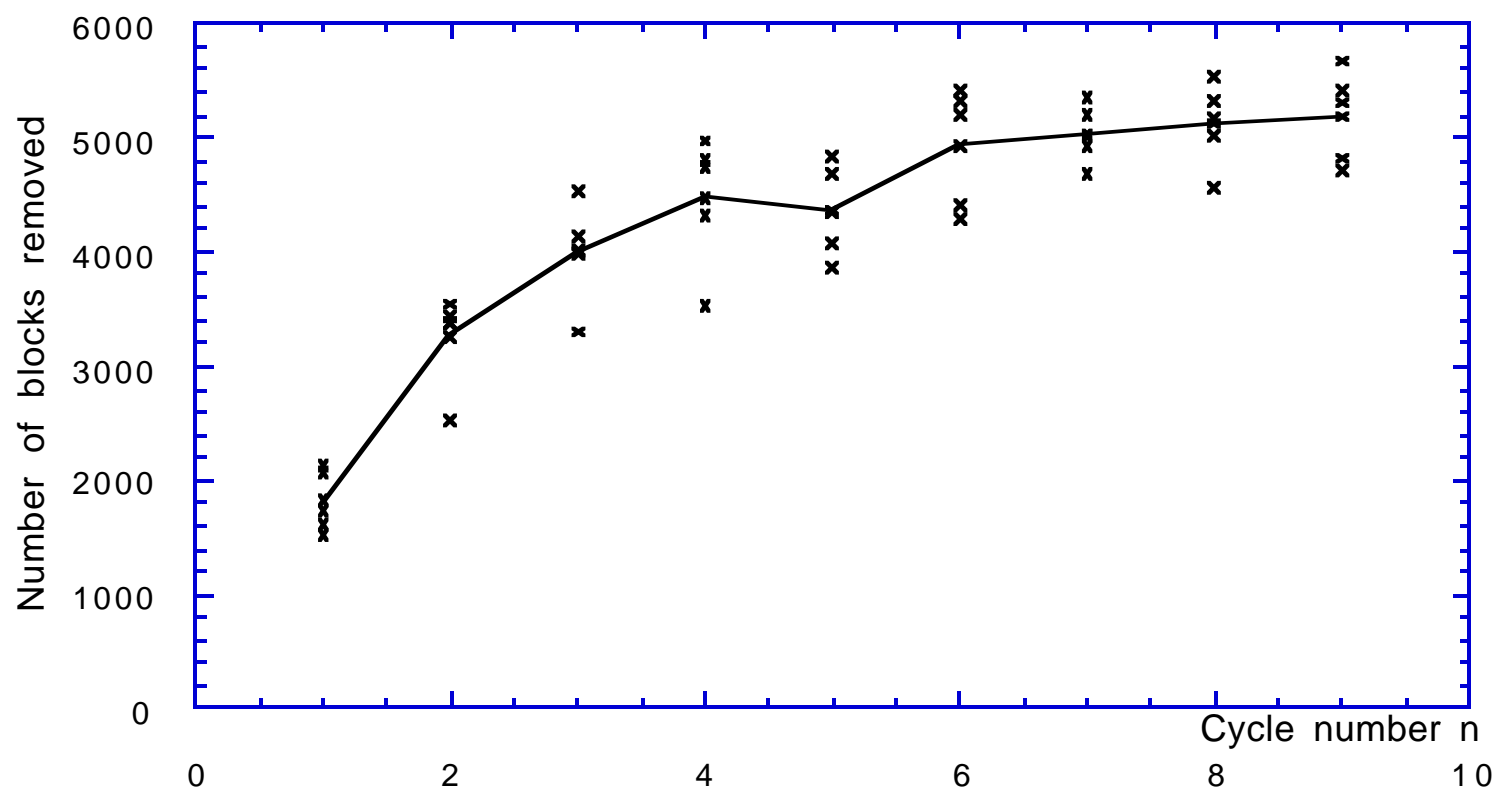

Figure 4.6a: Plot of the total number of blocks removed in cycle $n$ Vs. the cycle number n, for thickness parameter T=1.0 and maximum pressure $P_{\max }=P_{\frac{1}{2}}-0.01$ for each cycle.

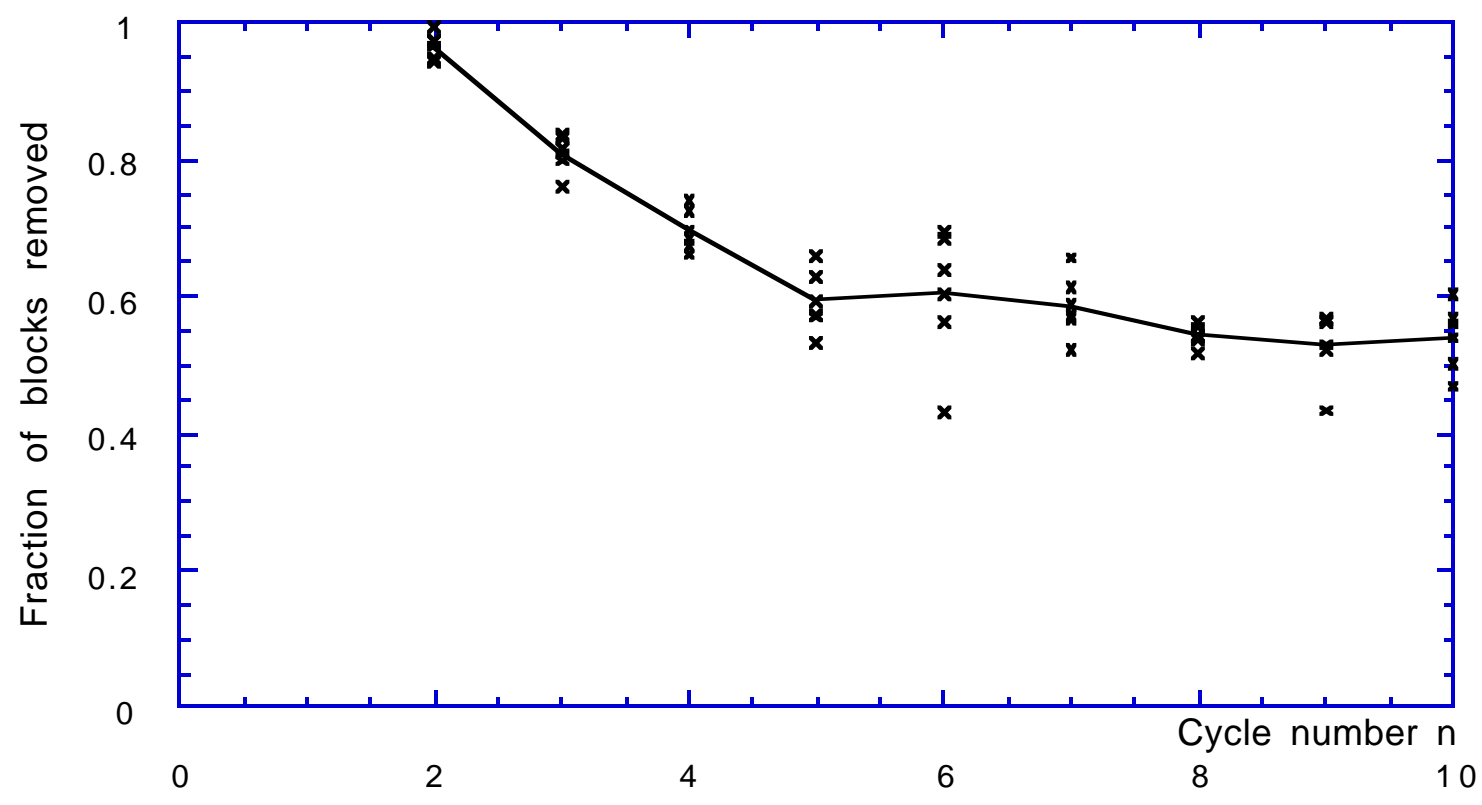

Figure 4.6b: Plot of Fraction of those blocks removed in cycle n, which are also removed in cycle $\mathrm{n}+1$ Vs. cycle number $\mathrm{n}$, for $\mathrm{T}=1.0$ and $P_{\max }=P_{\frac{1}{2}}-0.01$ for each cycle. 


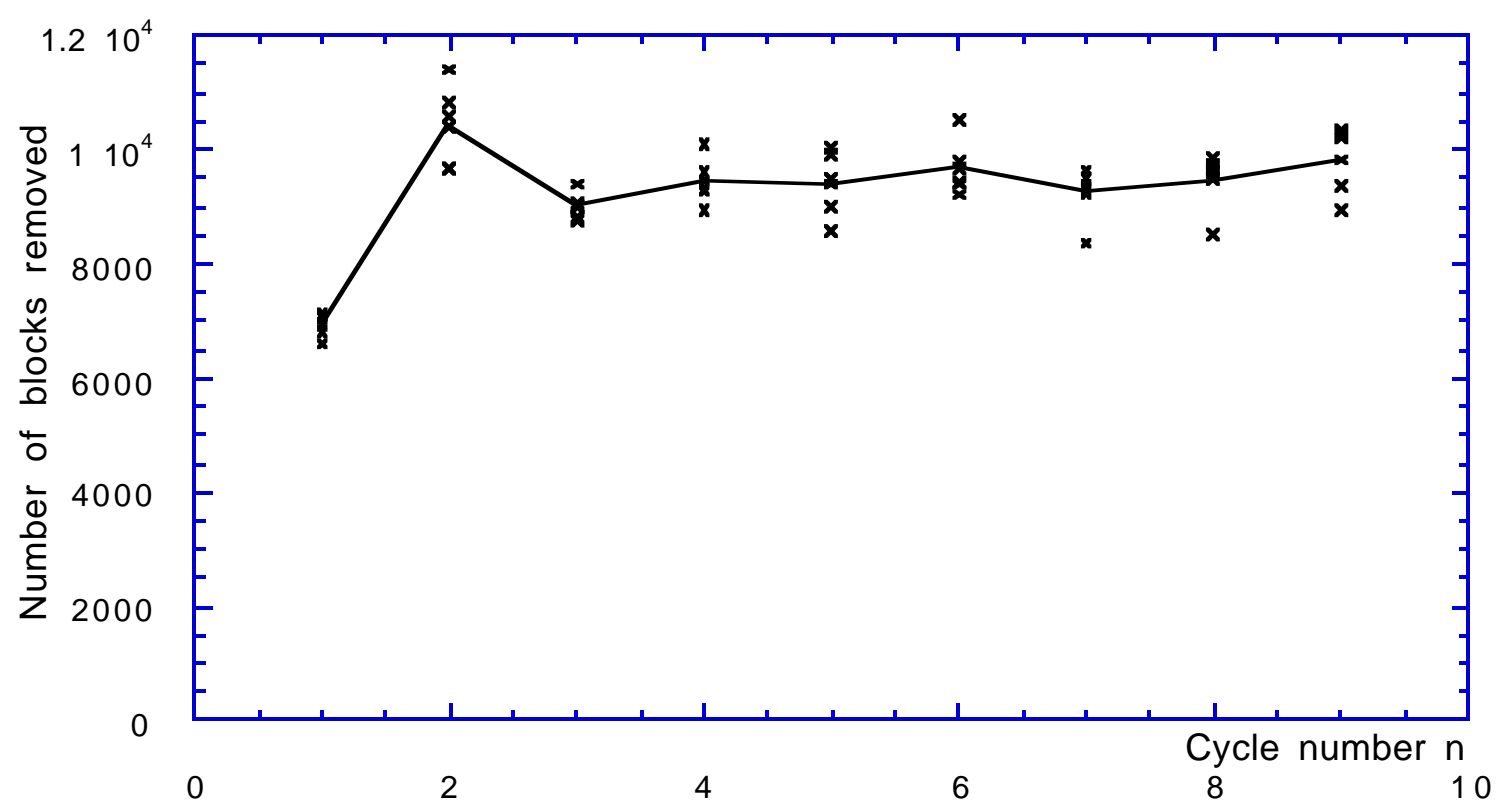

Figure 4.7a: Plot of the total number of blocks removed in cycle $n$ Vs. the cycle number $n$, for thickness parameter $\mathrm{T}=1.5$ and maximum pressure $P_{\max }=P_{\frac{1}{2}}+0.03$ for each cycle.

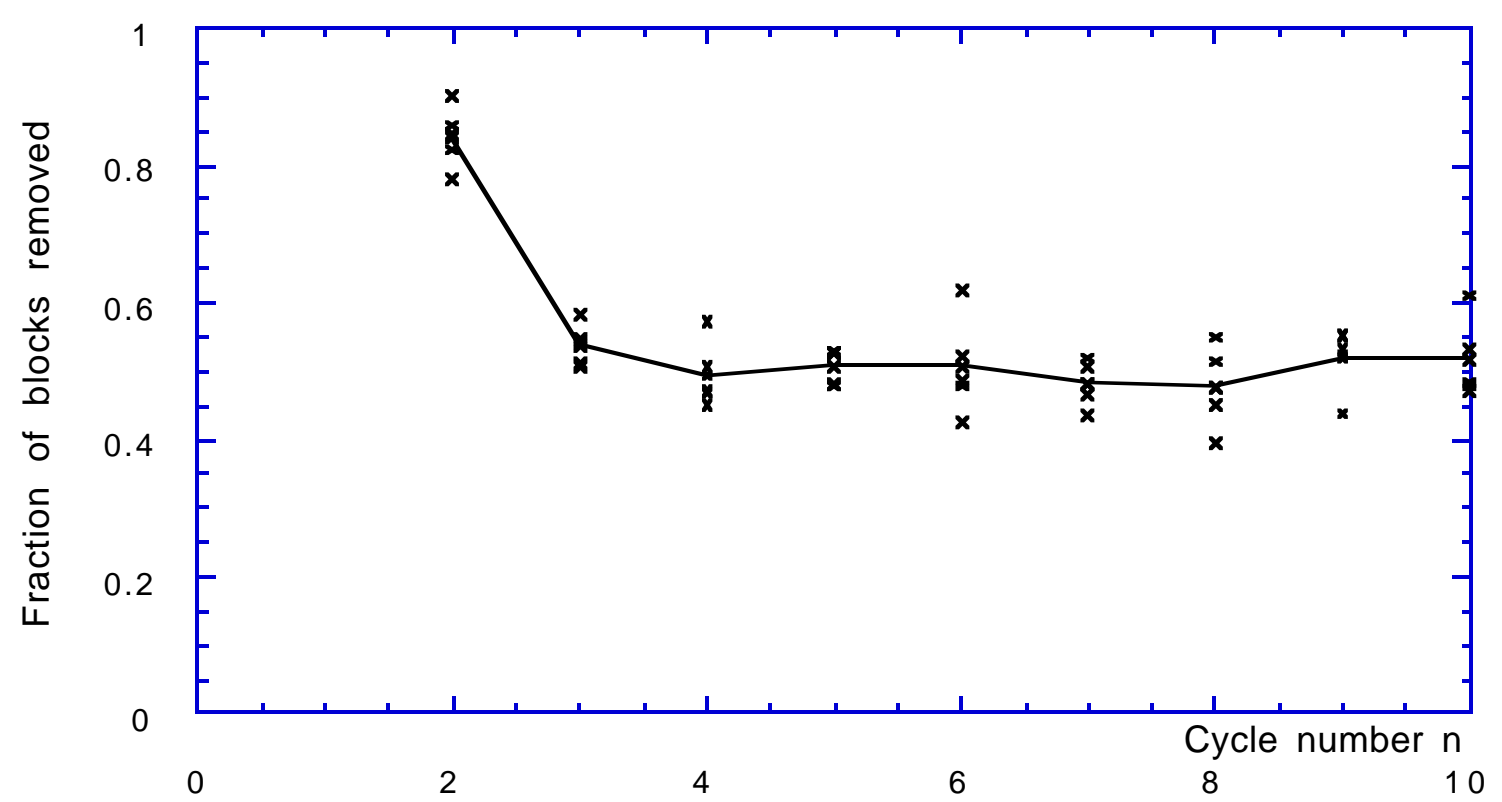

Figure 4.7b: Plot of Fraction of those blocks removed in cycle n, which are also removed in cycle $\mathrm{n}+1 \mathrm{Vs}$. cycle number $\mathrm{n}$, for $\mathrm{T}=1.5$ and $P_{\max }=P_{1}+0.03$ for each cycle. 


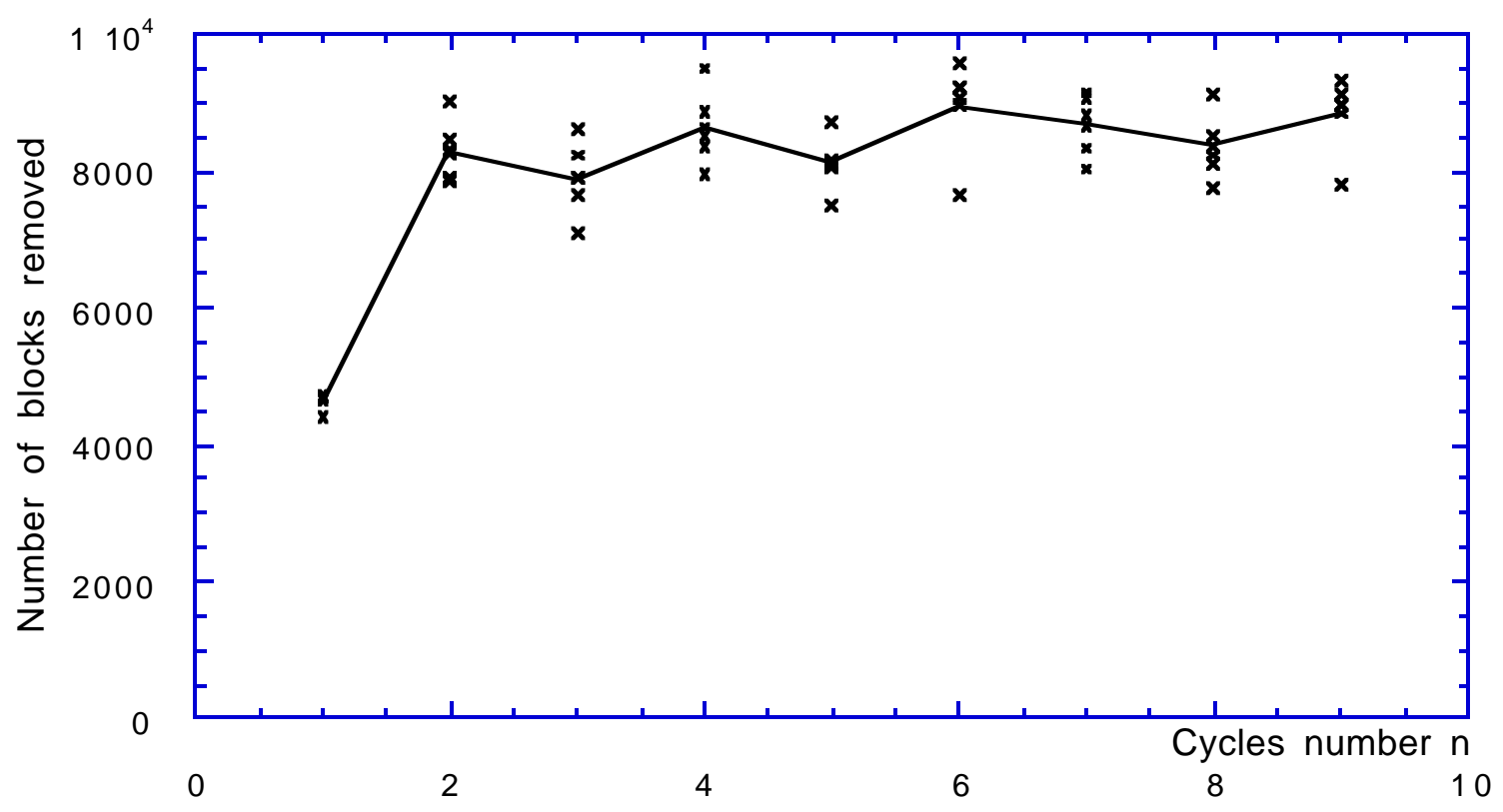

Figure 4.8a: Plot of the total number of blocks removed in cycle $n$ Vs. the cycle number $n$, for thickness parameter $\mathrm{T}=1.5$ and maximum pressure $P_{\max }=P_{\frac{1}{2}}+0.02$ for each cycle.

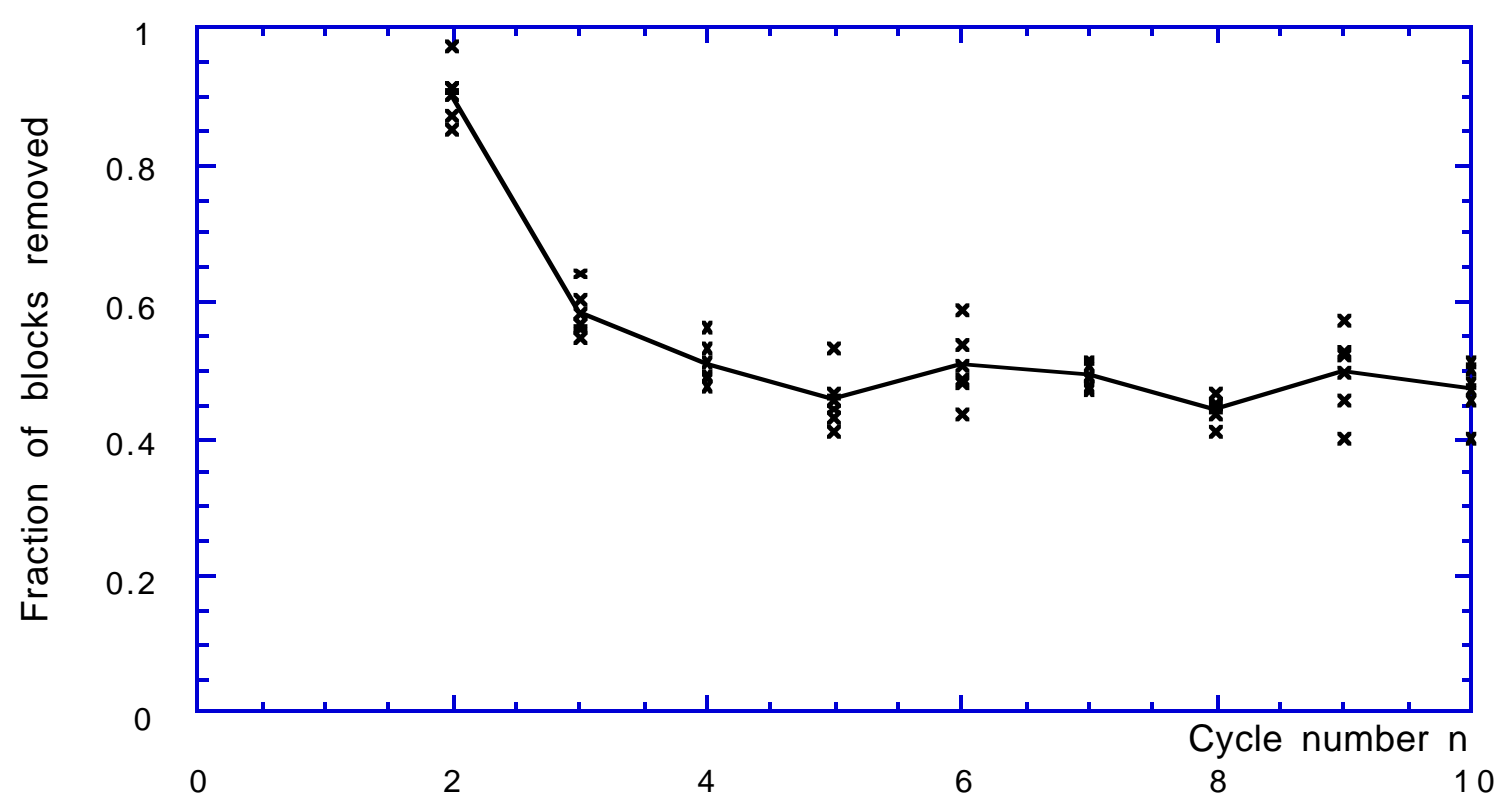

Figure 4.8b: Plot of Fraction of those blocks removed in cycle $\mathrm{n}$, which are also removed in cycle $\mathrm{n}+1 \mathrm{Vs}$. cycle number $\mathrm{n}$, for $\mathrm{T}=1.5$ and $P_{\max }=P_{\frac{1}{2}}+0.02$ for each cycle. 


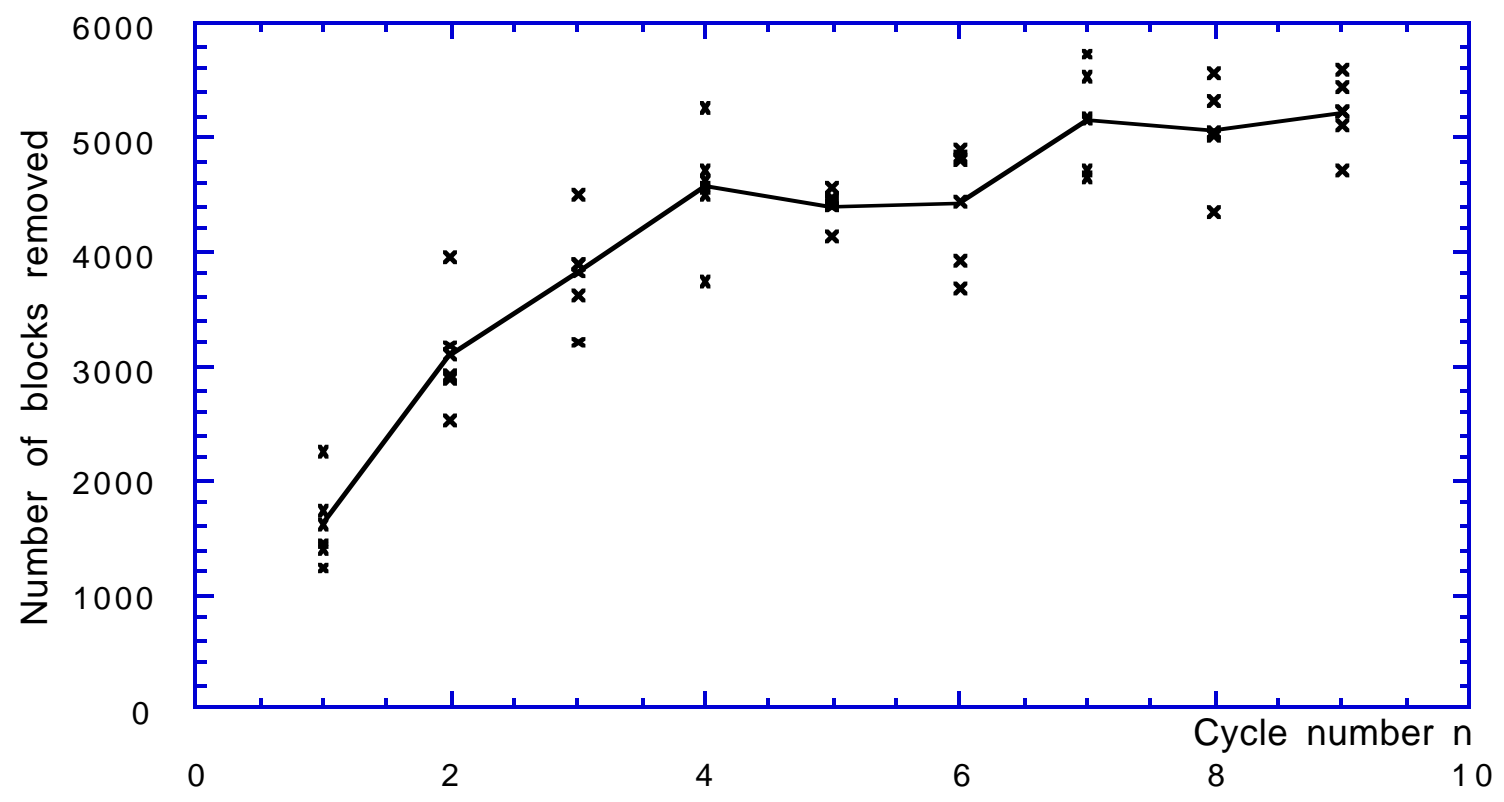

Figure 4.9a: Plot of the total number of blocks removed in cycle $n$ Vs. the cycle number $n$, for thickness parameter $\mathrm{T}=1.5$ and maximum pressure $P_{\max }=P_{\frac{1}{2}}+0.0$ for each cycle.

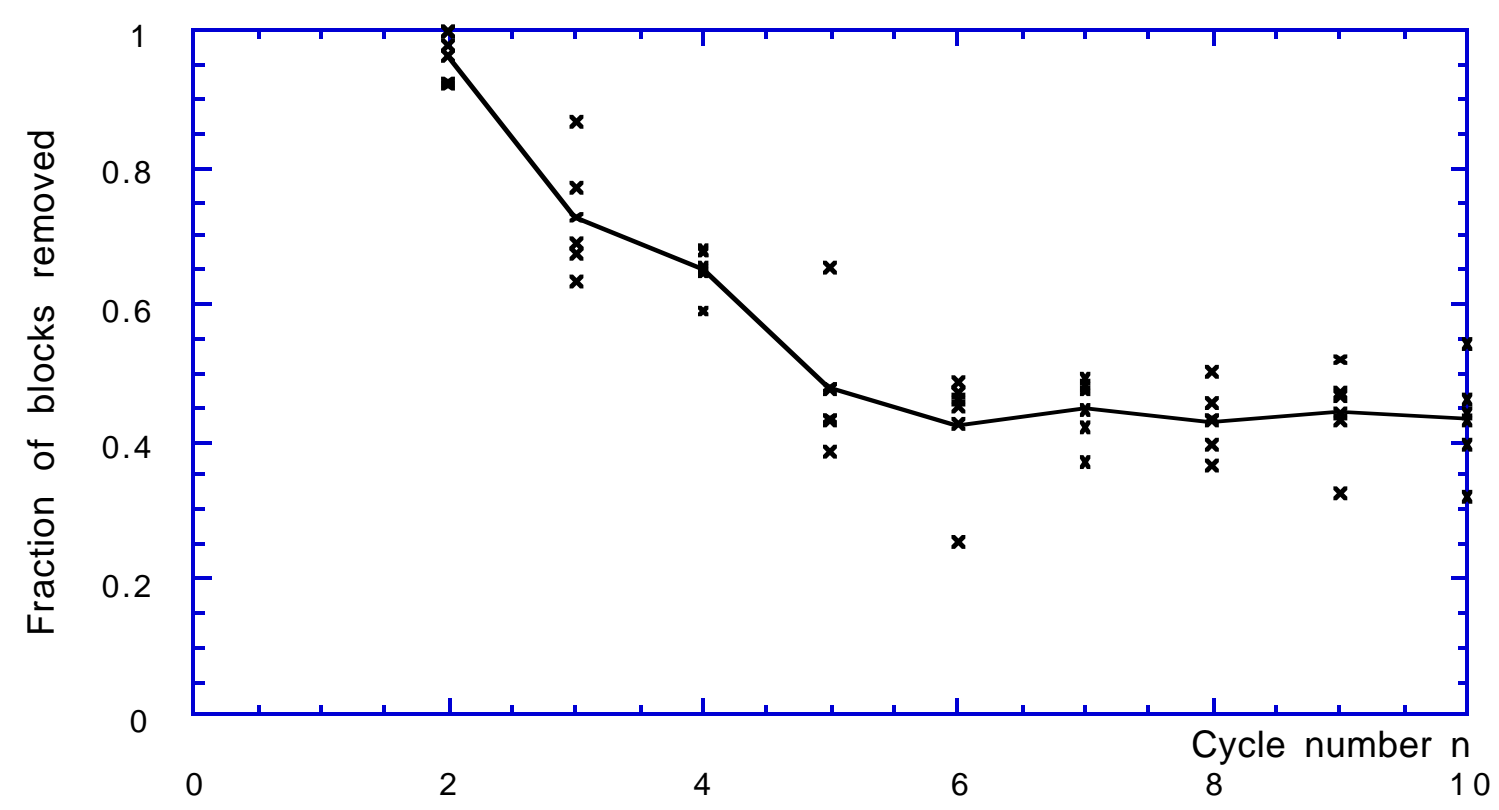

Figure 4.9b: Plot of Fraction of those blocks removed in cycle n, which are also removed in cycle $\mathrm{n}+1$ Vs. cycle number $\mathrm{n}$, for $\mathrm{T}=1.5$ and $P_{\max }=P_{\frac{1}{2}}+0.0$ for each cycle. 


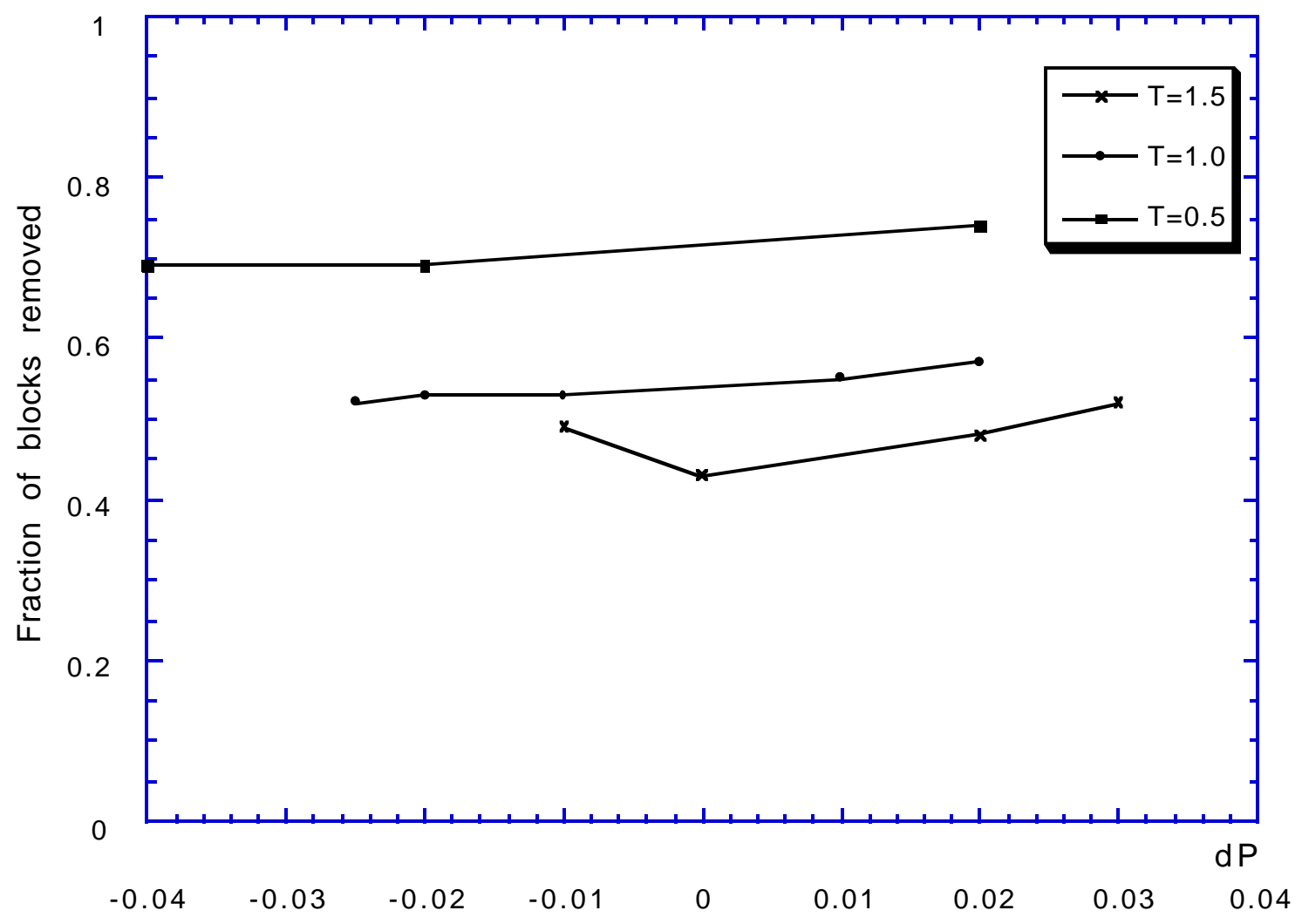

Figure 4.10: A plot summarizing Figures 4.1b-4.9b, showing trends in the fraction of blocks that are removed in two successive cycles, as an average over the last few cycles Vs. differential pressure $\mathrm{dP}$, for the three thickness parameters $\mathrm{T}$.

\subsection{Discussion of trends in Figure 4.10}

The results in the Figures $4.1 \mathrm{~b}-4.9 \mathrm{~b}$ are summarized in Figure 4.10. The figure show the fraction of blocks removed in two successive cycles and averaged over the last few cycles plotted against the differential pressure $\mathrm{dP}$. The plot shows that as the thickness parameter increases, the fraction of those blocks removed in the cycle $\mathrm{n}$ which is also removed in cycle $n+1$ decreases. Also for each thickness parameters there is little variation with differential pressure in the fraction of blocks that are removed in 2 successive cycles. 


\subsection{Blocks which are lifted in one cycle and removed in the next.}

The computer program also calculates the total number of blocks that are lifted (but not

removed) in each cycle. It plots this number against the cycle number n, in Figures 4.11a4.19a. In addition the program calculates the number of blocks which are lifted in cycle $n$ and subsequently removed in cycle $\mathrm{n}+1$, as a fraction of the total number of blocks removed in cycle $n+1$, and plots this against cycle number $n$ in Figures $4.11 b-4.19 b$. To facilitate observing the trends in Figures 4.11b-4.19b, their results are summarized in Figure 4.20 which plots the fraction of blocks lifted in one cycle and removed in the next (averaged over the last few cycles).

The Figures 4.11-4.20 are as shown on the following pages. 


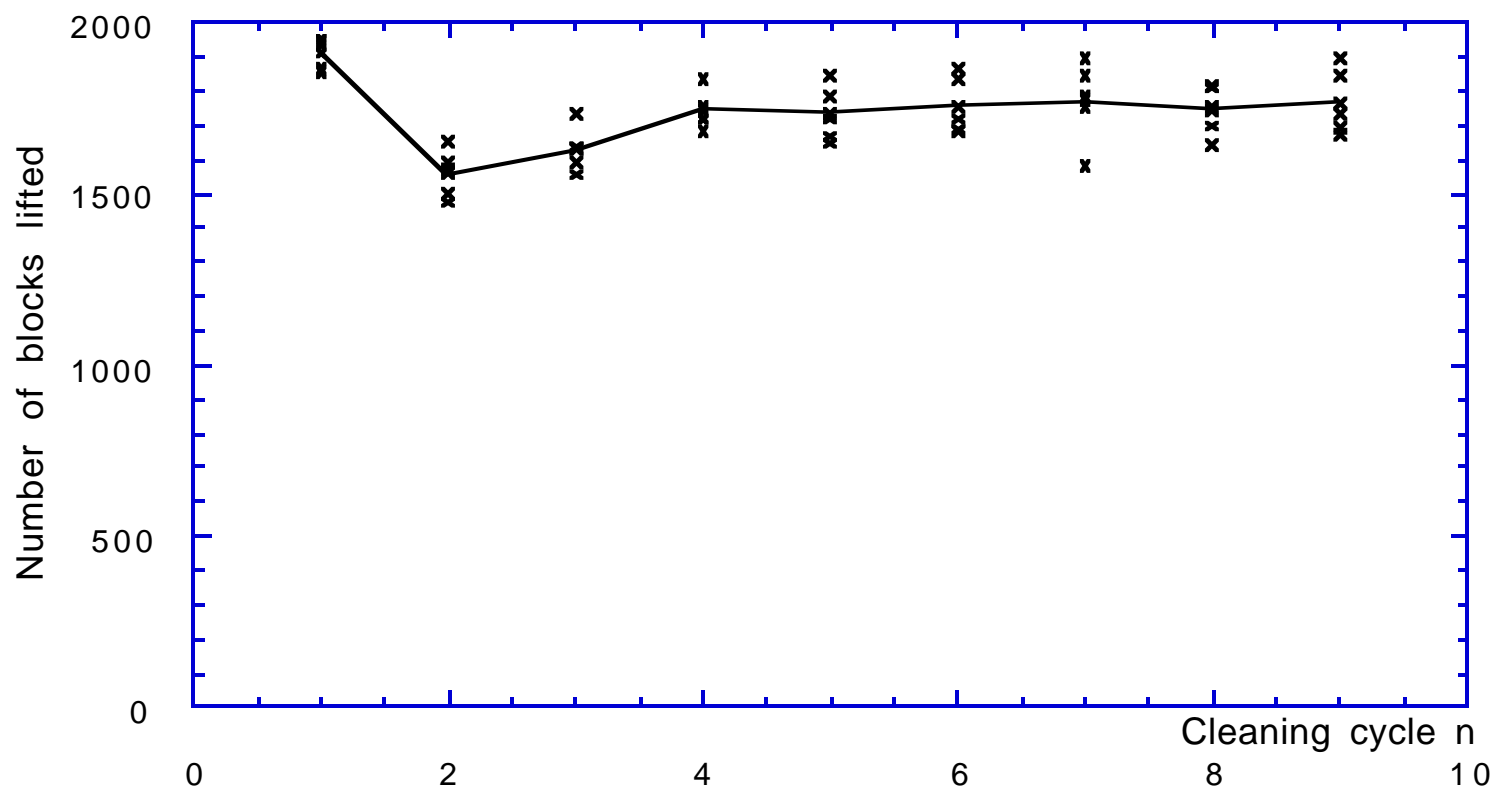

Figure 4.11a: Plot of total number of blocks lifted in cycle n Vs. the cycle number n, for thickness parameter T=0.5 and maximum pressure $P_{\max }=P_{\frac{1}{2}}+0.02$ for each cycle.

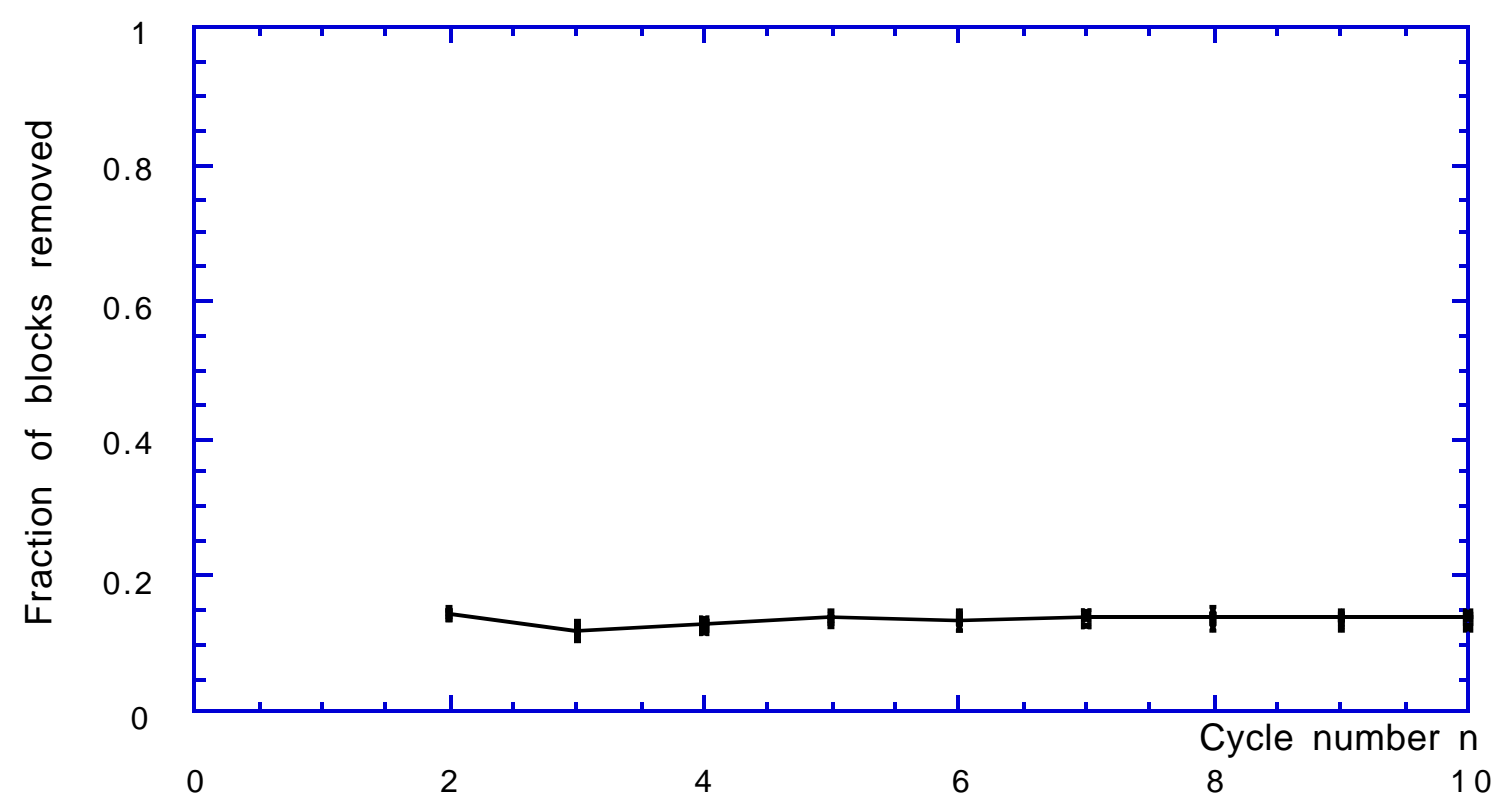

Figure 4.11b: Plot of the number of blocks lifted in cycle $n$ and removed in cycle $n+1$, (as a fraction of the total number of blocks removed in cycle $n+1$ ) Vs. the cycle number $n$, for thickness parameter $\mathrm{T}=0.5$ and maximum pressure $P_{\max }=P_{1}+0.02$ for each cycle. 


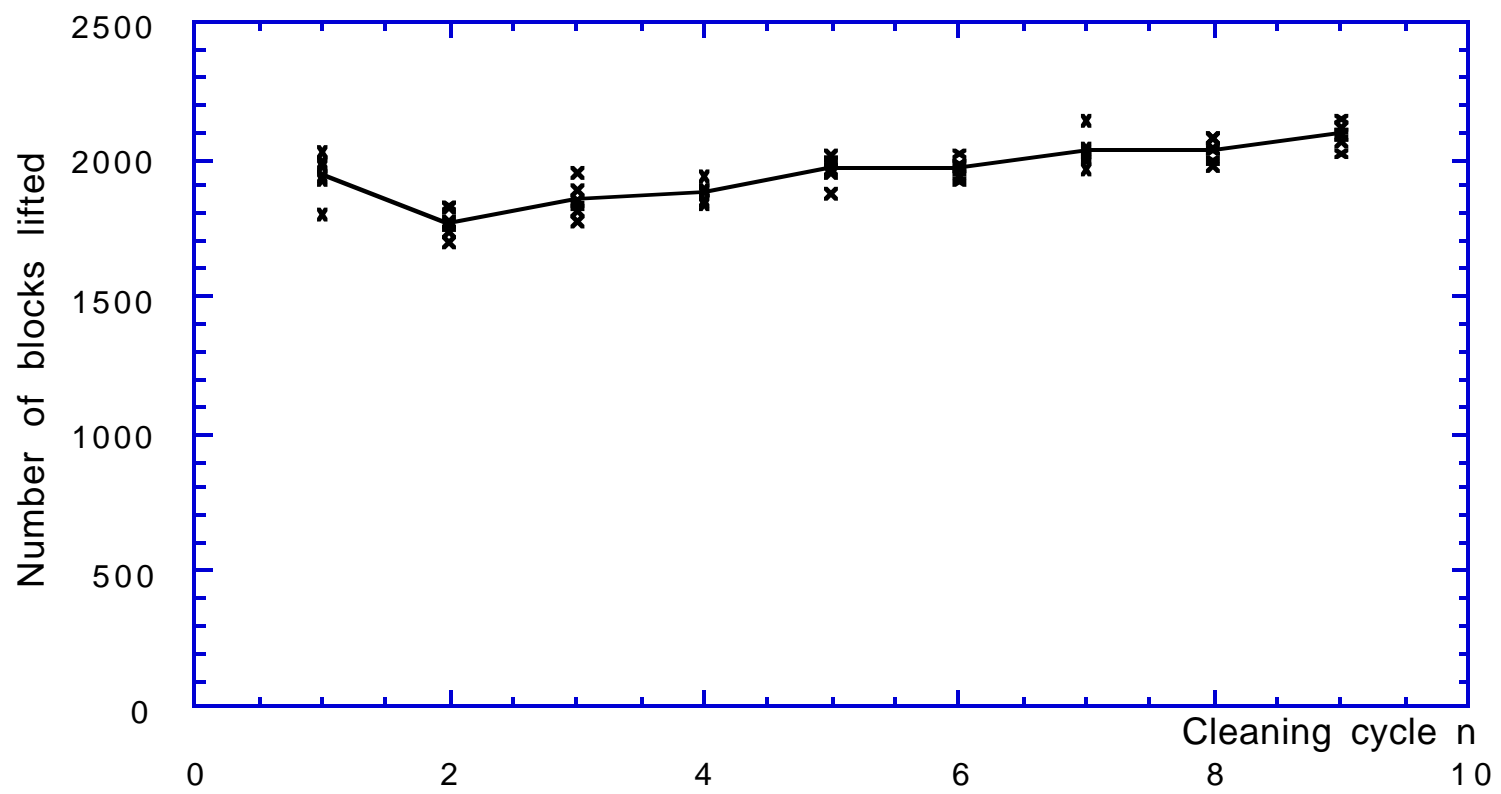

Figure 4.12a: Plot of total number of blocks lifted in cycle n Vs. the cycle number n, for thickness parameter $\mathrm{T}=0.5$ and maximum pressure $P_{\max }=P_{\frac{1}{2}}-0.02$ for each cycle.

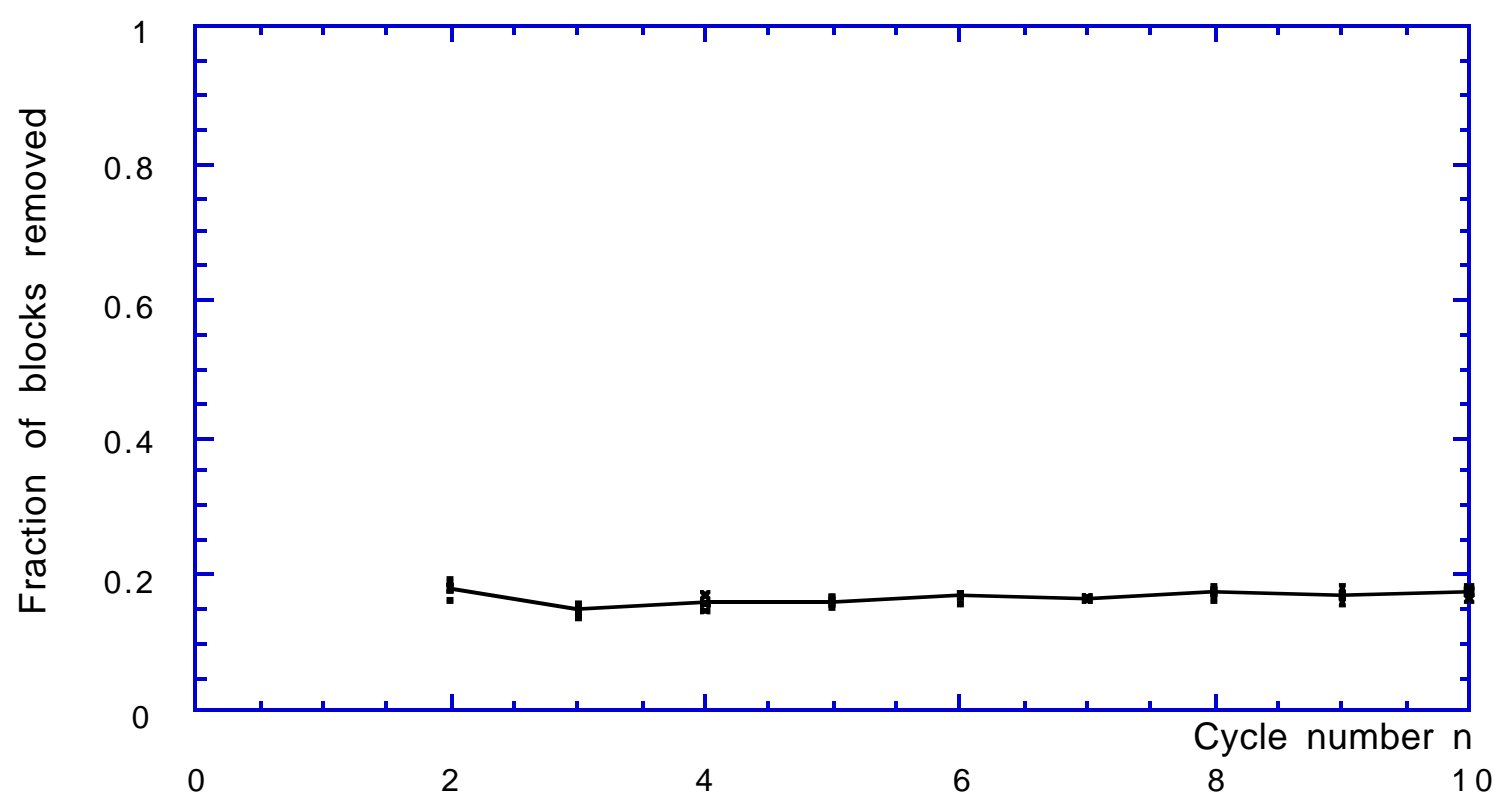

Figure 4.12b: Plot of the number of blocks lifted in cycle $n$ and removed in cycle $n+1$, (as a fraction of the total number of blocks removed in cycle $n+1$ ) Vs. the cycle number $n$, for thickness parameter $\mathrm{T}=0.5$ and maximum pressure $P_{\max }=P_{\frac{1}{2}}-0.02$ for each cycle. 


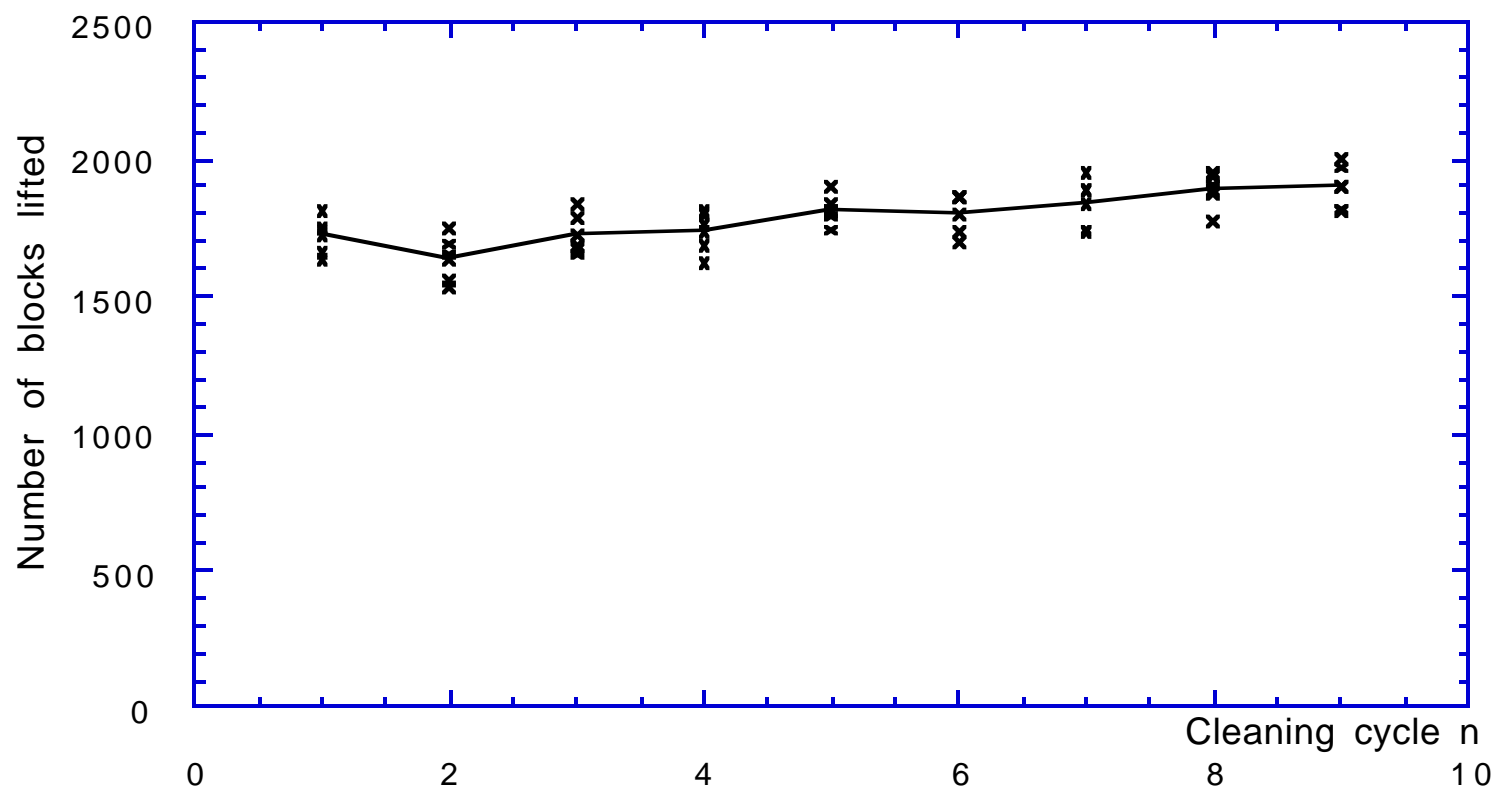

Figure 4.13a: Plot of total number of blocks lifted in cycle n Vs. the cycle number n, for thickness parameter $\mathrm{T}=0.5$ and maximum pressure $P_{\max }=P_{\frac{1}{2}}-0.04$ for each cycle.

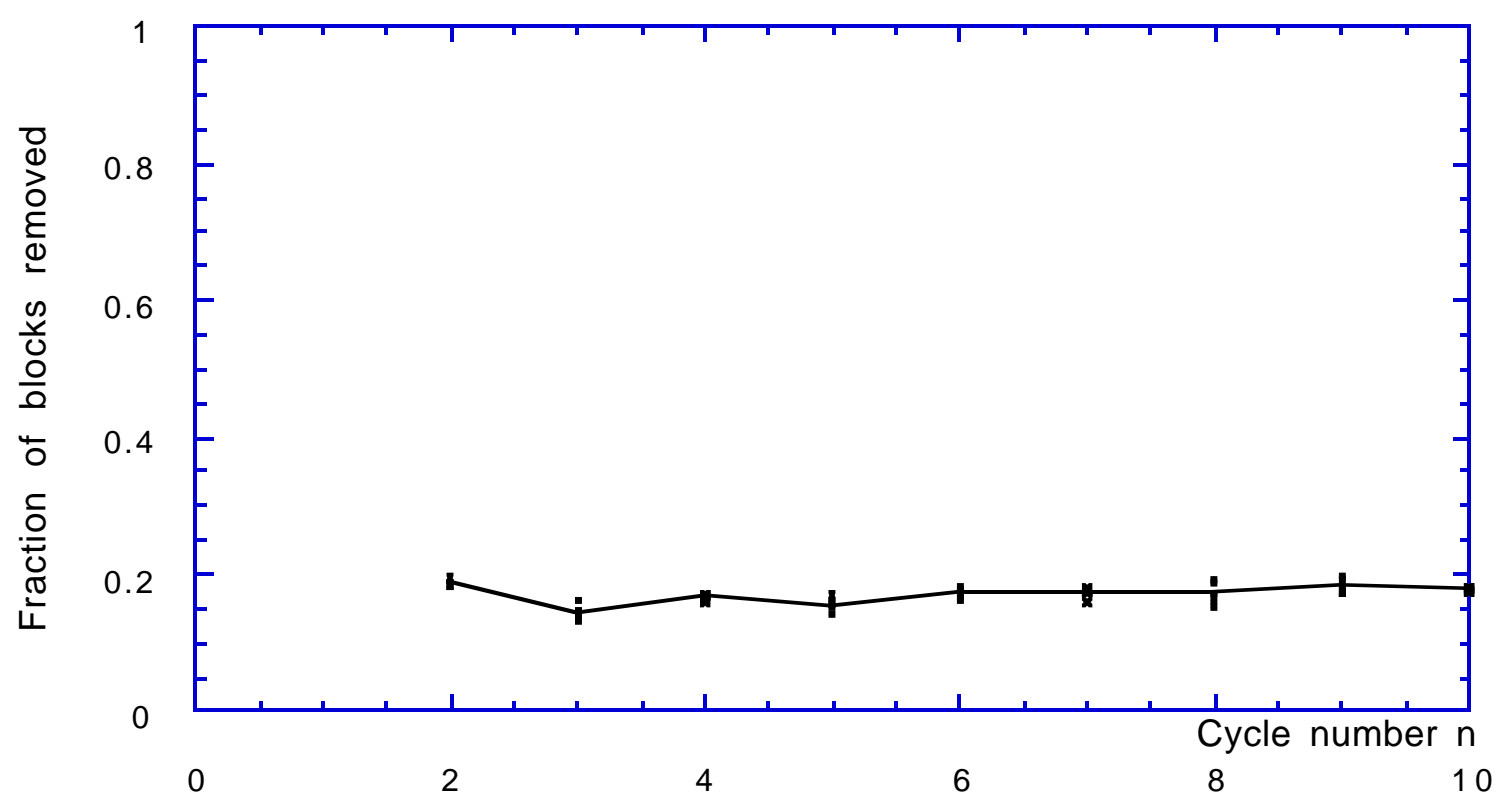

Figure 4.13b: Plot of the number of blocks lifted in cycle $n$ and removed in cycle $n+1$, (as a fraction of the total number of blocks removed in cycle $n+1$ ) Vs. the cycle number $n$, for thickness parameter $\mathrm{T}=0.5$ and maximum pressure $P_{\max }=P_{\frac{1}{2}}-0.04$ for each cycle. 


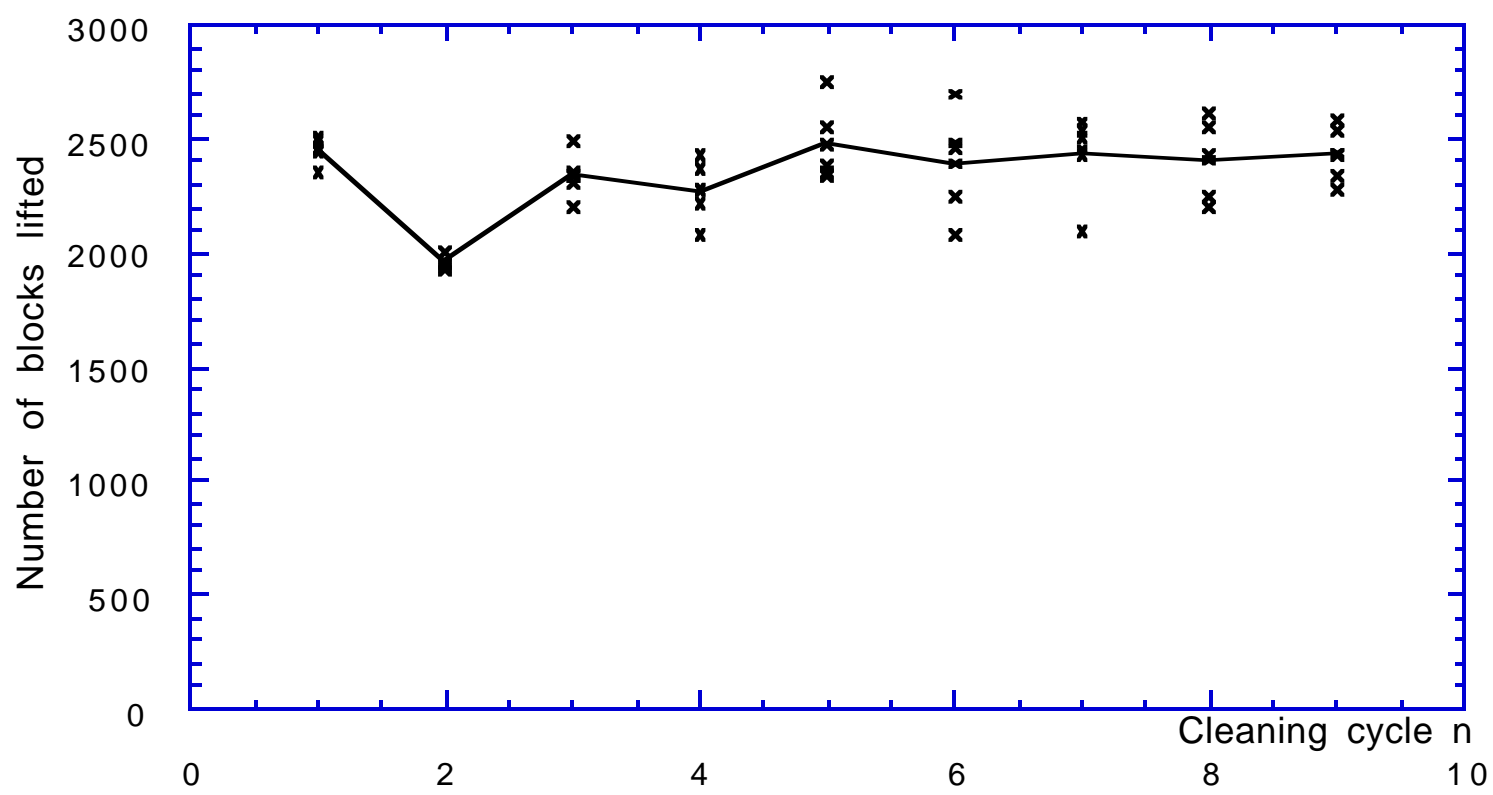

Figure 4.14a: Plot of total number of blocks lifted in cycle $n$ Vs. the cycle number n, for thickness parameter $\mathrm{T}=1.0$ and maximum pressure $P_{\max }=P_{\frac{1}{2}}+0.02$ for each cycle.

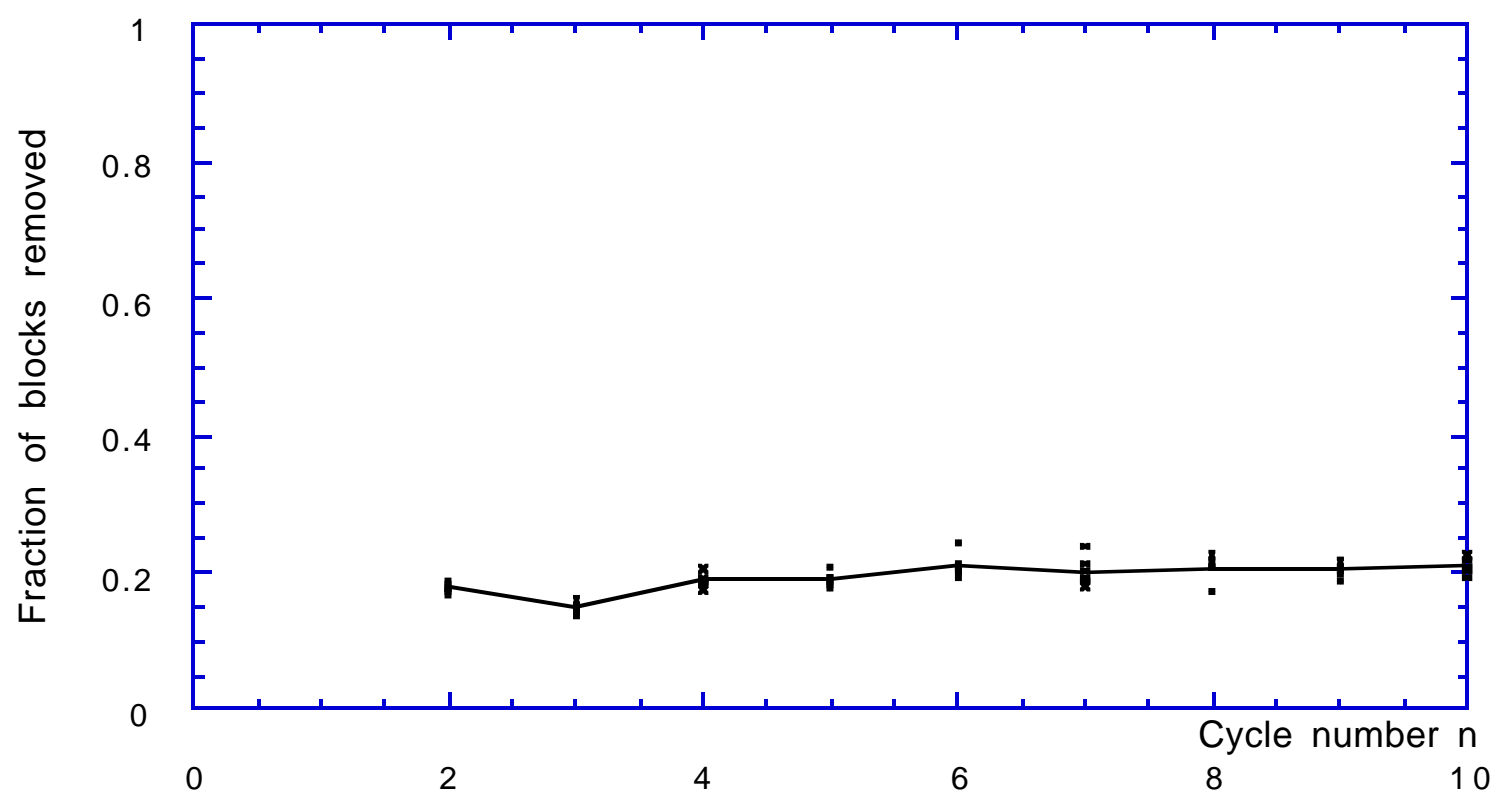

Figure 4.14b: Plot of the number of blocks lifted in cycle $n$ and removed in cycle $n+1$, (as a fraction of the total number of blocks removed in cycle $n+1$ ) Vs. the cycle number $n$, for thickness parameter $\mathrm{T}=1.0$ and maximum pressure $P_{\max }=P_{1}+0.02$ for each cycle. 


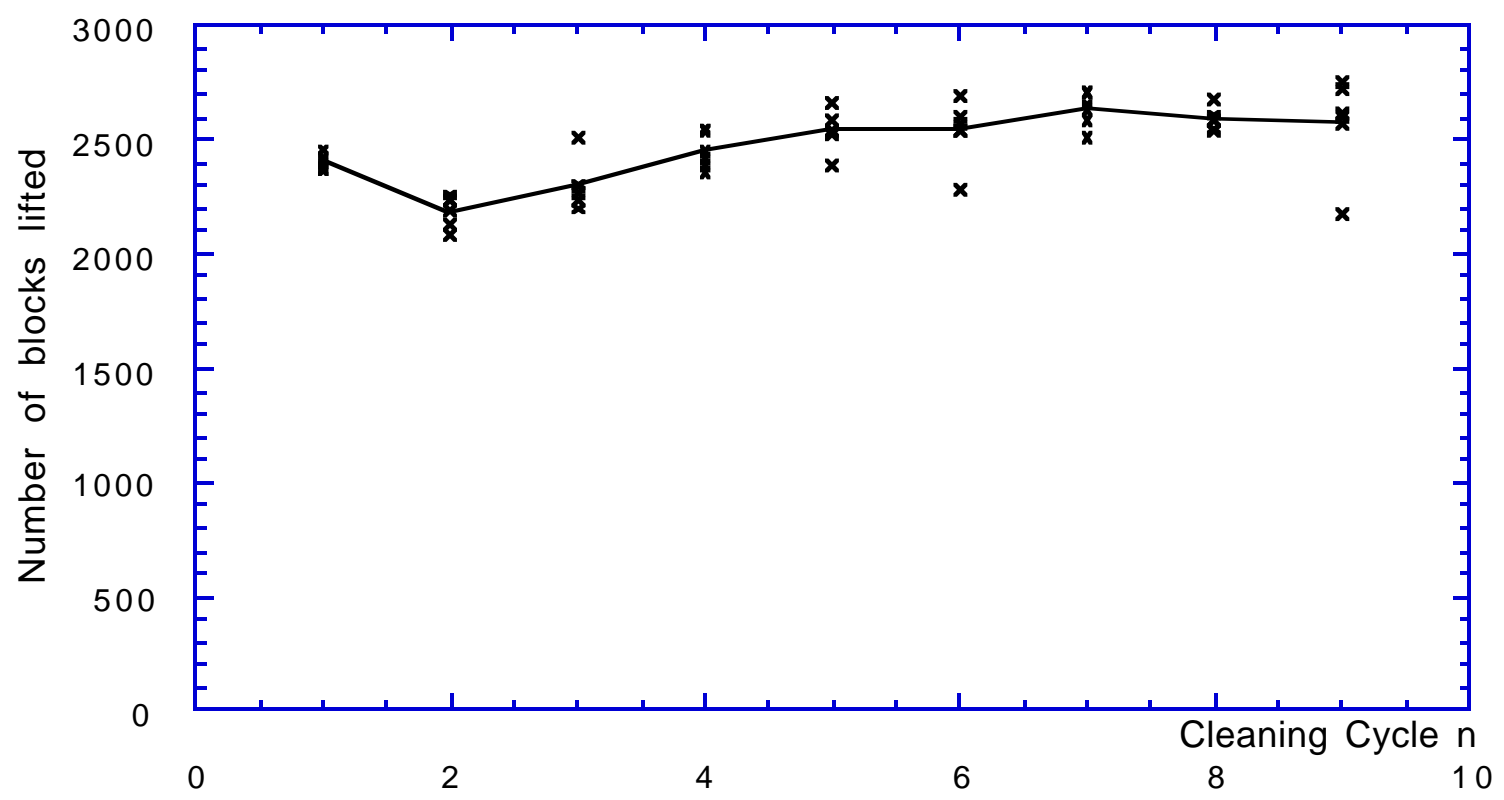

Figure 4.15a: Plot of total number of blocks lifted in cycle $n$ Vs. the cycle number $n$, for thickness parameter T=1.0 and maximum pressure $P_{\max }=P_{\frac{1}{2}}+0.01$ for each cycle.

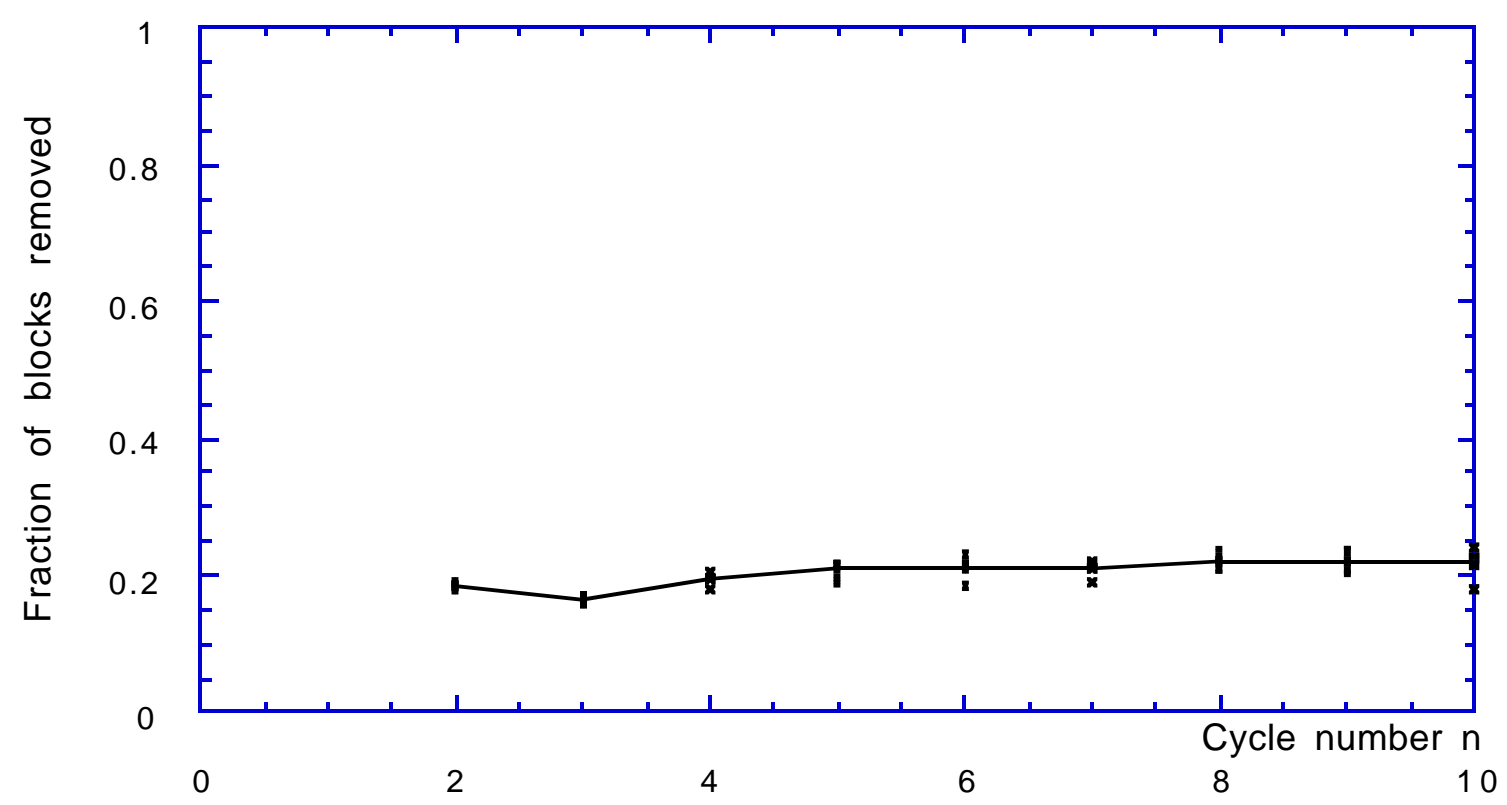

Figure 4.15b: Plot of the number of blocks lifted in cycle $n$ and removed in cycle $n+1$, (as a fraction of the total number of blocks removed in cycle $n+1$ ) Vs. the cycle number $n$, for thickness parameter $\mathrm{T}=1.0$ and maximum pressure $P_{\max }=P_{\frac{1}{2}}+0.01$ for each cycle. 


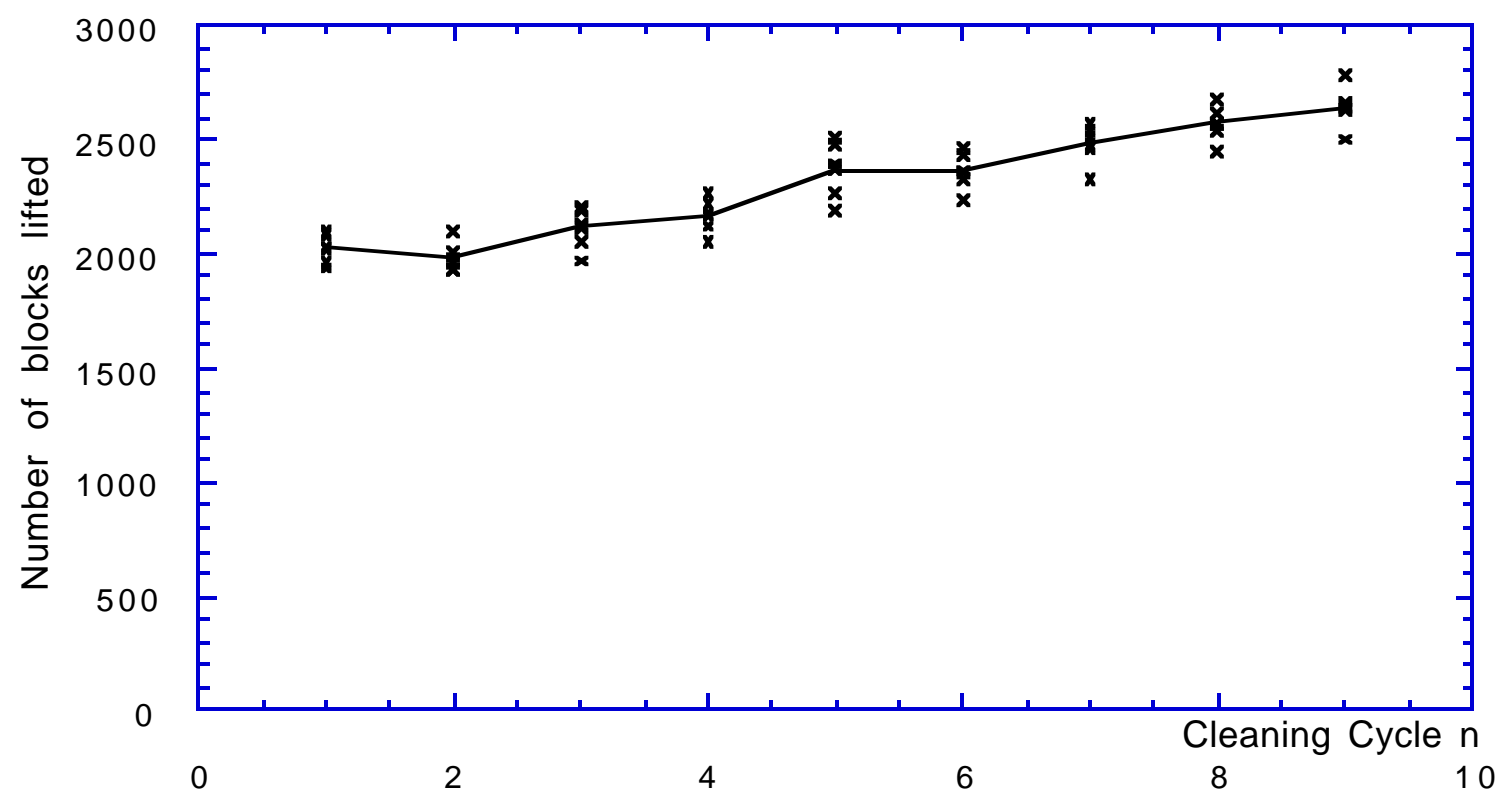

Figure 4.16a: Plot of total number of blocks lifted in cycle n Vs. the cycle number n, for thickness parameter T=1.0 and maximum pressure $P_{\max }=P_{\frac{1}{2}}-0.01$ for each cycle.

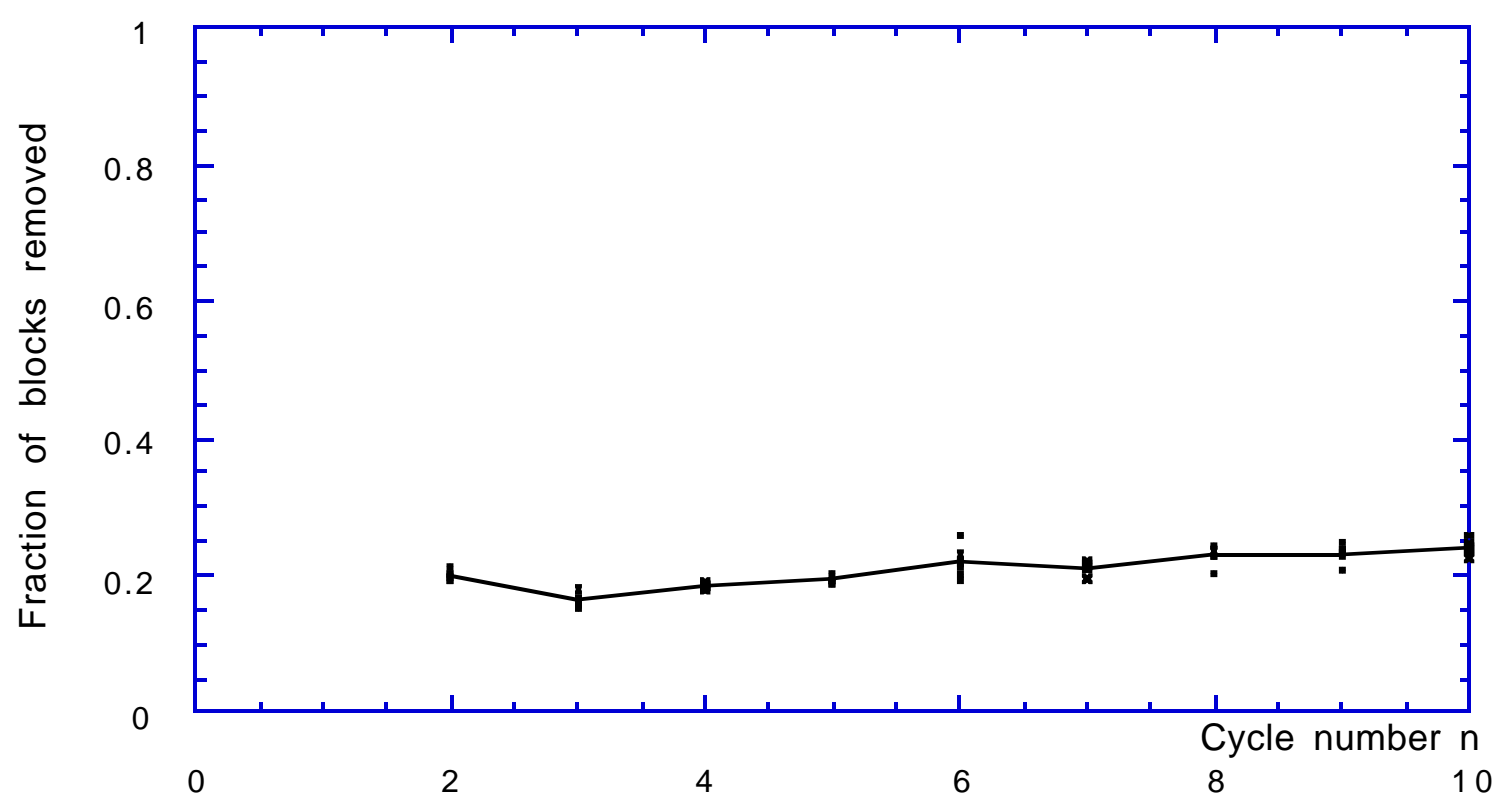

Figure 4.16b: Plot of the number of blocks lifted in cycle $\mathrm{n}$ and removed in cycle $\mathrm{n}+1$, (as a fraction of the total number of blocks removed in cycle $n+1$ ) Vs. the cycle number $n$, for thickness parameter $\mathrm{T}=1.0$ and maximum pressure $P_{\max }=P_{\frac{1}{2}}-0.01$ for each cycle. 


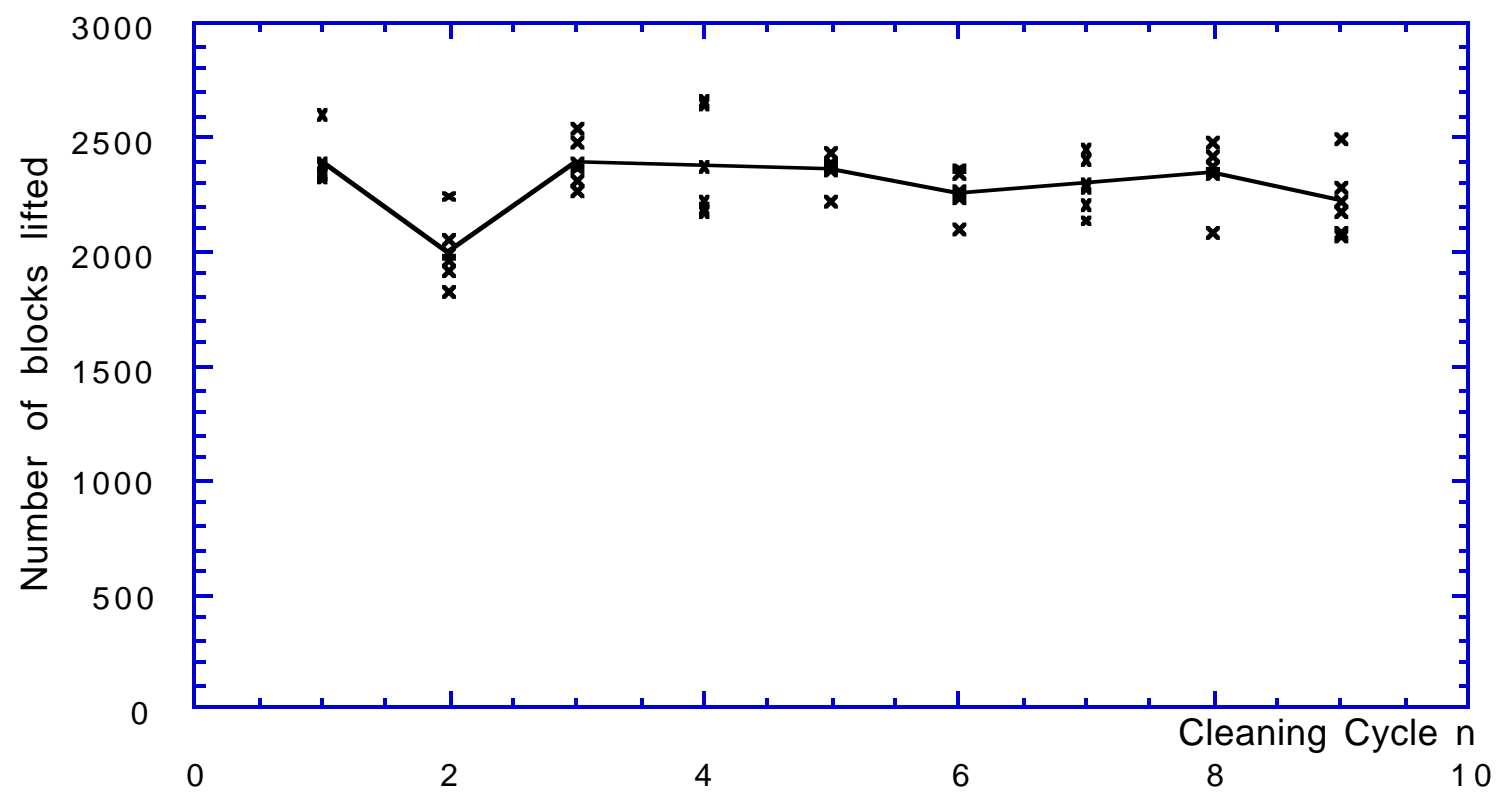

Figure 4.17a: Plot of total number of blocks lifted in cycle n Vs. the cycle number n, for thickness parameter T=1.5 and maximum pressure $P_{\max }=P_{\frac{1}{2}}+0.03$ for each cycle.

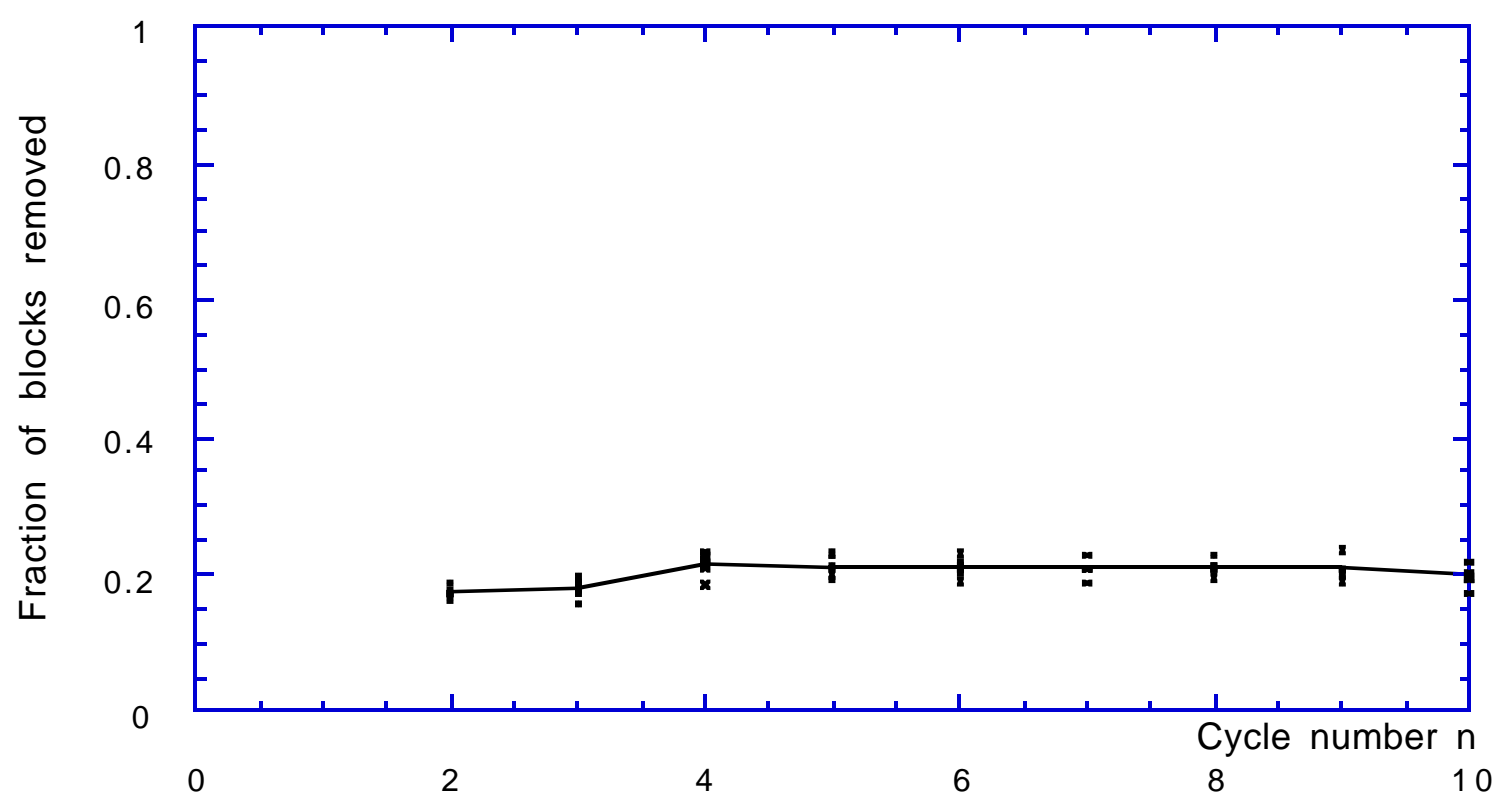

Figure 4.17b: Plot of the number of blocks lifted in cycle $n$ and removed in cycle $n+1$, (as a fraction of the total number of blocks removed in cycle $n+1$ ) Vs. the cycle number $n$, for thickness parameter $\mathrm{T}=1.5$ and maximum pressure $P_{\max }=P_{1}+0.03$ for each cycle. 


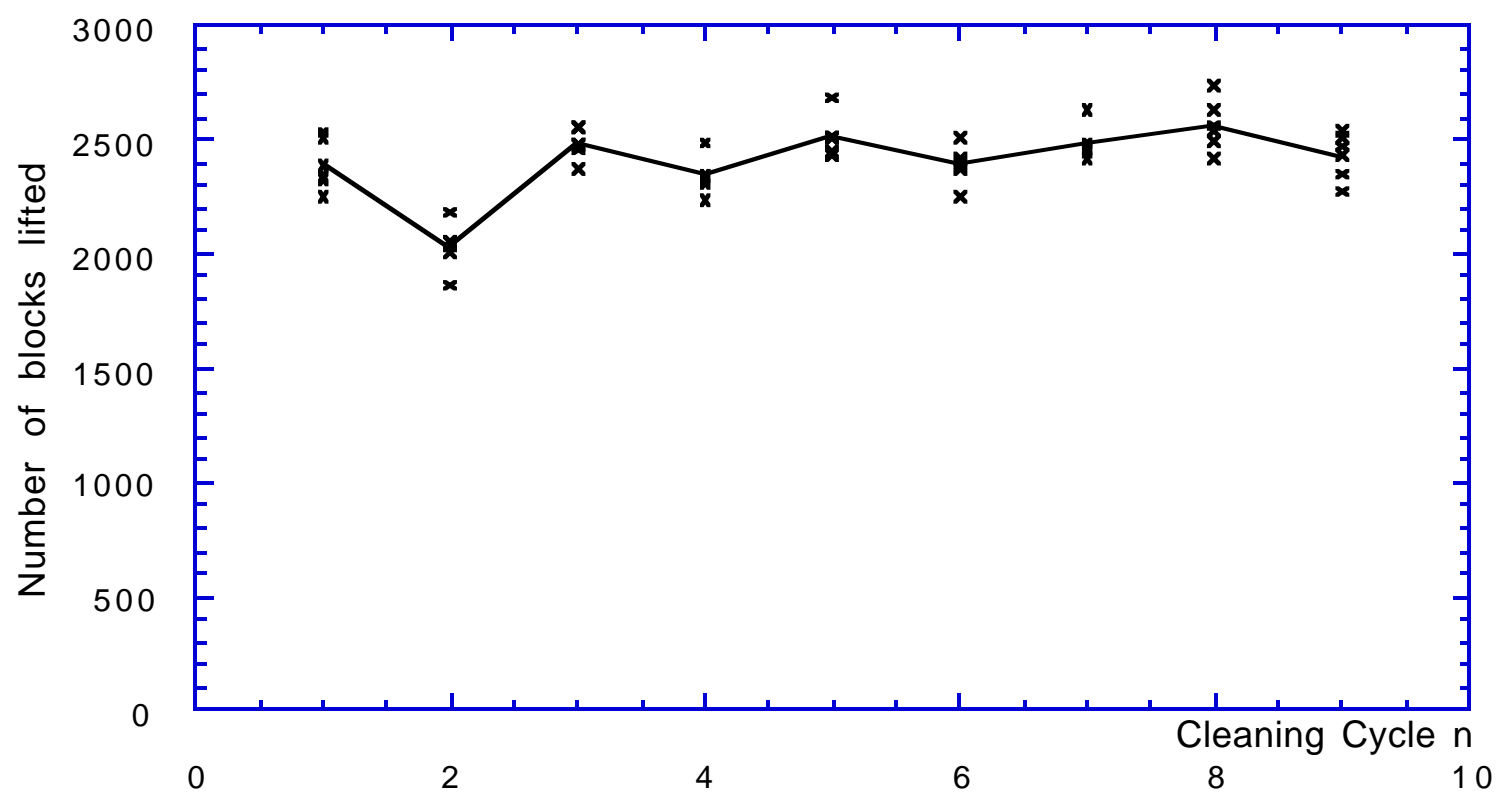

Figure 4.18a: Plot of total number of blocks lifted in cycle n Vs. the cycle number n, for thickness parameter T=1.5 and maximum pressure $P_{\max }=P_{\frac{1}{2}}+0.02$ for each cycle.

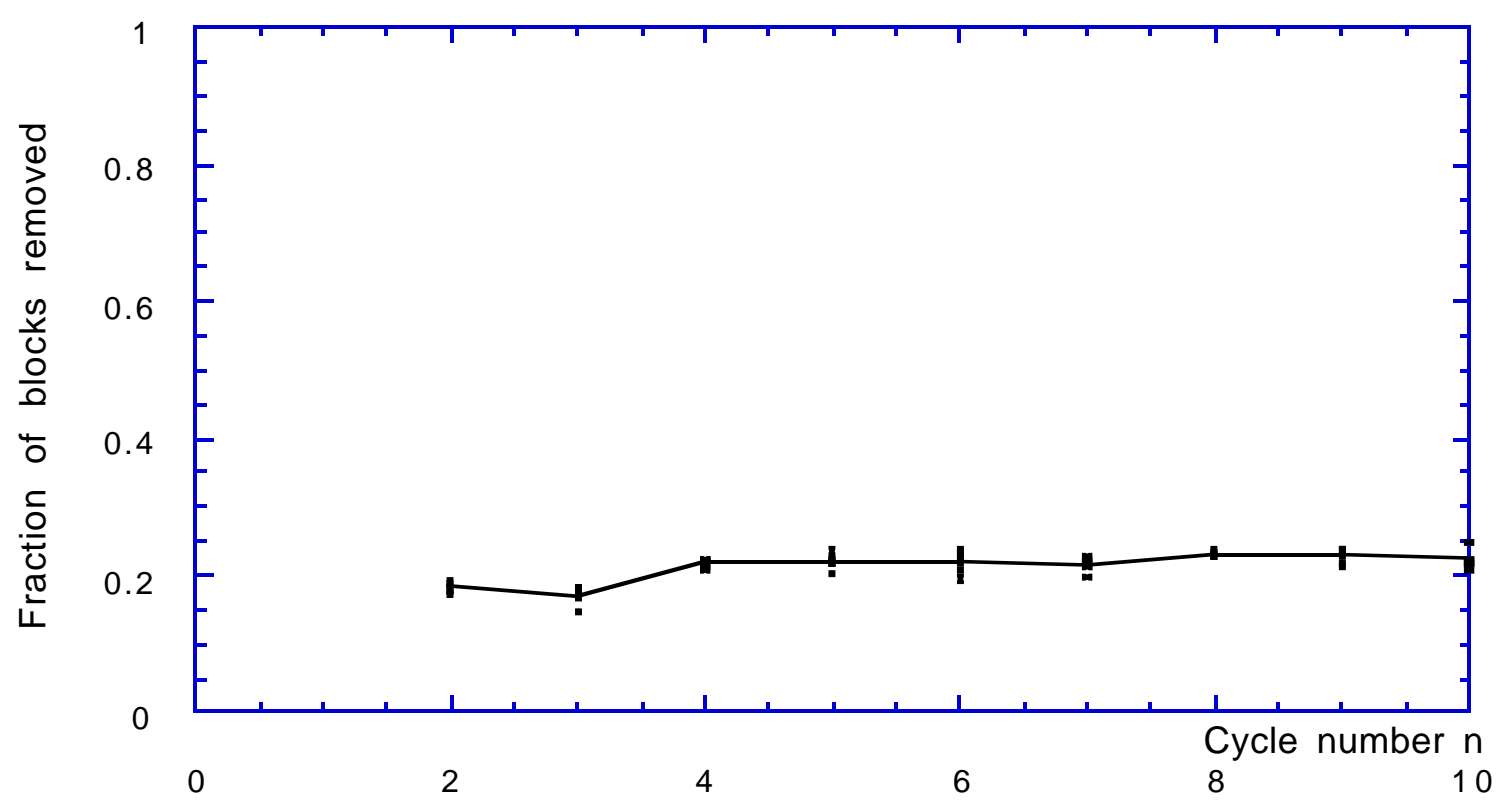

Figure 4.18b: Plot of the number of blocks lifted in cycle $\mathrm{n}$ and removed in cycle $\mathrm{n}+1$, (as a fraction of the total number of blocks removed in cycle $n+1$ ) Vs. the cycle number $n$, for thickness parameter $\mathrm{T}=1.5$ and maximum pressure $P_{\max }=P_{1}+0.02$ for each cycle. 


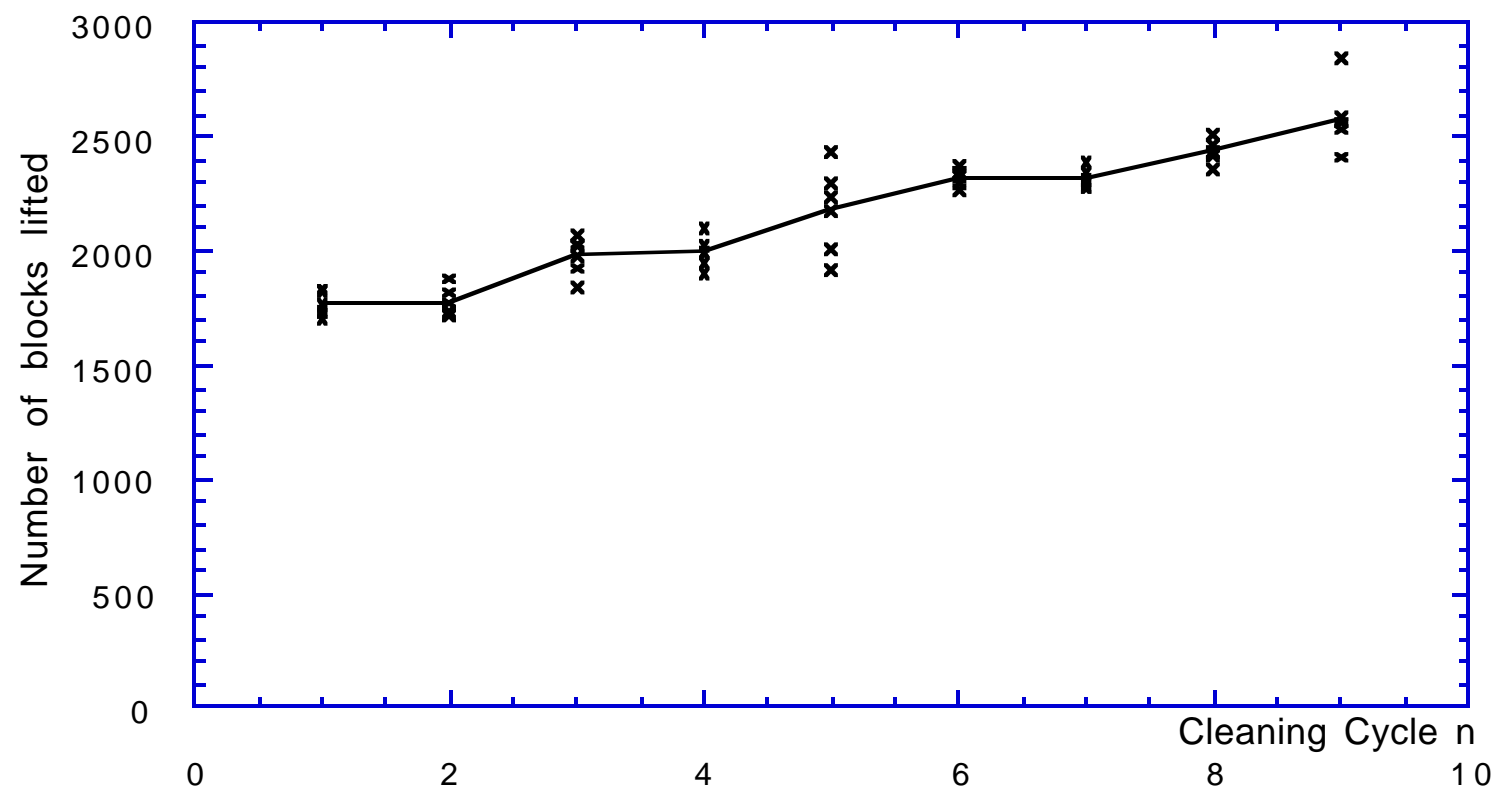

Figure 4.19a: Plot of total number of blocks lifted in cycle $\mathrm{n}$ Vs. the cycle number $\mathrm{n}$, for thickness parameter $\mathrm{T}=1.5$ and maximum pressure $P_{\max }=P_{\frac{1}{2}}+0.0$ for each cycle.

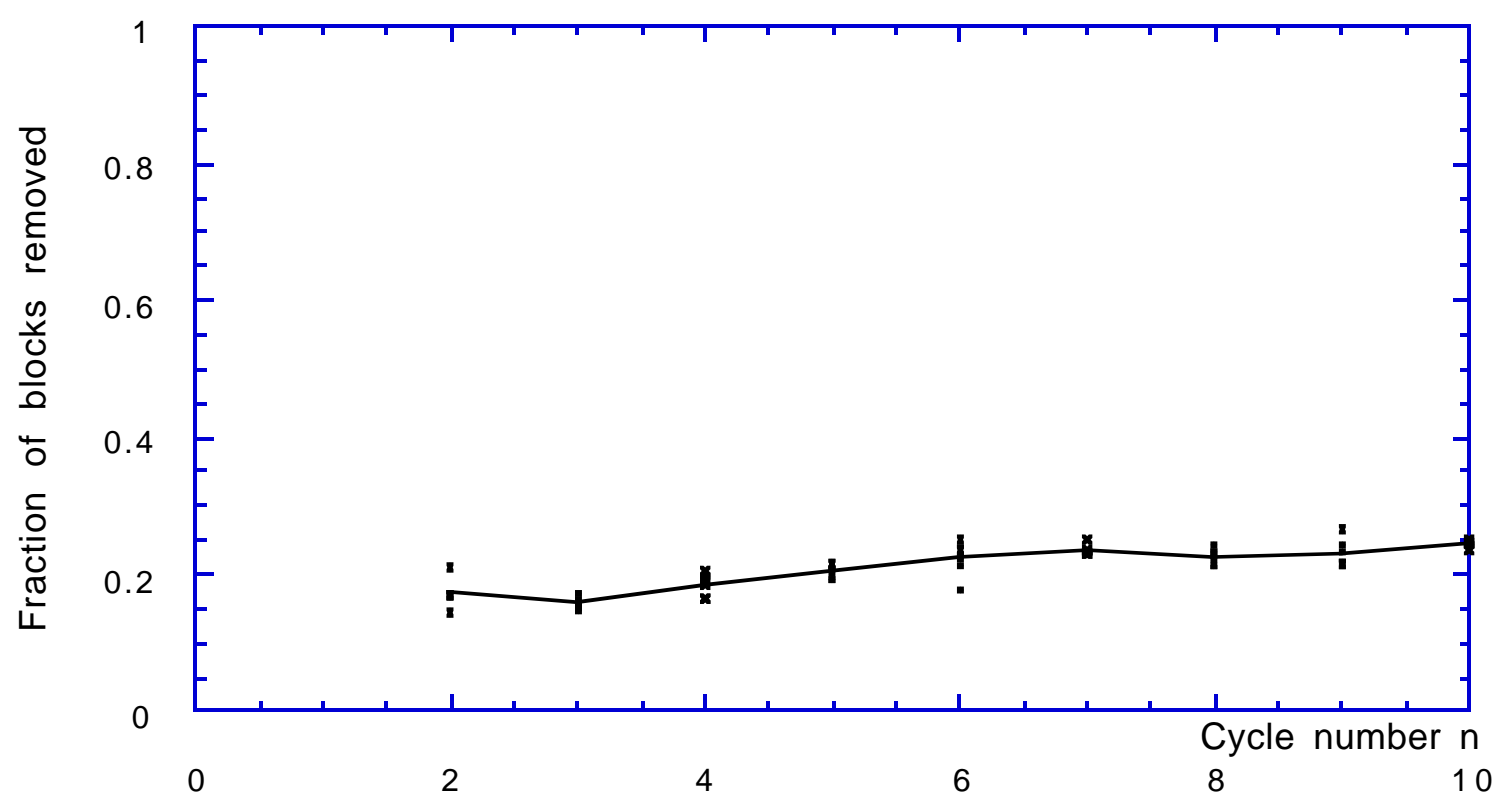

Figure 4.19b: Plot of the number of blocks lifted in cycle $n$ and removed in cycle $n+1$, (as a fraction of the total number of blocks removed in cycle $n+1$ ) Vs. the cycle number $n$, for thickness parameter $\mathrm{T}=1.5$ and maximum pressure $P_{\max }=P_{1}+0.0$ for each cycle. 


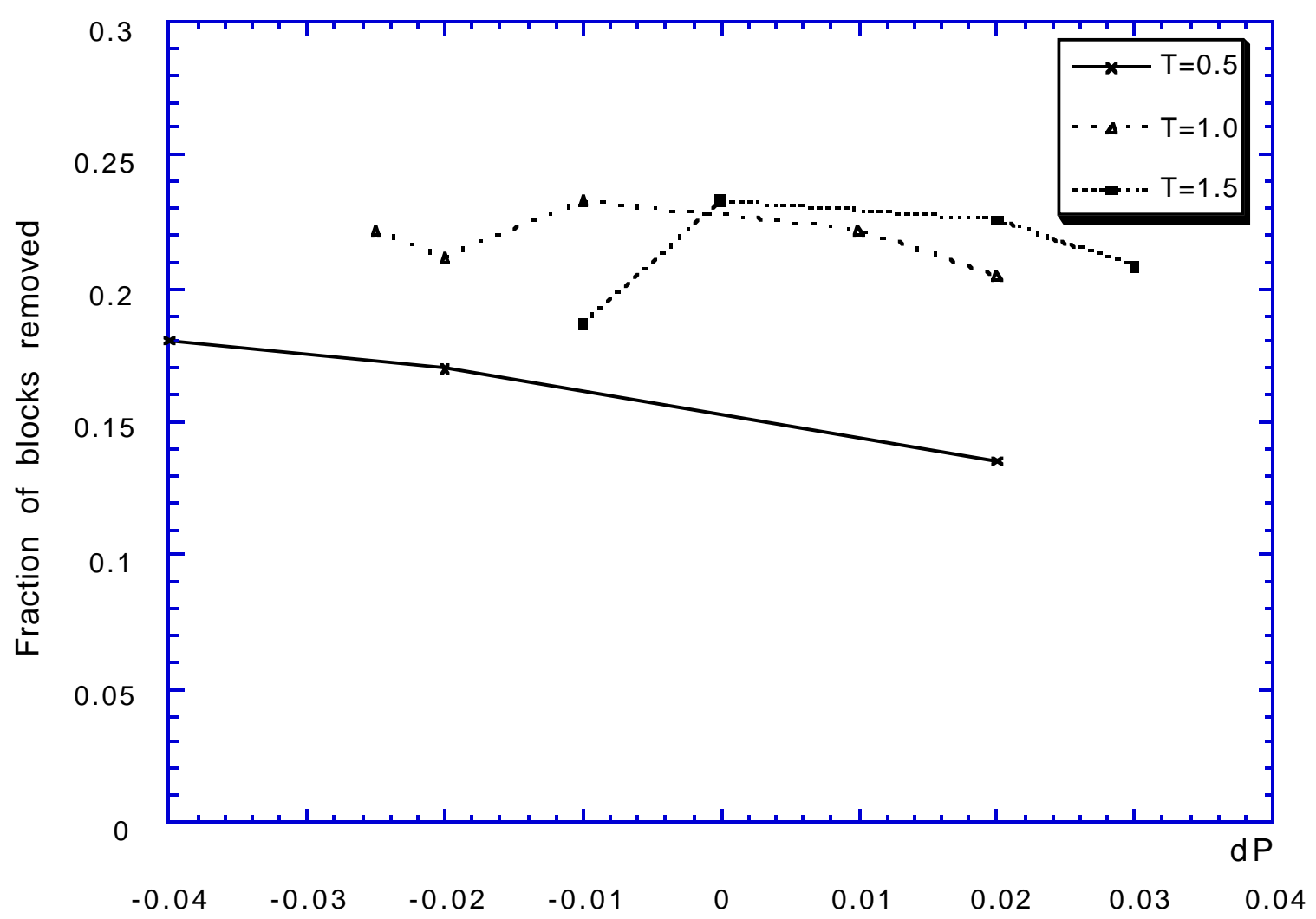

Figure 4.20: A summarized plot of Figures 4.11b-4.19b, showing trends in the fraction of blocks that are lifted in one cycle and removed in the next cycle, (average over the last few cycles) Vs. differential pressure $\mathrm{dP}$, for the three thickness parameters $\mathrm{T}$.

\subsection{Discussion of trends in Figure 4.20}

The results in the Figures 4.11b-4.19b are summarized in Figure 4.20. The figure shows the trends in the fraction of blocks which are lifted in one cycle and removed in the next (averaged over the last few cycles) against the differential pressure dP. The plot shows that for higher differential pressures (i.e. higher maximum applied pressures) as the thickness parameter increases, the fraction of blocks which are lifted in cycle $\mathrm{n}$ and removed in cycle $\mathrm{n}+1$ increases. This indicates that when cohesive forces are large in comparison with the 
adhesive forces, a block may be lifted in one cycle but removed only in the next cycle. This is because, for this block even though its adhesive bond breaks in one cycle, the block may still be attached to neighboring blocks (which are attached to the substrate) with strong cohesive bonds. Hence the block is only lifted in this cycle. During the next cycle the cohesive bonds connecting this block, weakened because they now have to sustain greater stress, are more likely to break, so the block is removed in this cycle.

\subsection{Conclusions from Figures 4.10 and 4.20}

The summarized plots in Figures 4.10 and 4.20, indicate that at higher applied pressures as the thickness parameter $\mathrm{T}$ is increased, out of the total number of blocks that are removed in a given cycle $n+1$, there are fewer of those blocks which have been removed in the previous cycle $n$, and more of those blocks which have been lifted in the previous cycle $n$. Thus for filter cakes which have stronger cohesive forces as compared to adhesive (i.e. larger T), a large fraction of the blocks that are removed in a given cycle, comprise of blocks which have been lifted in the previous cycle. This pattern of a large number of blocks being removed in every other cycle, occurs for larger thickness parameters at higher applied pressures. This result is further confirmed by the analysis undertaken in Chapter Five. 


\section{Chapter Five \\ Cleaning Cycle Analysis II}

\subsection{Introduction}

This chapter furthers the analysis undertaken in Chapter Four by analyzing the trends in the filter cake removal, in context of the number of times (frequency) each block is removed in the 10 cleaning cycles. Each cleaning cycle results in some blocks being removed from the layer. Before starting the next cycle the filter cake layer is regenerated (to the same height) in those areas where blocks have been removed, but left unchanged in those areas where the blocks have not been removed, in the previous cycle. The latter areas, shielded from new deposition, contain blocks with broken adhesive bonds. The damaged adhesive bonds make these blocks weaker, and hence more likely to be removed in the next cycle.

\subsection{Cleaning Patterns}

The fine scale model grids the filter cake layer into rectangular blocks and assigns to each block a position coordinate $(i, j)$. A computer program is written to count the number of cycles in which a particular block is removed. The position of each block on the layer is plotted in Figures 5.1, 5.2 and 5.3, along with the number of cycles in which it has been removed. This is done by choosing a gray scale in which dark black regions represent areas where blocks have been removed in all 10 cycles, and the lightest gray regions represent areas in which blocks have been removed in only 1 cycle. The white regions then correspond to blocks which have not been cleaned in any cycle. The patterns thus generated were analyzed, for a variety of applied pressures for each of the three thickness parameters. The orientation of the patterns is such that periodic boundary conditions connect the side edges, and the strongly adhesive bonds are just off the figure on the top and the bottom. 


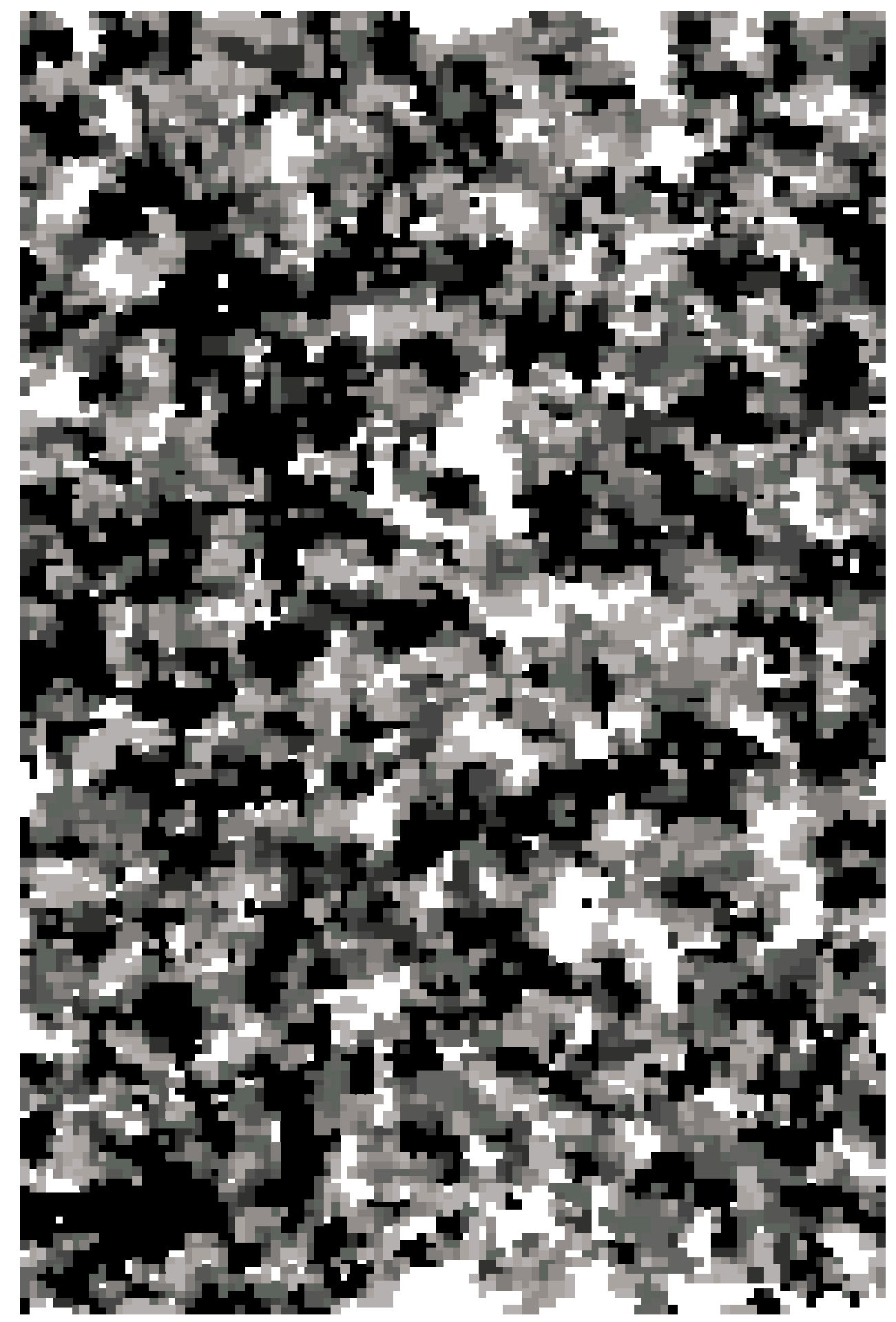

Figure 5.1a: Plot of the filter cake layer for thickness parameter $\mathrm{T}=0.5$ and maximum pressure $P_{\max }=P_{1}+0.02$. The blackest regions represent blocks which are removed all 10 times while the lightest gray regions represent blocks which are removed in only 1 cycle. 


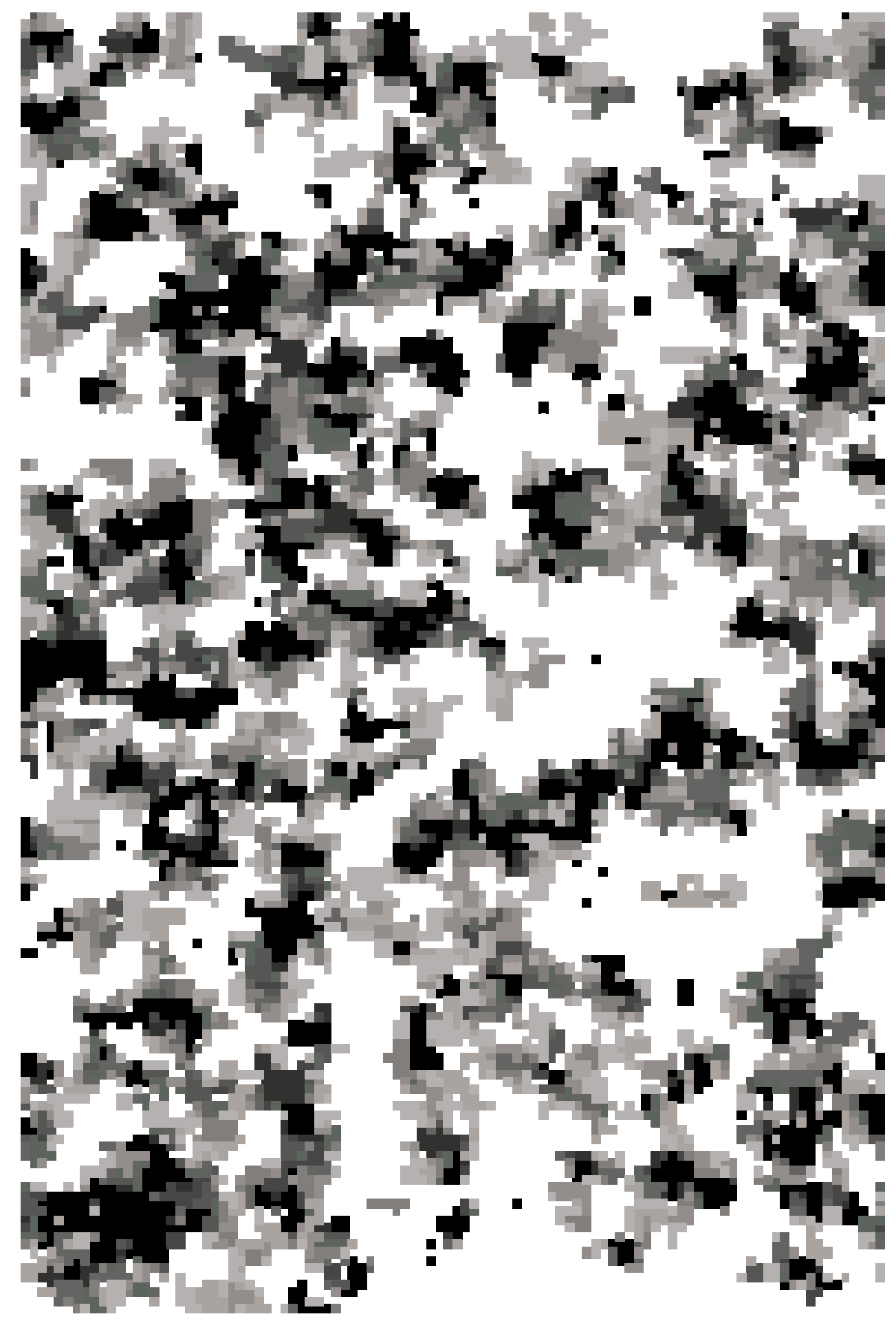

Figure 5.1b: Plot of the filter cake layer for thickness parameter $\mathrm{T}=0.5$ and maximum pressure $P_{\max }=P_{1}-0.02$. The blackest regions represent blocks which are removed all 10 times while the lightest gray regions represent blocks which are removed in only 1 cycle. 


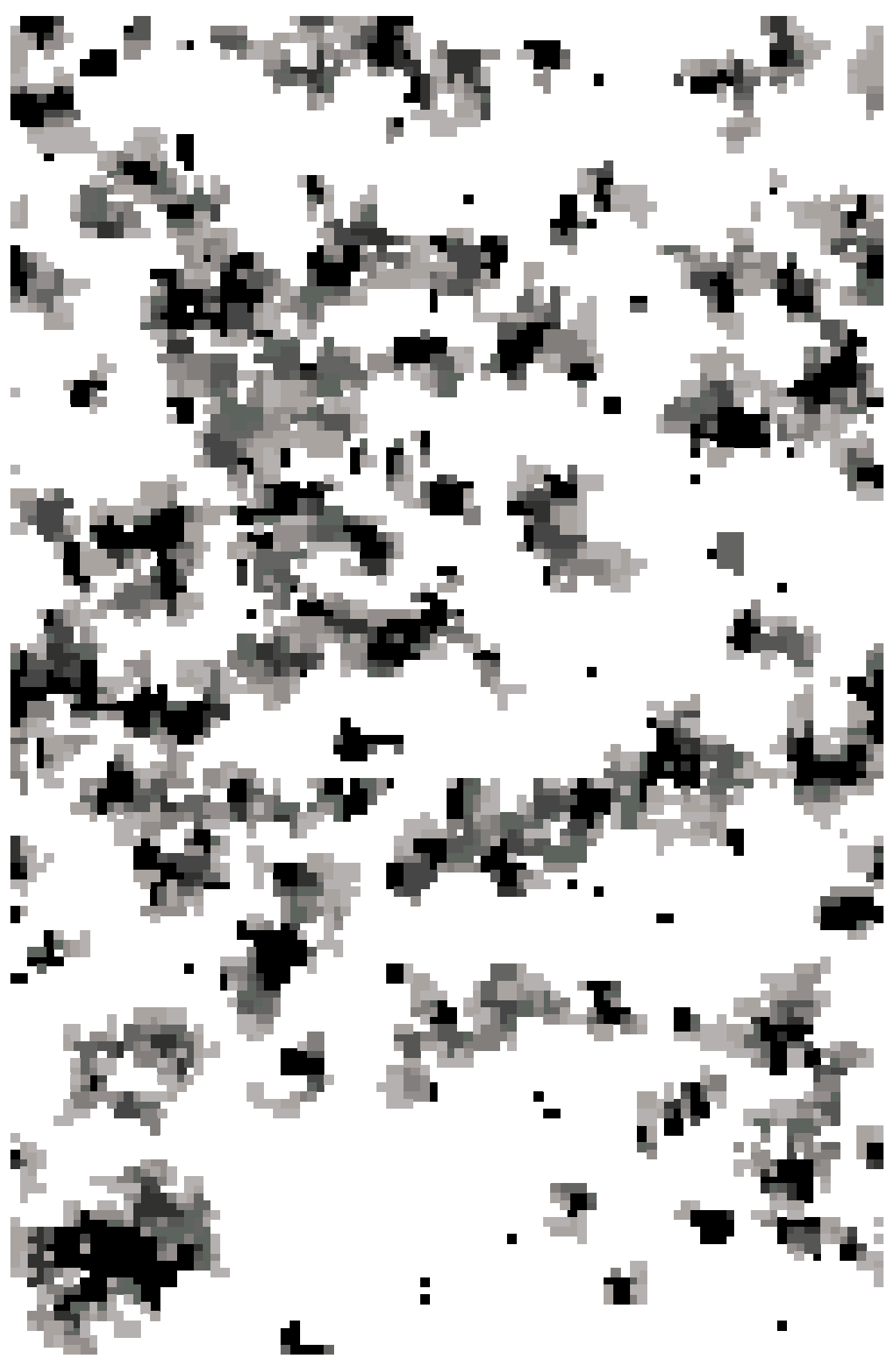

Figure 5.1c: Plot of the filter cake layer for thickness parameter $\mathrm{T}=0.5$ and maximum pressure $P_{\max }=P_{1}-0.04$. The blackest regions represent blocks which are removed all 10 times while the lightest gray regions represent blocks which are removed in only 1 cycle. 


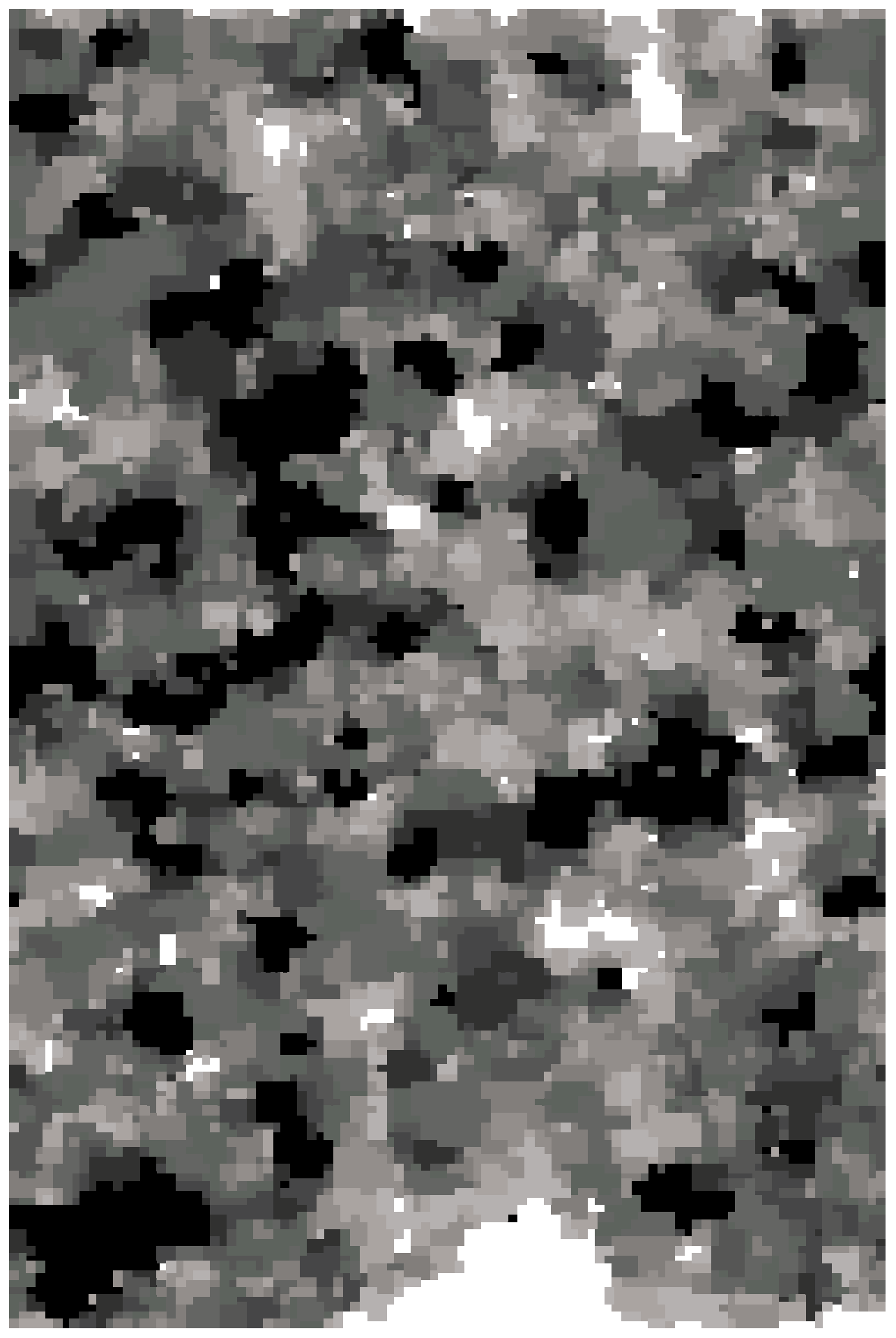

Figure 5.2a: Plot of the filter cake layer for thickness parameter $\mathrm{T}=1.0$ and maximum pressure $P_{\max }=P_{1}+0.02$. The blackest regions represent blocks which are removed all 10 times while the lightest gray regions represent blocks which are removed in only 1 cycle. 


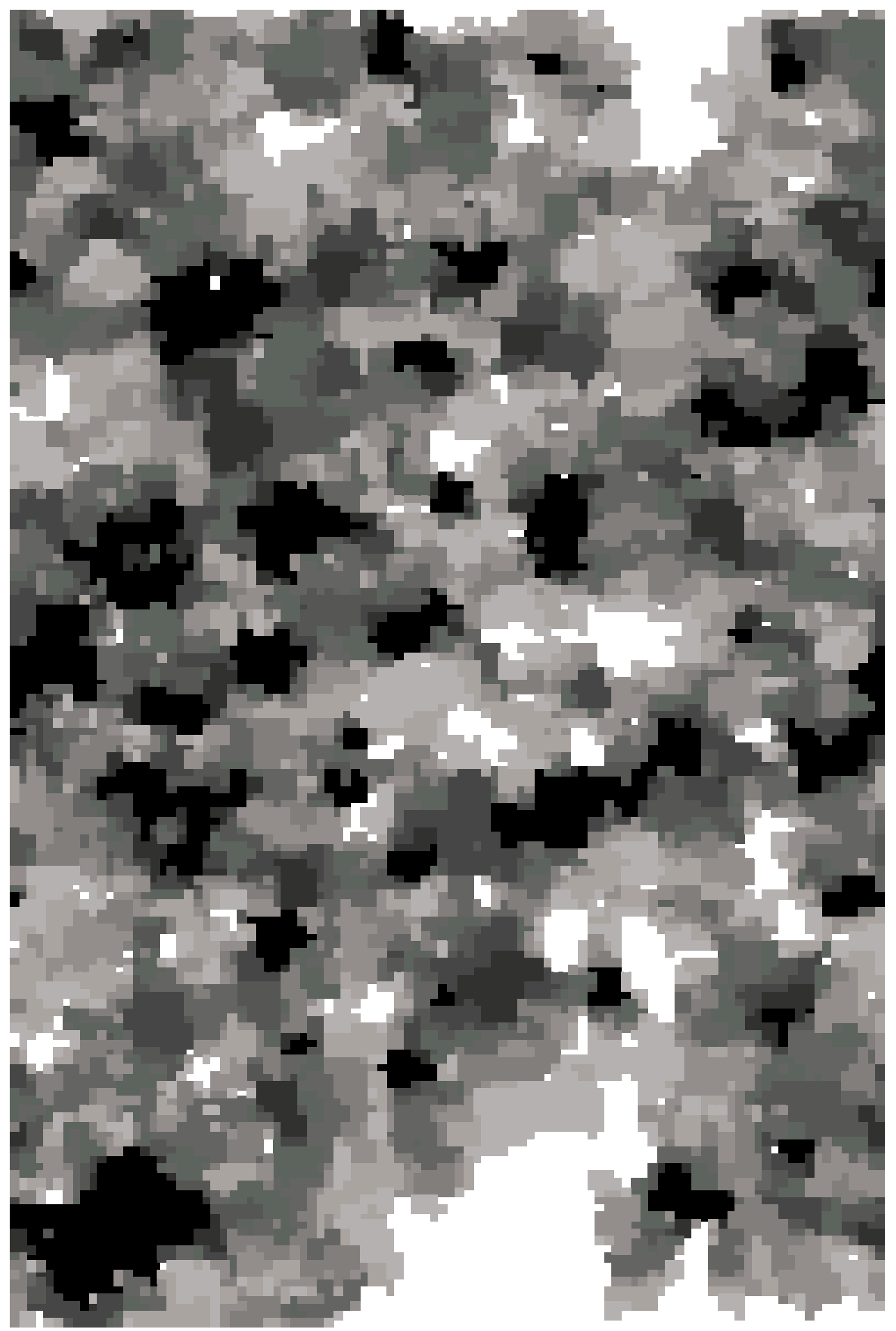

Figure 5.2b: Plot of the filter cake layer for thickness parameter $\mathrm{T}=1.0$ and maximum pressure $P_{\max }=P_{1}+0.01$. The blackest regions represent blocks which are removed all 10 times while the lightest gray regions represent blocks which are removed in only 1 cycle. 


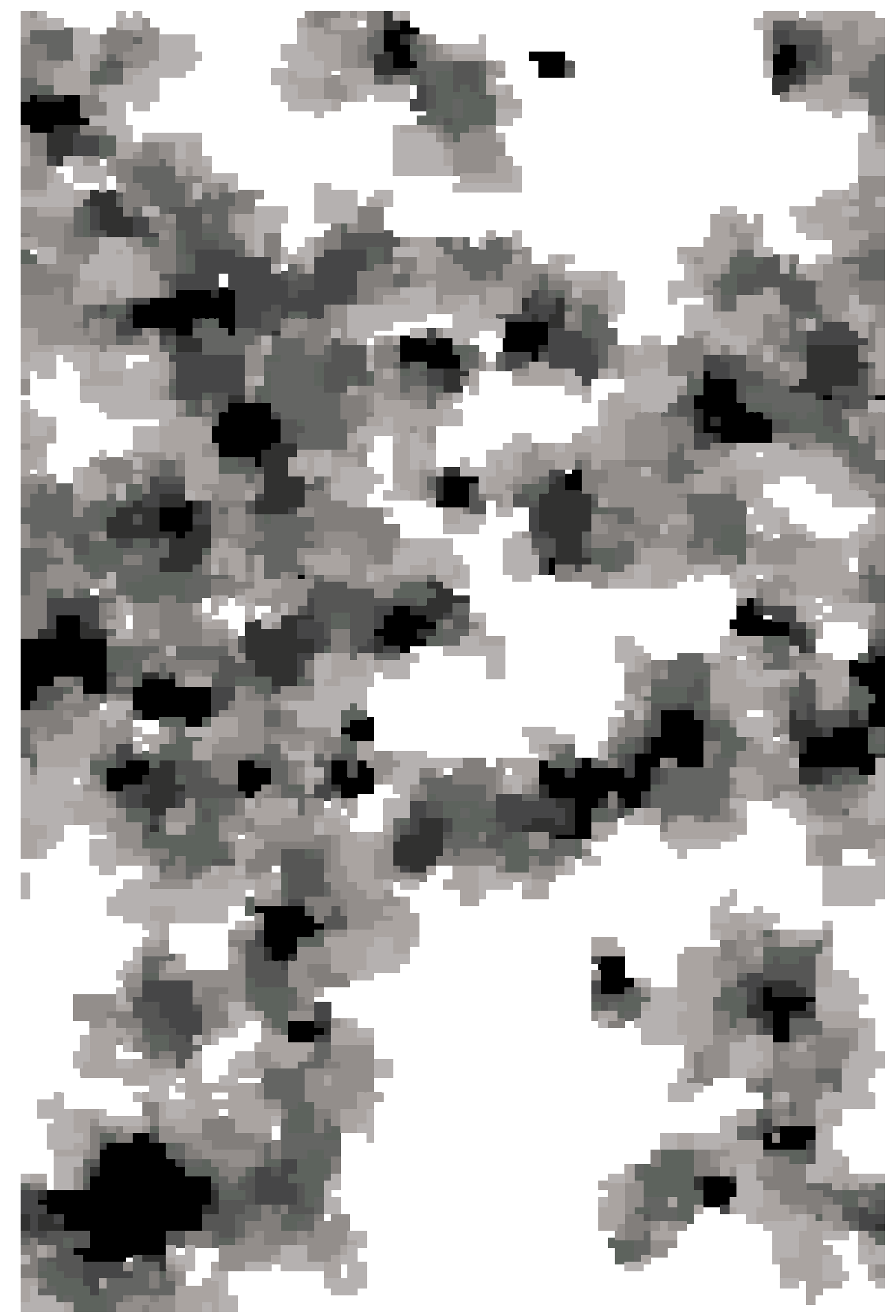

Figure 5.2c: Plot of the filter cake layer for thickness parameter $\mathrm{T}=1.0$ and maximum pressure $P_{\max }=P_{\frac{1}{2}}-0.01$. The blackest regions represent blocks which are removed all 10 times while the lightest gray regions represent blocks which are removed in only 1 cycle. 


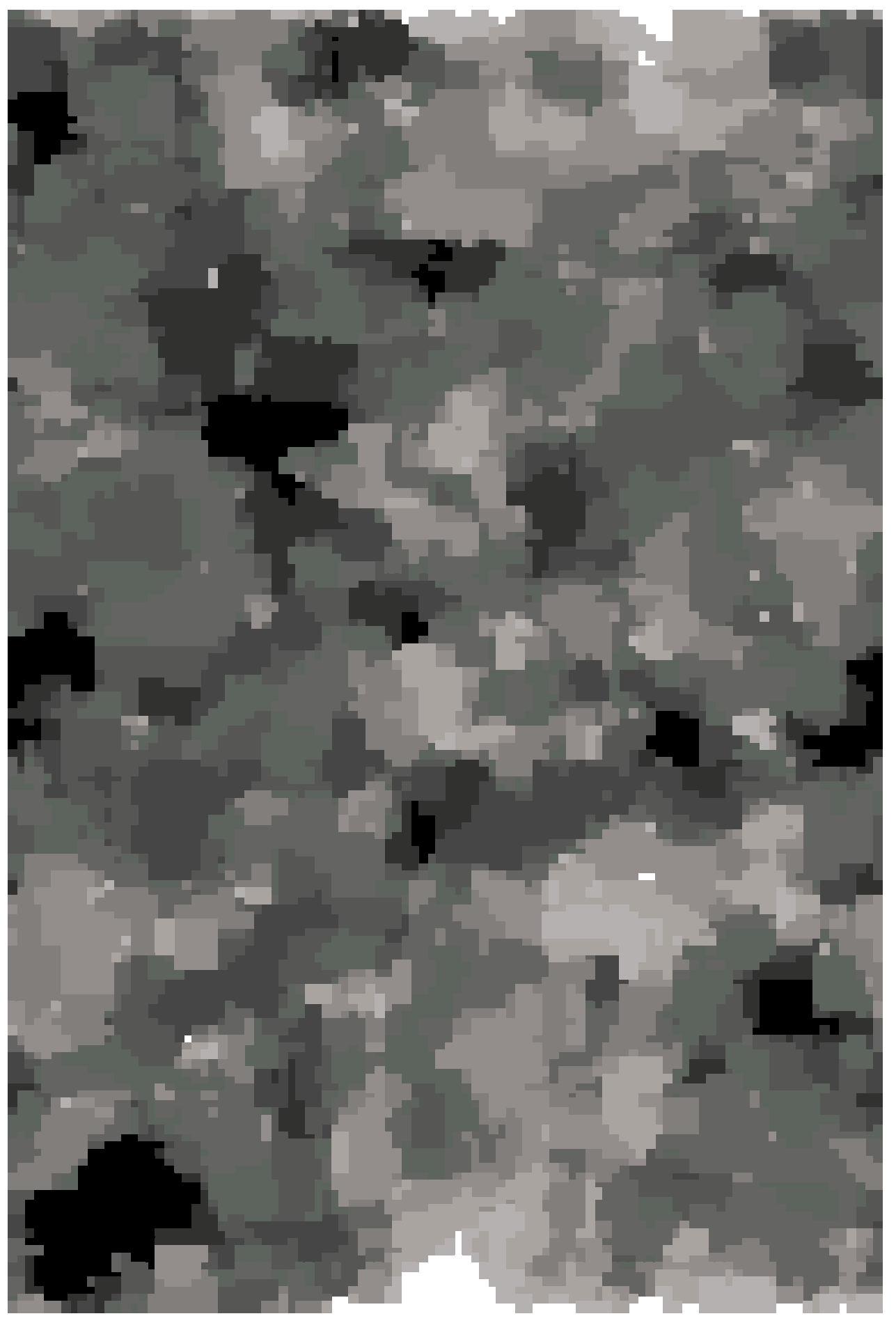

Figure 5.3a: Plot of the filter cake layer for thickness parameter $\mathrm{T}=1.5$ and maximum pressure $P_{\max }=P_{1}+0.03$. The blackest regions represent blocks which are removed all 10 times while the lightest gray regions represent blocks which are removed in only 1 cycle. 


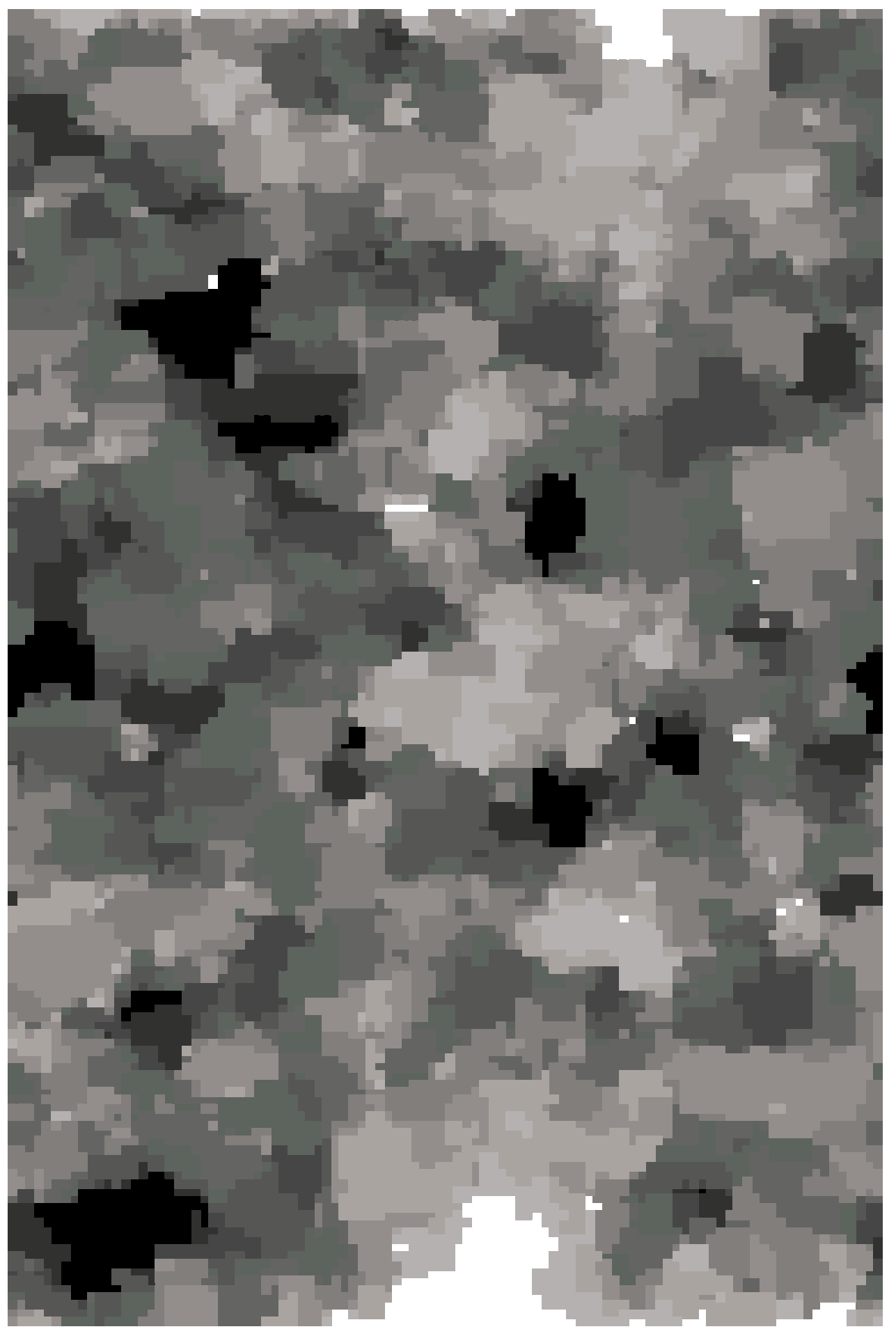

Figure 5.3b: Plot of the filter cake layer for thickness parameter $\mathrm{T}=1.5$ and maximum pressure $P_{\max }=P_{1}+0.02$. The blackest regions represent blocks which are removed all 10 times while the lightest gray regions represent blocks which are removed in only 1 cycle. 


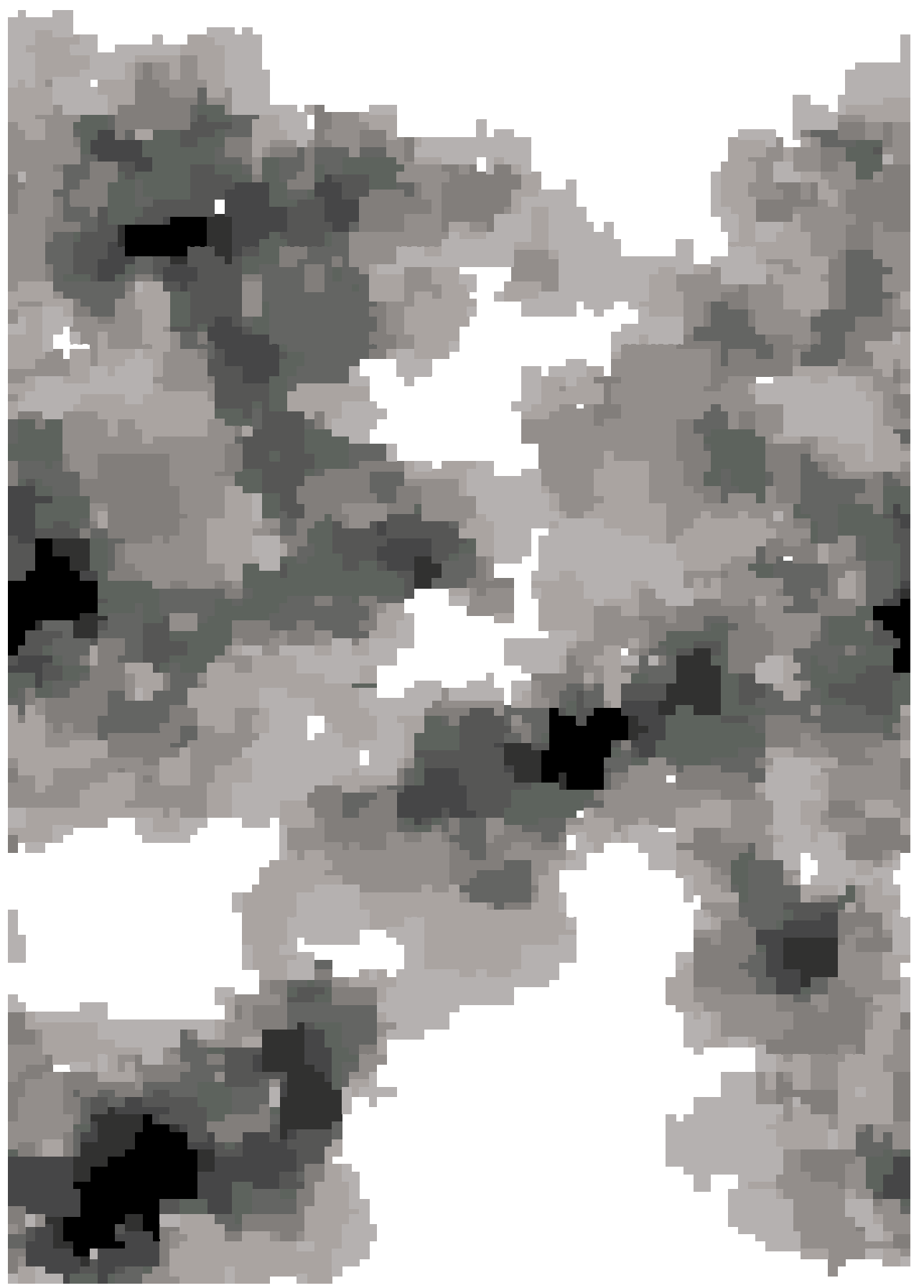

Figure 5.3c: Plot of the filter cake layer for thickness parameter $\mathrm{T}=1.5$ and maximum pressure $P_{\max }=P_{1}+0.0$. The blackest regions represent blocks which are removed all 10 times while the lightest gray regions represent blocks which are removed in only 1 cycle. 


\subsection{Discussion of Plots}

The above patterns in Figures 5.1(a-c), 5.2(a-c) and 5.3(a-c) show shaded regions which reflect the corresponding frequencies (number of times) with which the blocks in those areas have been cleaned. A comparison of the plots for a given thickness parameter suggests that there is little variation in the size of the shaded regions (patches) with changes in applied pressure. However when the trends among the 3 thickness parameter plots are compared it is observed that the size of the individual shaded regions increases with increasing values of the thickness parameter T. This is consistent with previously observed increase of patch size with increasing thickness [3]. The role of increased cooperative forces is clear. The stronger cohesive forces try to maintain the integrity of the layer, leading to larger sections of the layer being removed at a given pressure.

\subsection{Average Plots}

Figures 5.1-5.3 were graphed using data from the first realizations only. Figures 5.4-5.5 now examine the average behavior of the 5 realizations. For each realization the total number of blocks that are removed with a particular frequency are noted. This essentially involves counting all the sites which are shaded a particular color in one of the Figures 5.15.3. The total number of sites removed are plotted against the frequency (number of times) with which they were removed for all 5 realizations in Figures 5.1(a-c), 5.2(a-c) and 5.3(ac). The averages of the 5 realizations are connected by a straight line, and the resulting trend analyzed. 


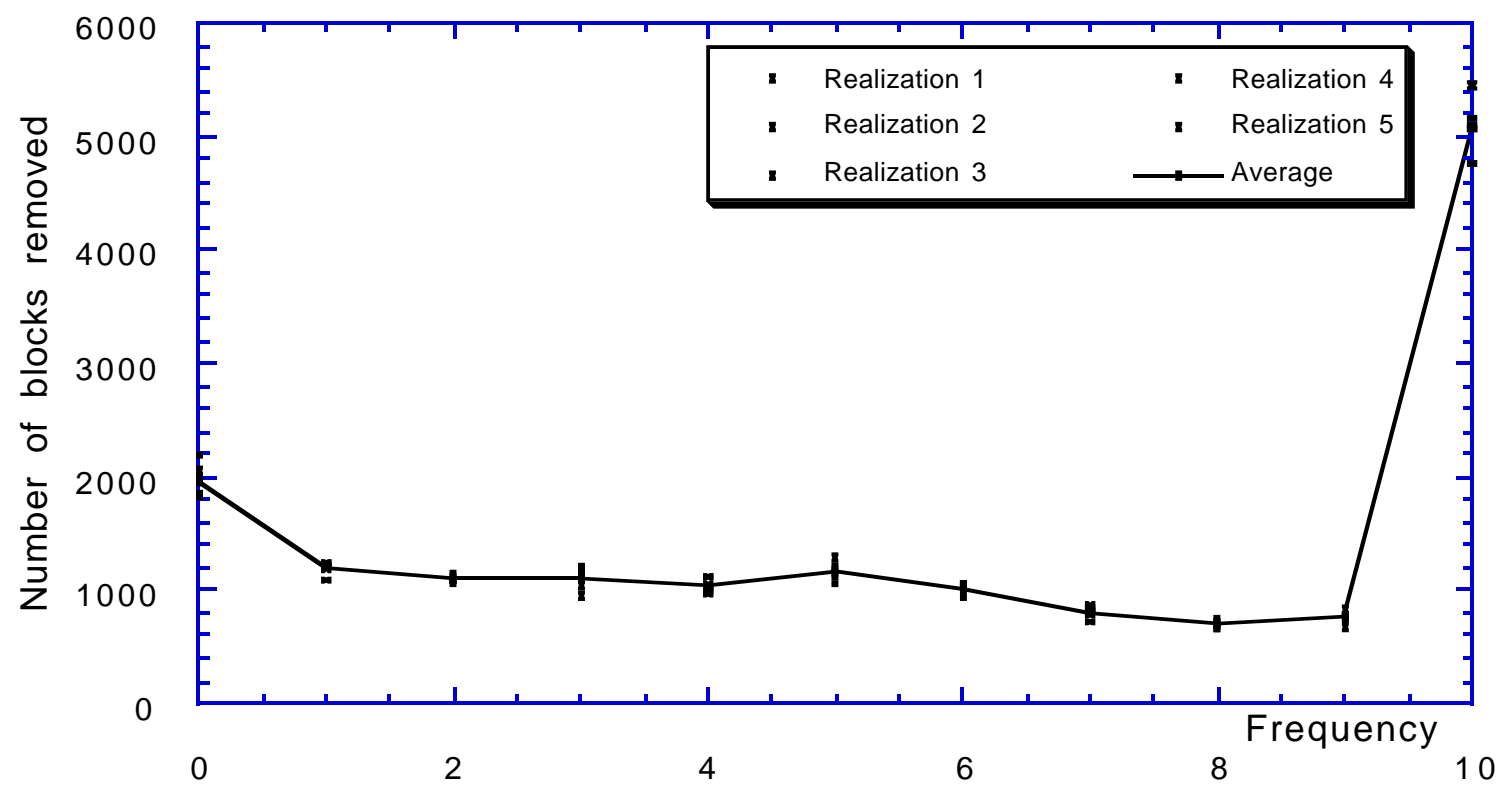

Figure 5.4a: Plot of total number of blocks removed Vs. the frequency (number of cycles) with which they were removed, for $\mathrm{T}=0.5$ and maximum pressure $P_{\max }=P_{\frac{1}{2}}+0.02$.

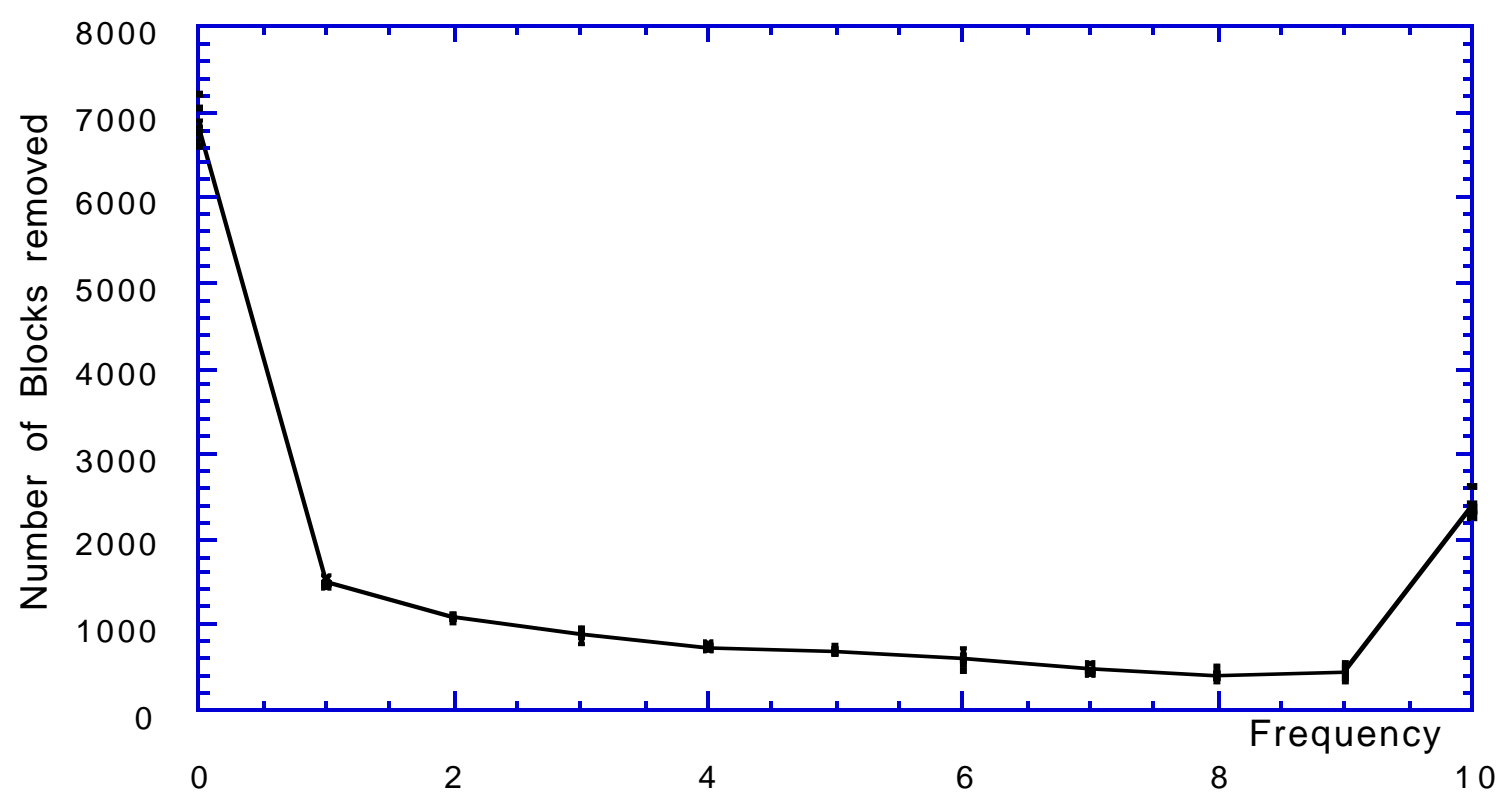

Figure 5.4b: Plot of total number of blocks removed Vs. the frequency (number of cycles) with which they were removed, for $\mathrm{T}=0.5$ and maximum pressure $P_{\max }=P_{\frac{1}{2}}-0.02$. 


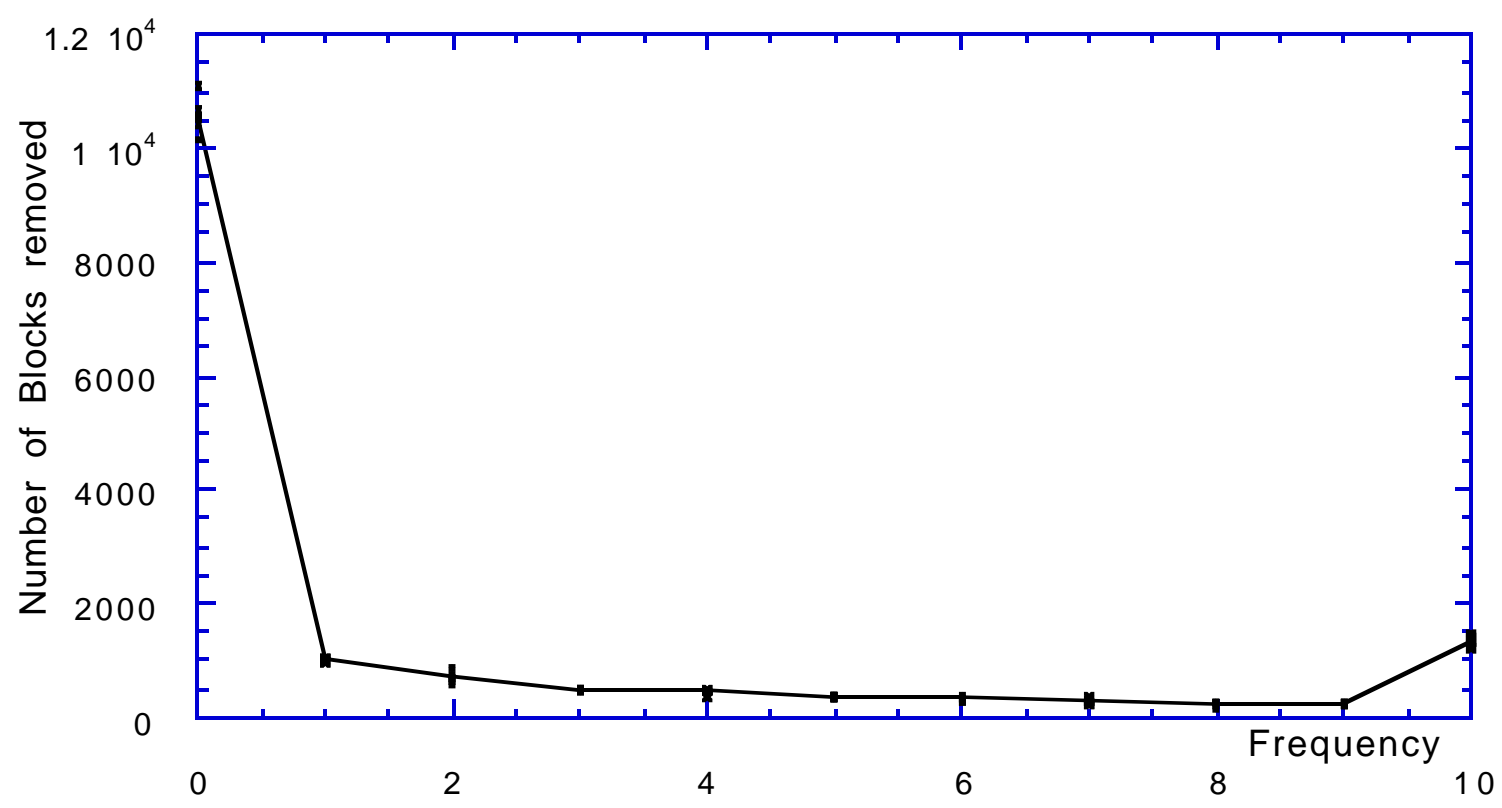

Figure 5.4c: Plot of total number of blocks removed Vs. the frequency (number of cycles) with which they were removed, for $\mathrm{T}=0.5$ and maximum pressure $P_{\max }=P_{\frac{1}{2}}-0.04$.

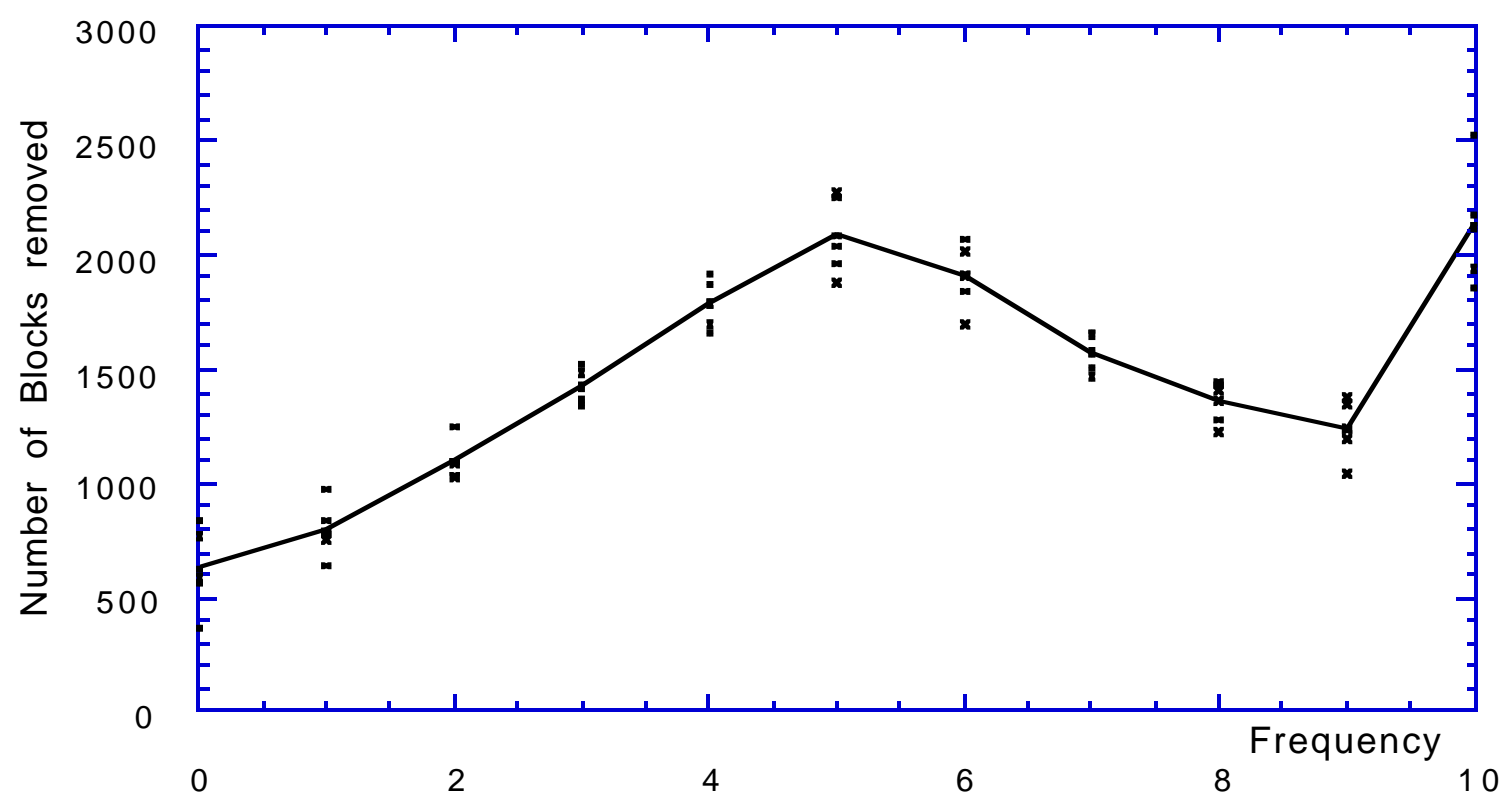

Figure 5.5a: Plot of total number of blocks removed Vs. the frequency (number of cycles) with which they were removed, for $\mathrm{T}=1.0$ and maximum pressure $P_{\max }=P_{\frac{1}{2}}+0.02$. 


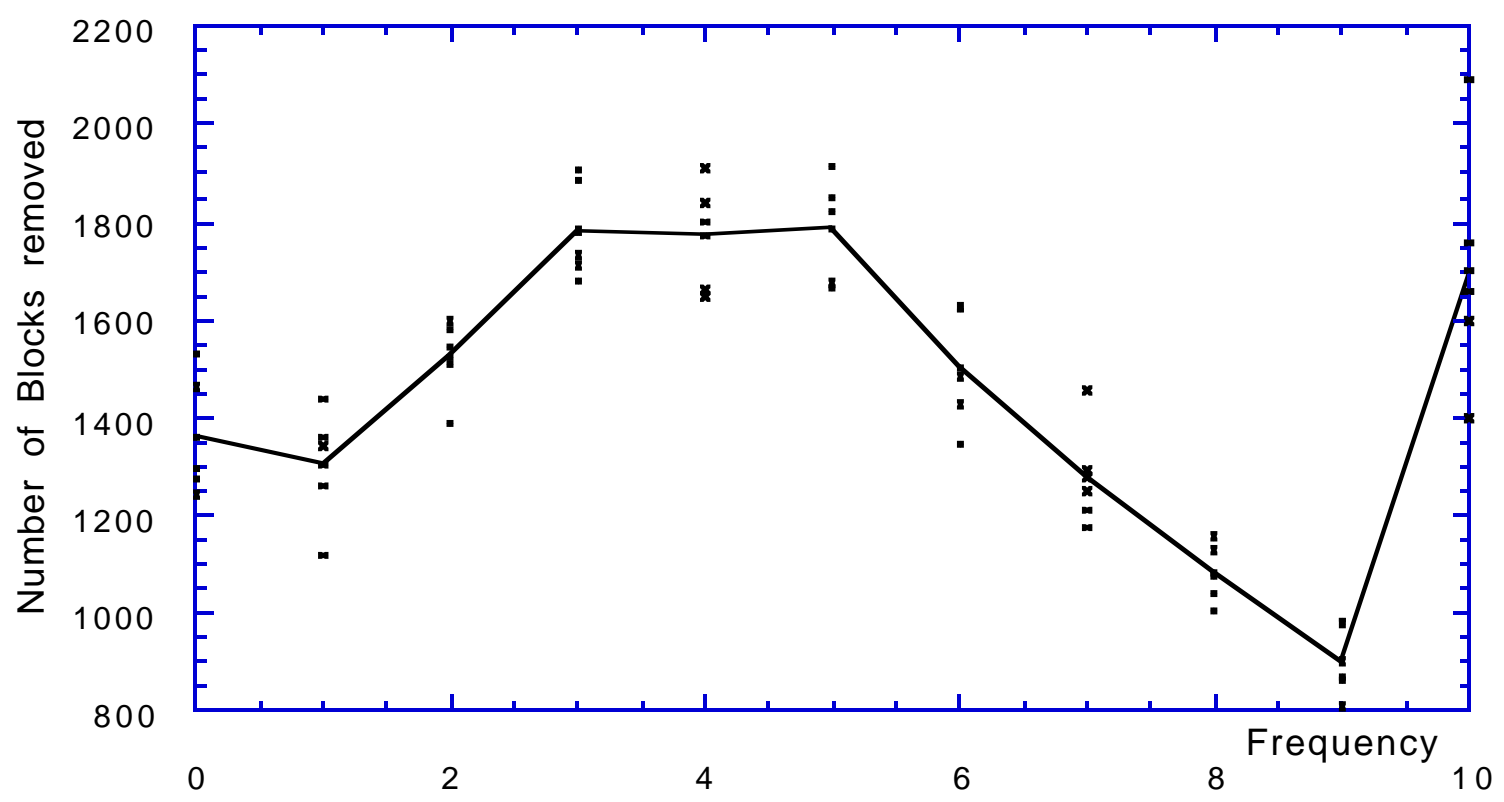

Figure 5.5b: Plot of total number of blocks removed Vs. the frequency (number of cycles) with which they were removed, for $\mathrm{T}=1.0$ and maximum pressure $P_{\max }=P_{\frac{1}{2}}+0.01$.

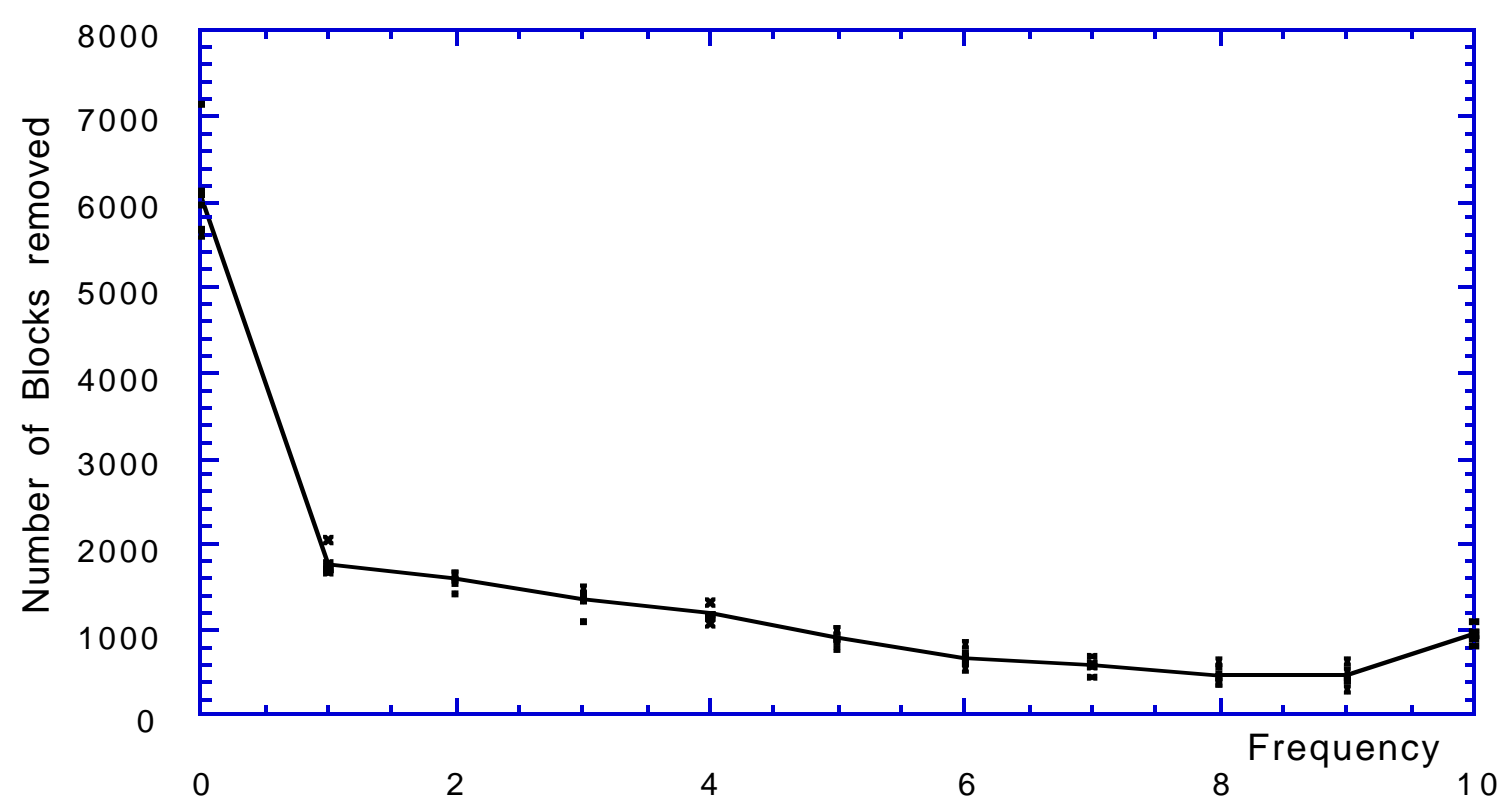

Figure 5.5c: Plot of total number of blocks removed Vs. the frequency (number of cycles) with which they were removed, for $\mathrm{T}=1.0$ and maximum pressure $P_{\max }=P_{\frac{1}{2}}-0.01$. 


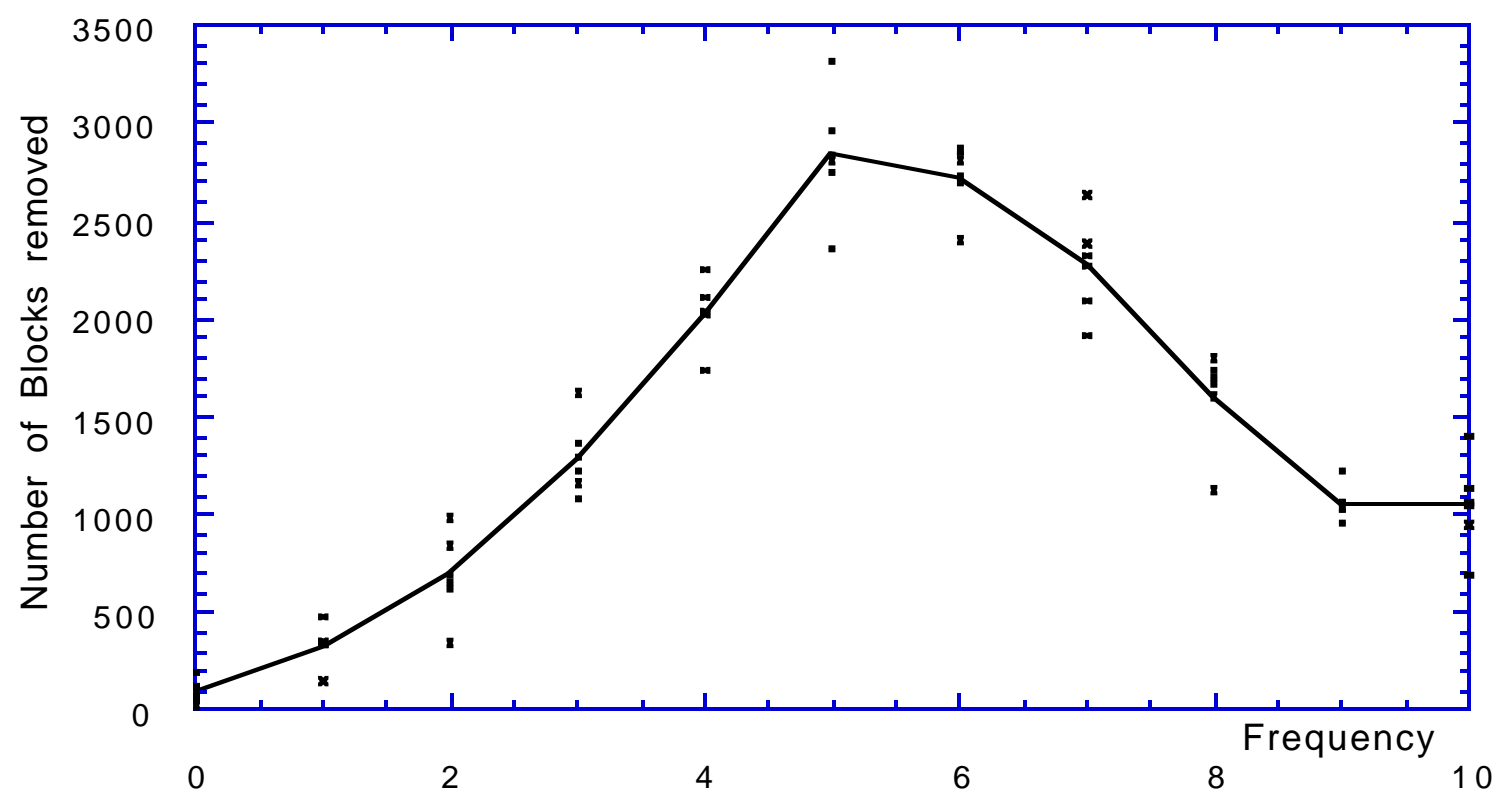

Figure 5.6a: Plot of total number of blocks removed Vs. the frequency (number of cycles) with which they were removed, for $\mathrm{T}=1.5$ and maximum pressure $P_{\max }=P_{\frac{1}{2}}+0.03$.

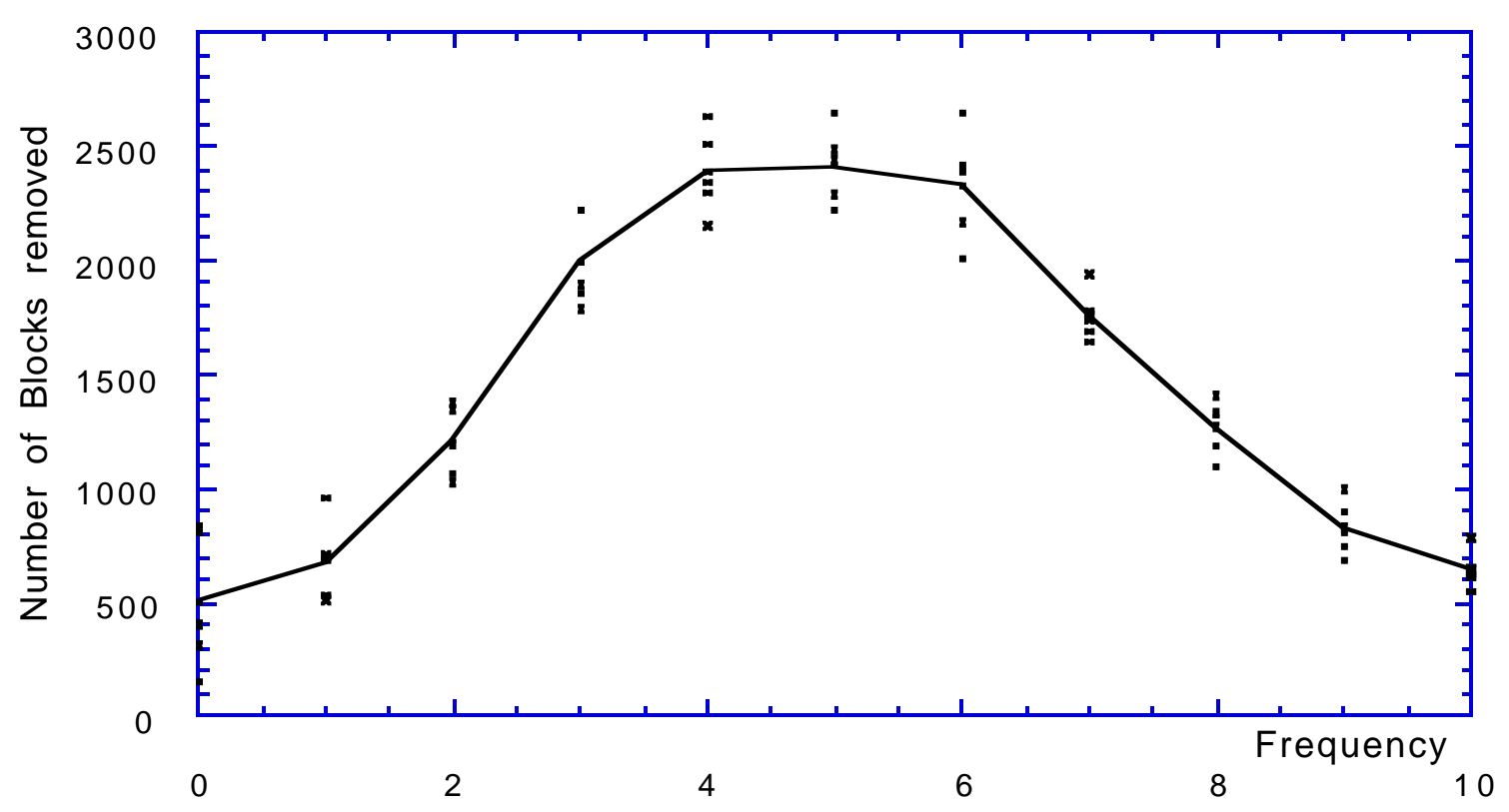

Figure 5.6b: Plot of total number of blocks removed Vs. the frequency (number of cycles) with which they were removed, for $\mathrm{T}=1.5$ and maximum pressure $P_{\max }=P_{\frac{1}{2}}+0.02$. 


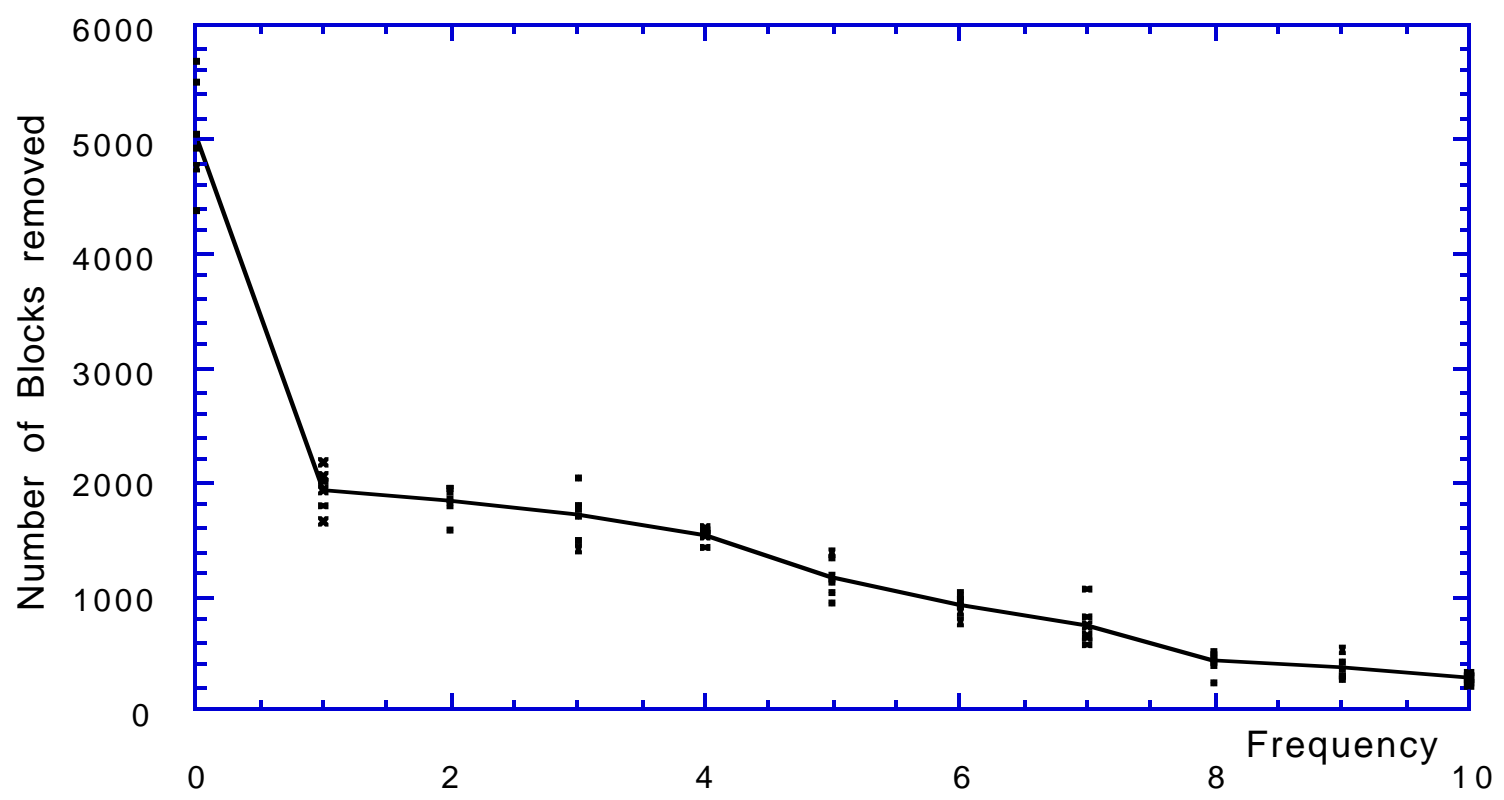

Figure 5.6c: Plot of total number of blocks removed Vs. the frequency (number of cycles) with which they were removed, for $\mathrm{T}=1.5$ and maximum pressure $P_{\max }=P_{\frac{1}{2}}+0.0$.

\subsection{Analysis of the Average plots}

The plots of Figures 5.4 (a-c), 5.5 (a-c) and 5.6 (a-c) show that for a given value of thickness parameter $\mathrm{T}$, the number of blocks that are removed in all 10 cycles increases with increasing values of applied pressure (i.e. increasing values of $\mathrm{dP}$ ). This is as expected since at larger values of applied pressure, more blocks are removed in each cycle. On comparing the trends among the 3 thickness parameters, a hump centered about frequency $=5$, appears for higher thickness parameters $(T=1.0,1.5)$ at higher applied pressures $(\mathrm{dP}=0.02,0.03)$. This suggests that for larger T's at higher pressures some blocks are being removed in 5 cycles out of 10. This implies that these blocks are being removed in every other cycle, i.e. they are lifted in one cycle and then removed in the next. This effect is due to the presence of strong cohesive bonds. For these blocks the adhesive 
bond breaks, but the block remains connected to one or more neighboring blocks by strong cohesive bonds. Thus this block is lifted in one cycle and removed in the next.

\subsection{Cumulative Plots}

The Figures 5.7 (a-c), 5.8 (a-c) and 5.9 (a-c) show the cumulative plots of the number of blocks removed. The plots use that same data as in Section (5.4), but involve only the averages of all 5 realizations. The graphs are cumulative since they plot the number of blocks removed $\mathrm{n}$ or fewer times. The percentage of area removed $\mathrm{n}$ or fewer times is calculated by dividing the number of blocks removed $\mathrm{n}$ or fewer times, by the total number of blocks in the system $(16,000)$. This area is then plotted against the frequency to obtain the graphs in Figures 5.7, 5.8 and 5.9.

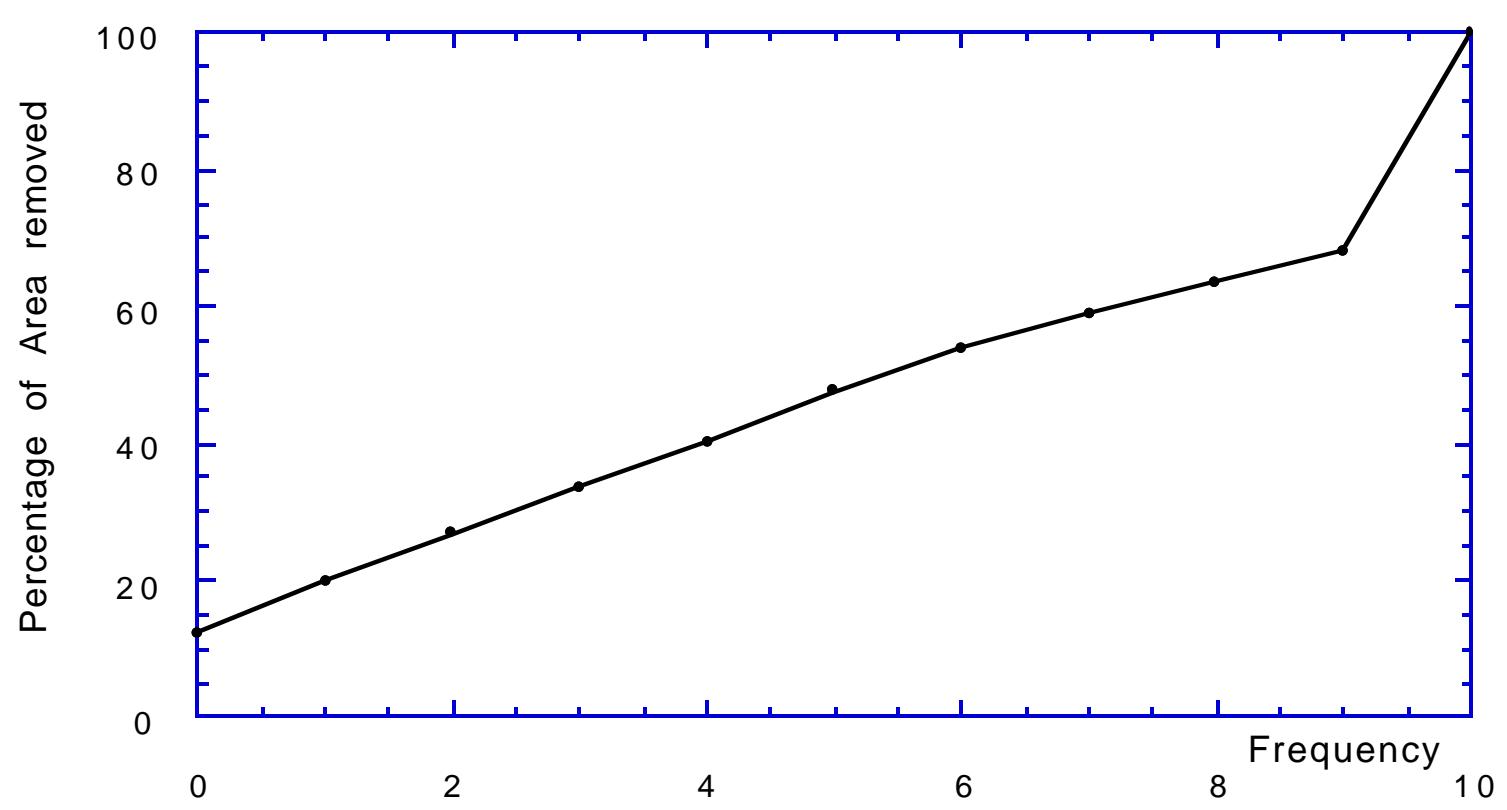

Figure 5.7a: Plot of the percentage of Cumulative area removed Vs. the frequency (number of cycles) of removal, for $\mathrm{T}=0.5$ and maximum pressure $P_{\max }=P_{\frac{1}{2}}+0.02$. 


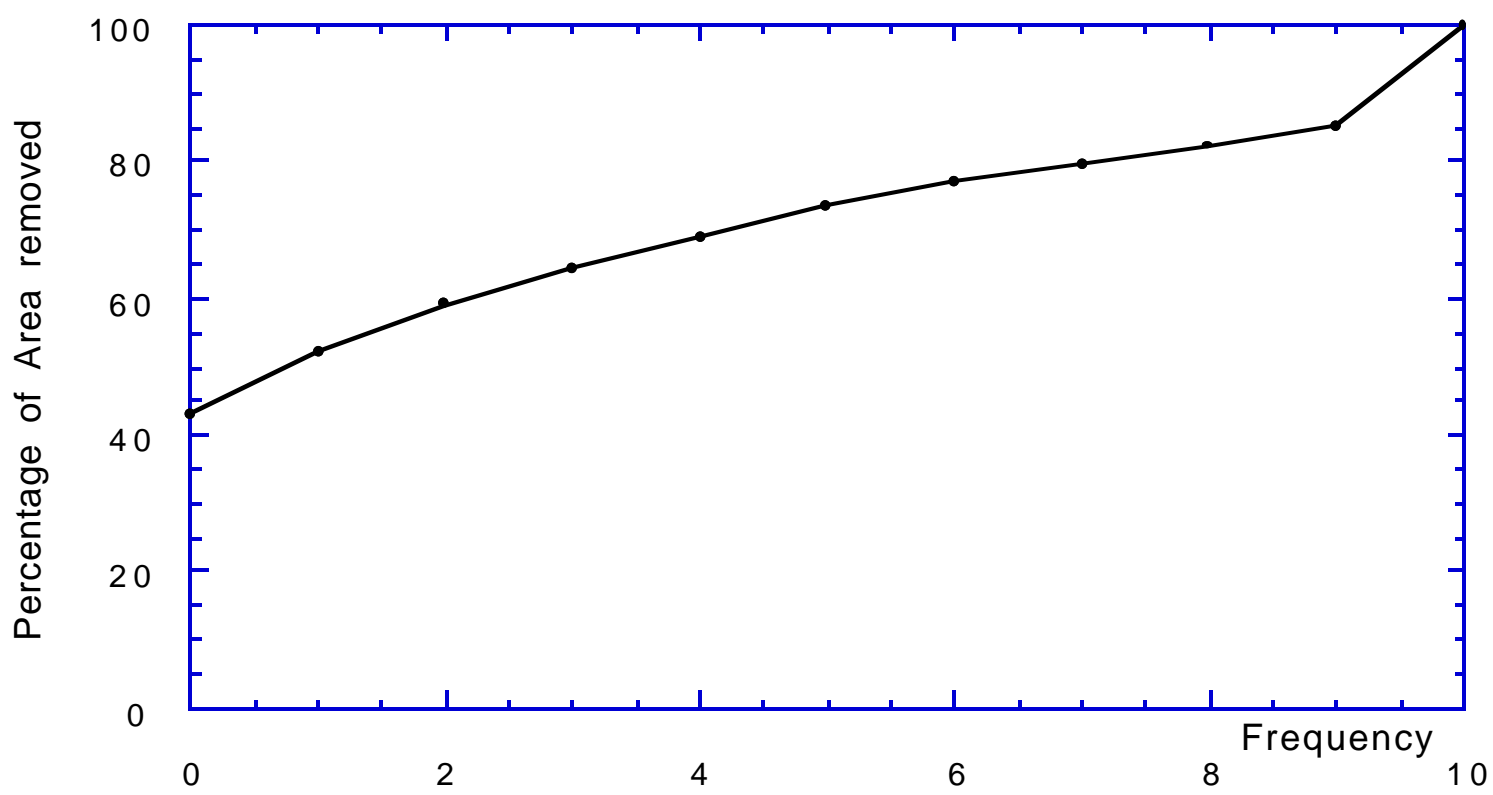

Figure 5.7b: Plot of the percentage of Cumulative area removed Vs. the frequency (number of cycles) of removal, for $\mathrm{T}=0.5$ and maximum pressure $P_{\max }=P_{\frac{1}{2}}-0.02$.

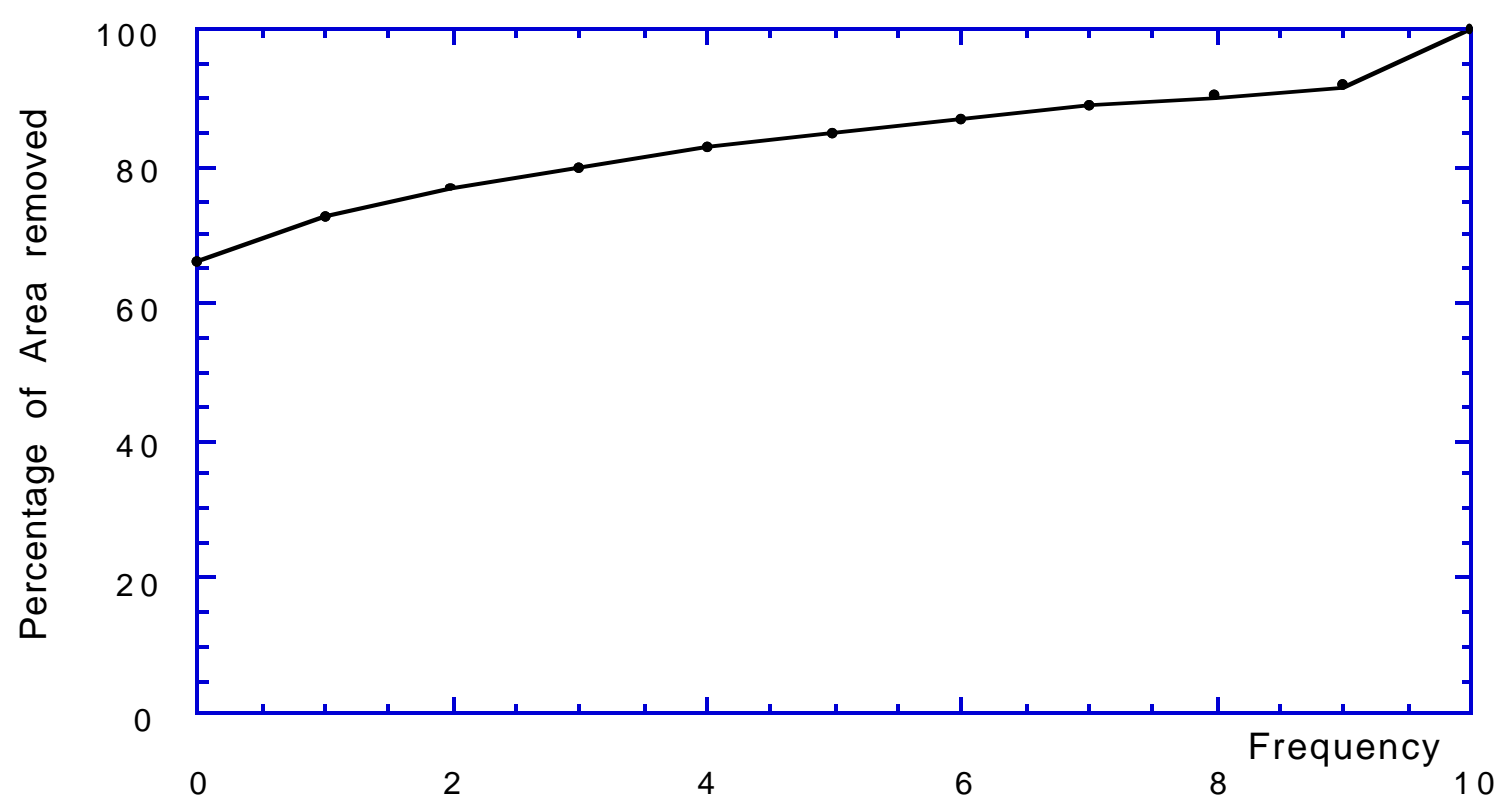

Figure 5.7c: Plot of the percentage of Cumulative area removed Vs. the frequency (number of cycles) of removal, for $\mathrm{T}=0.5$ and maximum pressure $P_{\max }=P_{\frac{1}{2}}-0.04$. 


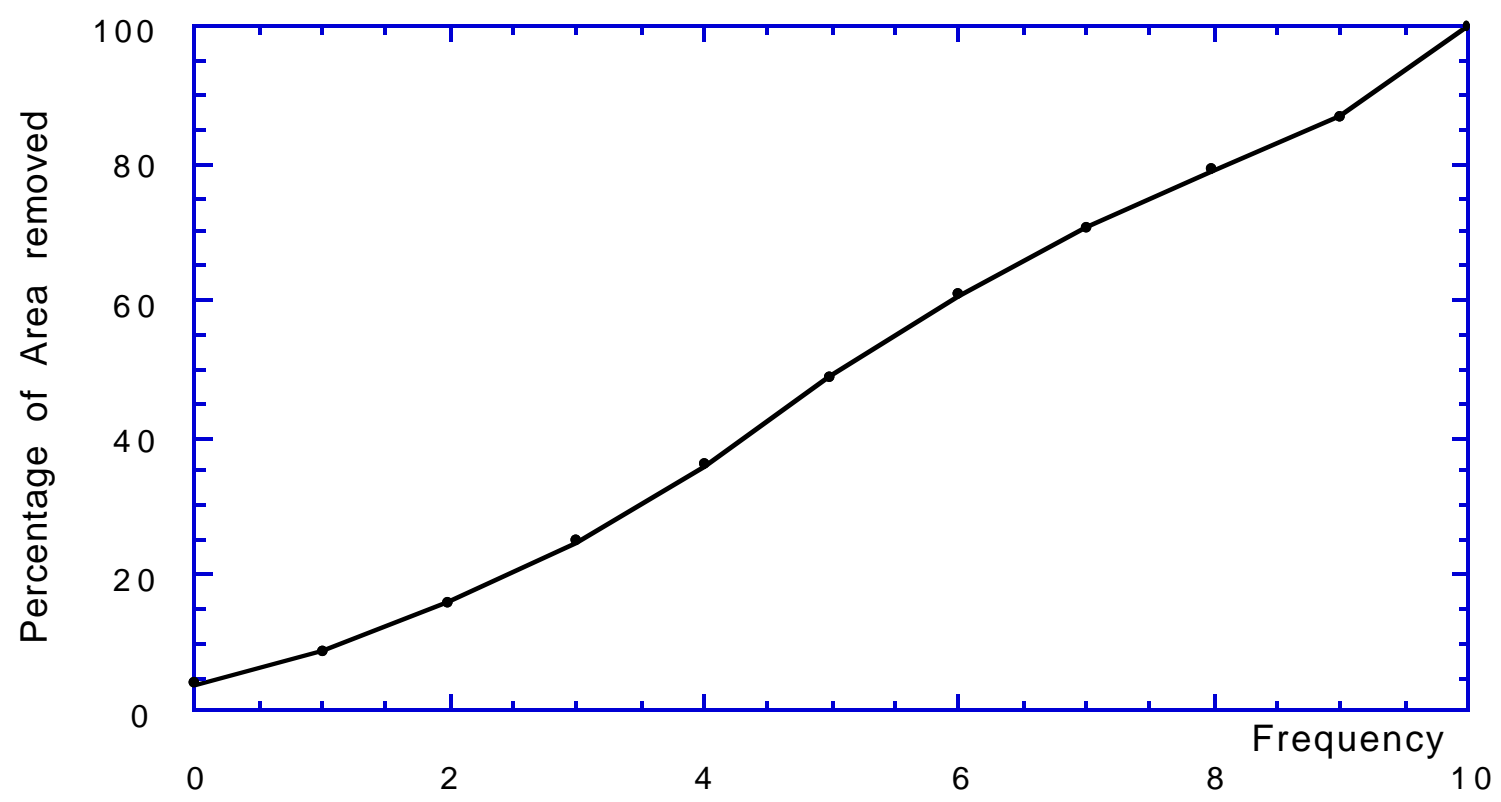

Figure 5.8a: Plot of the percentage of Cumulative area removed Vs. the frequency (number of cycles) of removal, for $\mathrm{T}=1.0$ and maximum pressure $P_{\max }=P_{\frac{1}{2}}+0.02$.

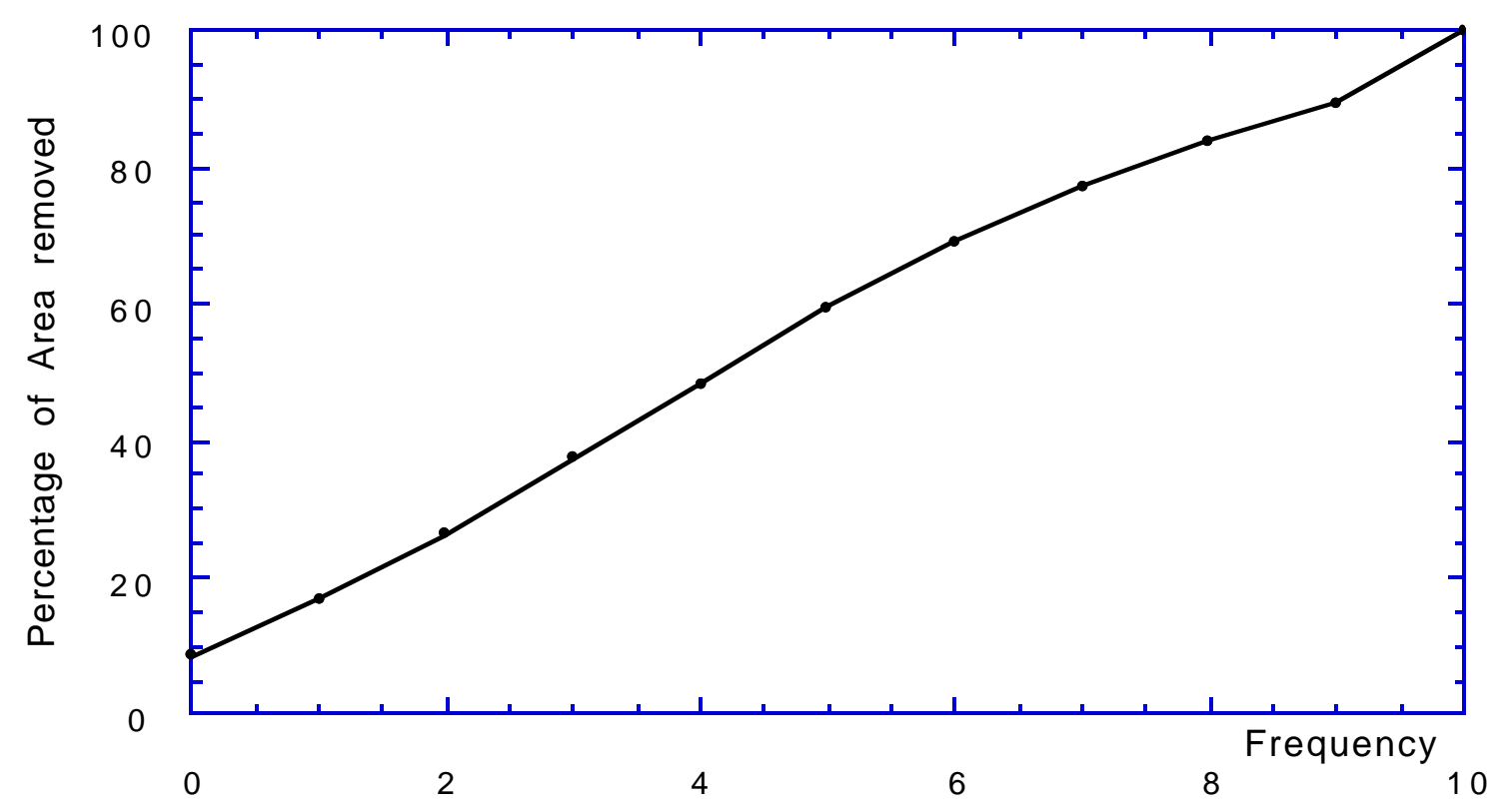

Figure 5.8b: Plot of the percentage of Cumulative area removed Vs. the frequency (number of cycles) of removal, for $\mathrm{T}=1.0$ and maximum pressure $P_{\max }=P_{\frac{1}{2}}+0.01$. 


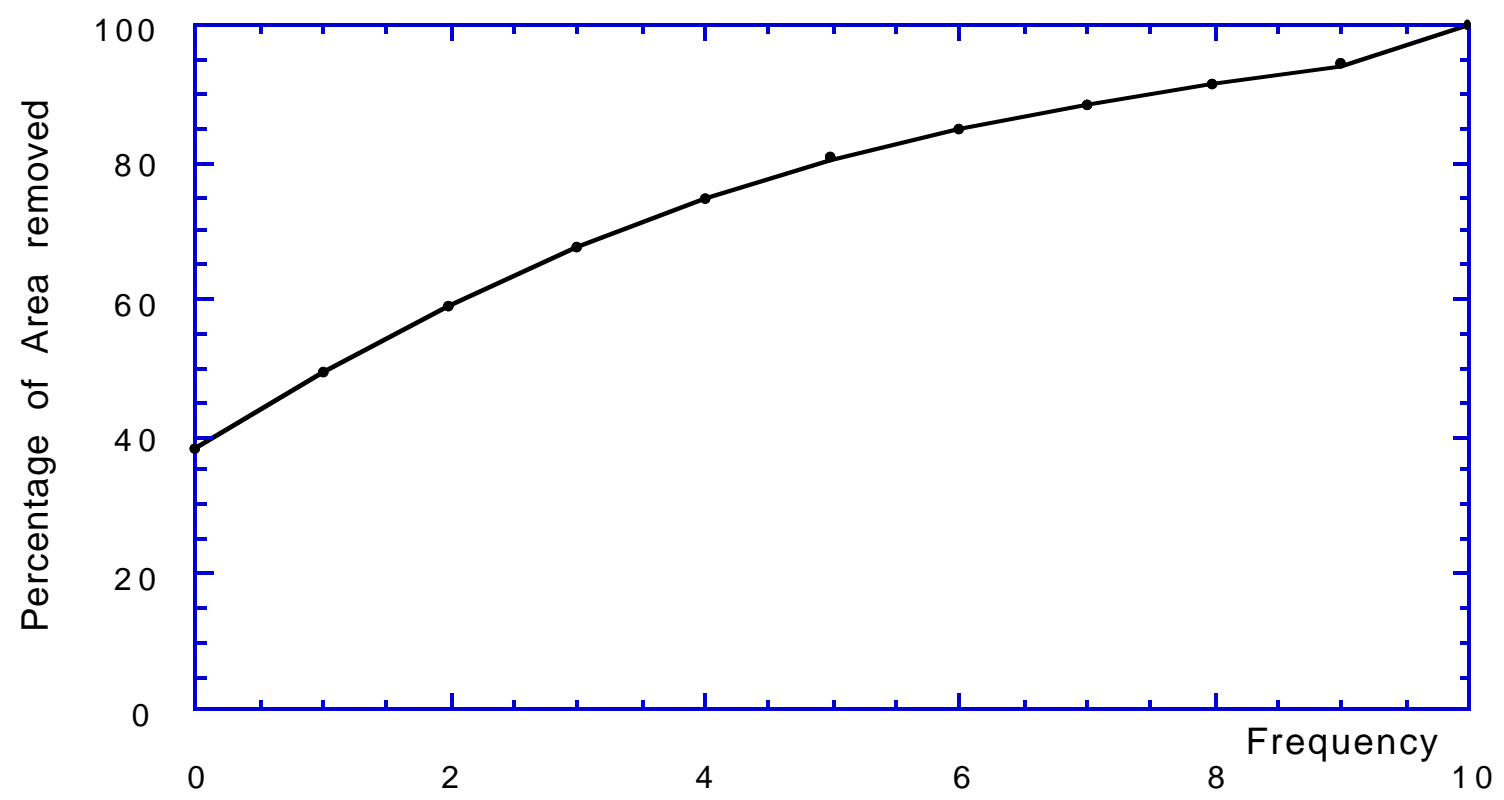

Figure 5.8c: Plot of the percentage of Cumulative area removed Vs. the frequency (number of cycles) of removal, for $\mathrm{T}=1.0$ and maximum pressure $P_{\max }=P_{\frac{1}{2}}-0.01$.

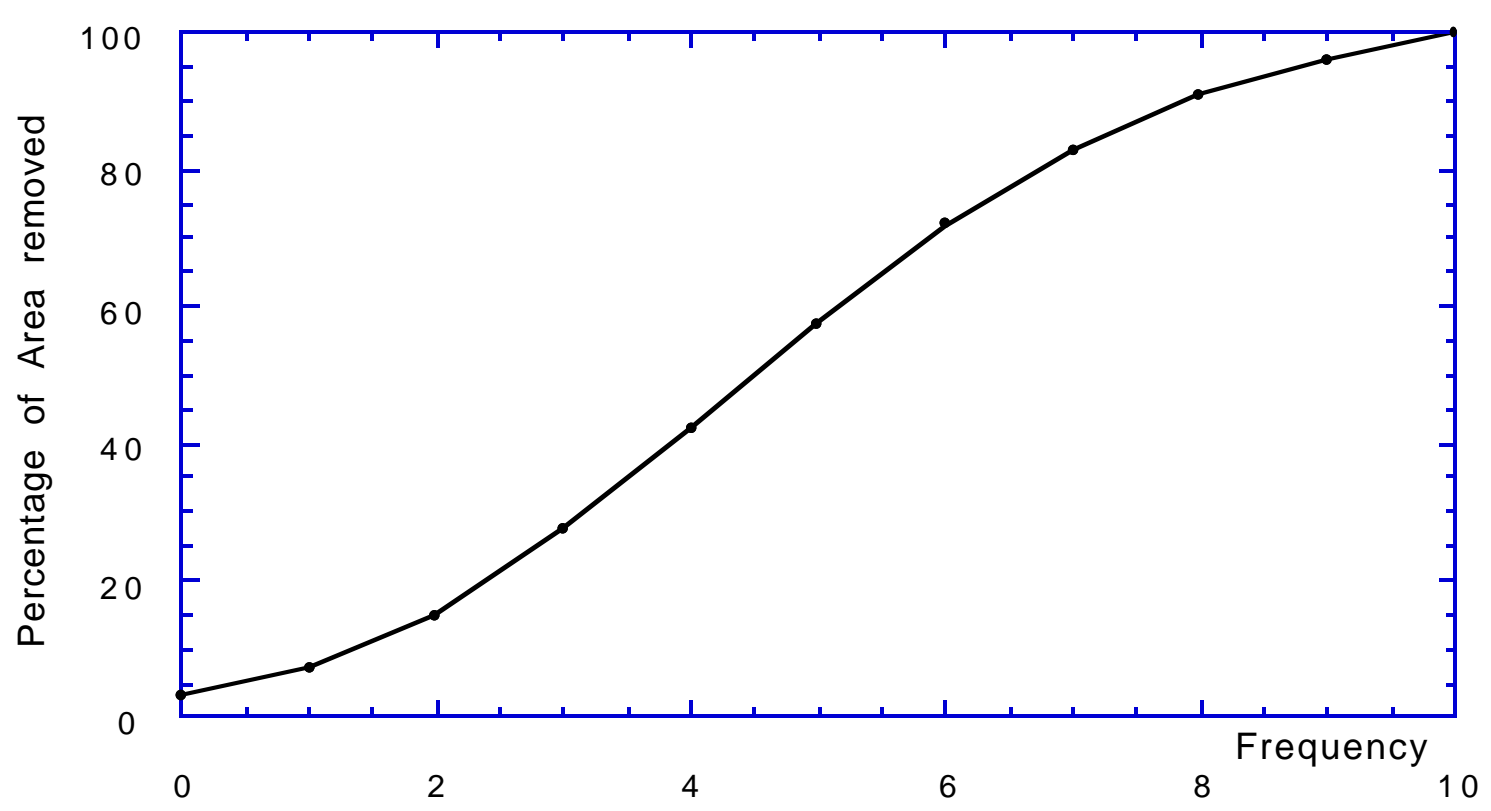

Figure 5.9a: Plot of the percentage of Cumulative area removed Vs. the frequency (number of cycles) of removal, for $\mathrm{T}=1.5$ and maximum pressure $P_{\max }=P_{\frac{1}{2}}+0.03$. 


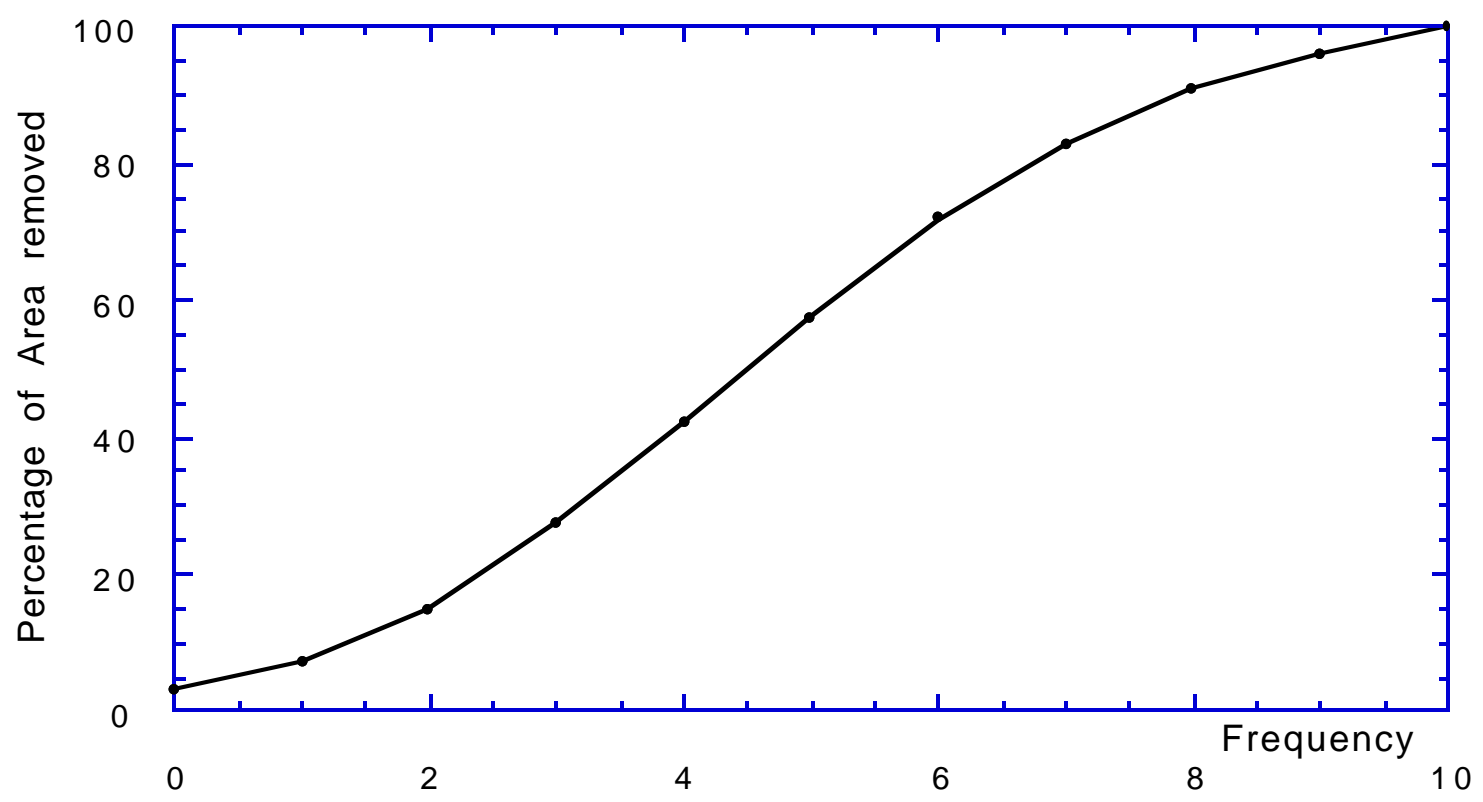

Figure 5.9b: Plot of the percentage of Cumulative area removed Vs. the frequency (number of cycles) of removal, for $\mathrm{T}=1.5$ and maximum pressure $P_{\max }=P_{\frac{1}{2}}+0.02$.

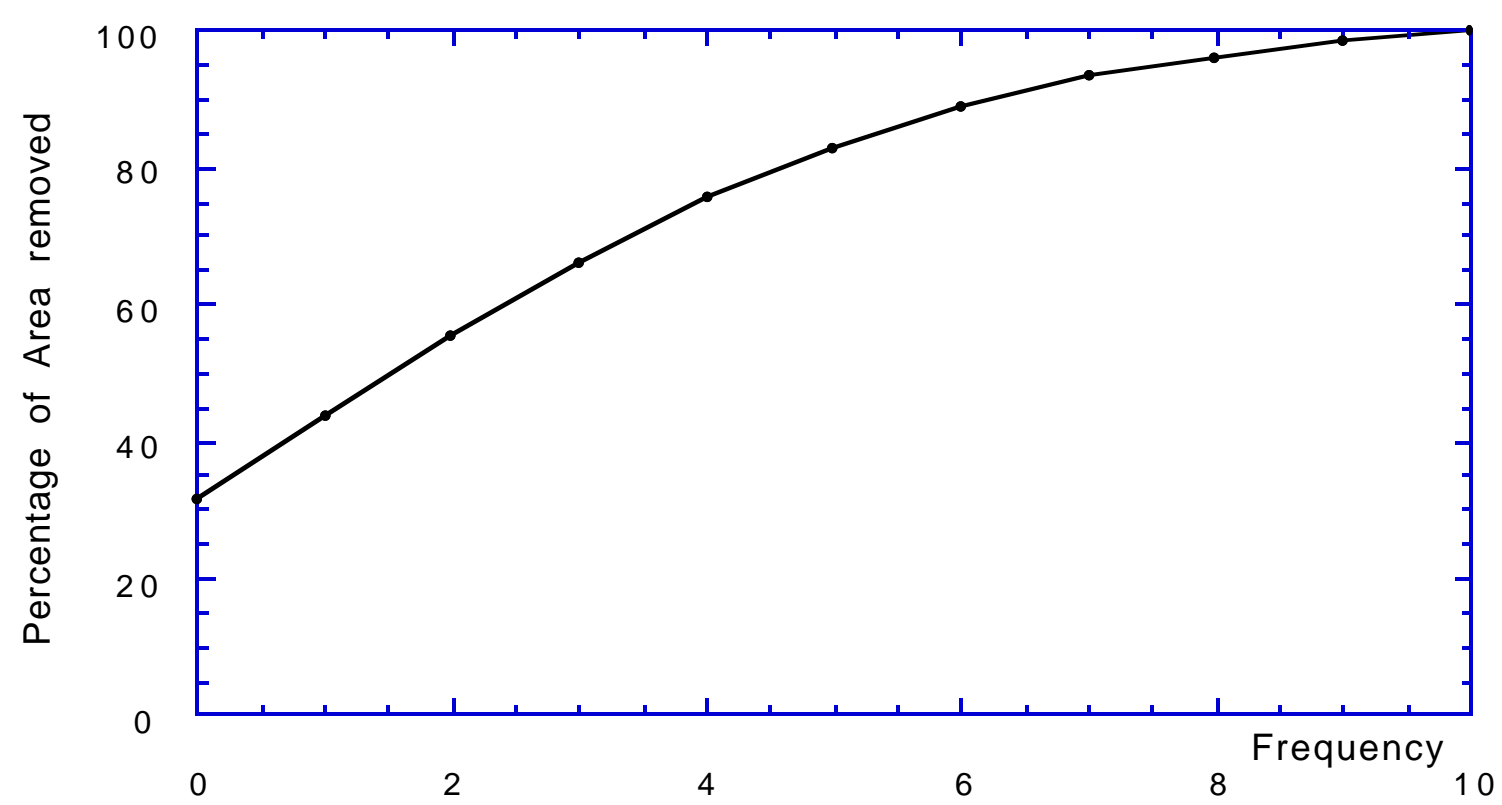

Figure 5.9c: Plot of the percentage of Cumulative area removed Vs. the frequency (number of cycles) of removal, for $\mathrm{T}=1.5$ and maximum pressure $P_{\max }=P_{\frac{1}{2}}+0.0$. 


\subsection{Discussion of the Cumulative Plots}

The plots in Figures $5.7(\mathrm{a}-\mathrm{c}), 5.8(\mathrm{a}-\mathrm{c})$ and 5.9 (a-c) can be considered as the integrals of the corresponding graphs in Figures 5.4 (a-c), $5.5(\mathrm{a}-\mathrm{c})$ and $5.6(\mathrm{a}-\mathrm{c})$. The humps (points where the derivative changes sign) in Figures 5.5a, 5.5b, 5.6a and 5.6b thus correspond to the points of inflection in Figures 5.8a, 5.8b, 5.9a and 5.9b respectively. A comparison of the trends for a particular thickness parameter $\mathrm{T}$ shows that as the applied pressure increases, the percentage of layer area that is never removed decreases, while the percentage of layer area that is removed all 10 times increases, which is as expected.

For thickness parameter $\mathrm{T}=1.0$ and maximum pressure $P_{\max }=P_{\frac{1}{2}}+0.02$ the fraction of layer mass removed is close to $60 \%$. The cumulative plot for this case has been compared with the cumulative plot that was graphed using experimental results for $60 \%$ layer removal. The two plots have been found to be in close agreement with each other with a maximum deviation of less that $10 \%$ near the midrange frequencies. This difference in the regeneration frequencies may be accounted for by variations in dust cake height, during the regeneration of the filter cake between successive cycles, whose effect has not been included in the present simulations of the fine-scale filter cake model. 


\section{Chapter Six}

\section{Conclusions}

\subsection{Conclusions}

This Chapter presents the results from the fine-scale modeling of the removal of a layer of filter cake by a time dependent pressure pulse. The use of a pressure pulse which decreases with time as the filter cake is removed, simulates the actual removal process closely. This is because a partial removal of the filter cake at each time step, increases the permeability of the filter requiring a reduced pressure drop to maintain constant flow. The simulations studied the cleaning through 10 consecutive cleaning cycles, for three filter cakes, which differed from each other in the relative strengths of their cohesive and adhesive forces. The following is a summary of the results of the study.

Cohesive Effects: The results of the simulations show that cohesive forces play a very important role in filter cake removal. Strong cohesive forces introduce cascade effects which significantly lower the pressure required to remove a given fraction of the filter cake layer. Cooperative effects introduced by these forces also cause a thicker (more cohesive) cake to be removed more efficiently.

Adhesive Effects: In order to achieve significant cleaning a higher pressure pulse was required. This high pressure caused damage (broken adhesive bonds) in the uncleaned regions. The damage was not fully repaired in the next filtration cycle, affecting the cleaning in the future cycles. Thus for strongly cohesive cakes, when the cleaning was undertaken over several (10) cycles, the adhesive forces caused a significant number of blocks to be removed in every other cycle. 
The Fine-Scale filter cake modeling has furthered an understanding of the roles which cohesive and adhesive forces play when the cleaning is conducted over several cycles. This is crucial to the development of a more efficient cleaning process.

\subsection{Future Considerations}

In the present simulations the filter cake was regenerated to the 'same height' in those areas where portions of it had been cleaned in the previous cycle. In reality this is not completely true. At the end of each cleaning cycle, when some more gas is filtered through, the previously cleaned areas fill up to a smaller height, while the uncleaned areas accumulate dust and increase in height. The filter surface now has places where the filter cake is not so thick and places where the filter cake is thicker. The effects of cohesive forces would therefore vary from region to region depending on the thickness of the deposited cake. A more realistic model would therefore be one, that take into account regional variations in the cohesive strength of the filter cake layer. 


\section{References}

[1] M. Ferer and D. H. Smith, Journal of Applied Physics 81, 1737 (1997).

[2] M. Ferer and D. H. Smith, Physical Review E 57, 866 (1998).

[3] M. Ferer and D. H. Smith, Aerosol. Sci. Technol. 29, 246 (1998).

[4] M. Ferer and D. H. Smith, Physical Review E 58, 7071 (1998).

[5] V. Z. Parton, Fracture Mechanics: From Theory to Practice (Gordon and Breach, Philadelphia, 1992).

[6] H. J. Hermann and S. Roux, Statistical Models for the Fracture of Disordered Media (North-Holland, Amsterdam, 1990).

[7] L. Golubovic, A. Peredera, Phys. Rev. E 51, 2799 (1995).

[8] H. Furukawa, Phys. Rev. E 52, 5124 (1995).

[9] J. M. Weeldon, Experimental Results from the Grimethorpe PFBC Facility, in Proceedings of the Eighth International Conference of Fluidized Bed Combustion (U. S. D. O. E./M. E. T. C., Morgantown, WV, 1985), Vol. 1.

[10] S. Berbner and F. Loffler, Pulse Jet Cleaning of Ceramic Filters, in Proceedings of the Filtech Conference-Karlsruhe, Germany, 1993 (unpublished).

[11] S. Laux, B. Giernoth, H. Bulak and U. Renz, Aspects of Pulse -Jet Cleaning of Ceramic Filter Elements, in Proceedings of the International Conference on Fluidized Bed Combustion-San Diego, CA, 1993.

[12] S. Berbner and F. Loffler, in Pulse Jet Cleaning of Rigid Ceramic Barrier Filters: Separating Hard and Brown Coal Fly Ashes at High Temperatures, in Proceedings of the Eleventh Annual Pittsburgh Coal Conference (University of Pittsburgh, Pittsburgh, PA, 1994), pp. 1357-1363.

[13] K. Morris, R. W. K. Allen and R. Clift, Filtration and Separation 24, 41 (1987).

[14] D. Koch, W. Cheung, J. P. K. Seville and R. Clift, Filtration and Separation 29, 337 (1992). 
[15] S. Berbner and F. Loffler, Powder Technology 78, 273 (1994).

[16] M. Ferer and D. H. Smith, Chemical Engineering Sci. (accepted for publication).

[17] J. Sievert and F. Loeffler, Filtration and Separation 24, 424 (1987).

[18] J. P. K. Seville, W. Cheung and R. Clift, Filtration and Separation 26, 187 (1989). 


\section{Pulkit Mathur}

\section{Education:}

08/98-05/00

Master of Science in Physics, West Virginia University, Morgantown, WV.

Thesis: Fine-Scale modeling of Failure in an Adhesive Layer.

08/93-08/96

Bachelor of Science in Physics, St. Xavier's College, Mumbai, India. 